\title{
On finite symmetries and their gauging in two dimensions
}

\author{
Lakshya Bhardwaj $^{a}$ and Yuji Tachikawa ${ }^{b}$ \\ ${ }^{a}$ Perimeter Institute for Theoretical Physics, \\ Waterloo, Ontario, N2L $2 Y 5$ Canada \\ ${ }^{b}$ Kavli Institute for the Physics and Mathematics of the Universe, University of Tokyo, \\ Kashiwa, Chiba 277-8583, Japan \\ E-mail: lbhardwaj@pitp.ca, yuji.tachikawa@ipmu.jp
}

ABSTRACT: It is well-known that if we gauge a $\mathbb{Z}_{n}$ symmetry in two dimensions, a dual $\mathbb{Z}_{n}$ symmetry appears, such that re-gauging this dual $\mathbb{Z}_{n}$ symmetry leads back to the original theory. We describe how this can be generalized to non-Abelian groups, by enlarging the concept of symmetries from those defined by groups to those defined by unitary fusion categories. We will see that this generalization is also useful when studying what happens when a non-anomalous subgroup of an anomalous finite group is gauged: for example, the gauged theory can have non-Abelian group symmetry even when the original symmetry is an Abelian group. We then discuss the axiomatization of two-dimensional topological quantum field theories whose symmetry is given by a category. We see explicitly that the gauged version is a topological quantum field theory with a new symmetry given by a dual category.

KeYwords: Anyons, Discrete Symmetries, Global Symmetries, Topological Field Theories

ARXIV EPRINT: 1704.02330 


\section{Contents}

1 Introduction 1

2 Re-gauging of finite group gauge theories 3

2.1 Abelian case 3

2.2 Non-Abelian case 4

3 Symmetries as categories in two dimensions $\quad 6$

$\begin{array}{ll}3.1 \text { Basic notions of symmetry categories } & 6\end{array}$

$\begin{array}{lll}3.2 \text { Comments } & 14\end{array}$

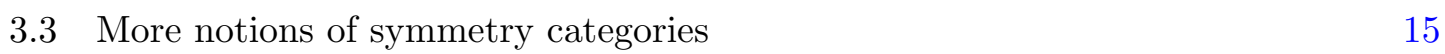

$\begin{array}{lll}3.4 & \text { Groups and representations of groups as symmetry categories } & 16\end{array}$

4 Gaugings and symmetry categories $\quad 19$

$\begin{array}{ll}4.1 & \text { Module and bimodule categories }\end{array}$

4.2 Duality of $\mathcal{C}(G)$ and $\operatorname{Rep}(G) \quad 21$

4.3 Gauging by an algebra object 22

4.4 Symmetries of the gauged theory from bimodules for the algebra object 24

4.5 Gauging of $\mathcal{C}(G)$ to get $\operatorname{Rep}(G)$ and vice versa 28

$\begin{array}{ll}4.6 \text { Gaugings and module categories } & 29\end{array}$

4.7 (Re-)gauging and its effect on the symmetry category 31

4.8 The effect of the gauging on Hilbert space on $S^{1} 32$

5 More examples of symmetry categories and their gauging 35

5.1 Symmetry category with two simple lines 36

5.2 Symmetry category of SU(2) WZW models and other RCFTs 36

5.3 Gauging a subgroup of a possibly-anomalous group 37

5.4 Integral symmetry categories of total dimension $6 \quad 40$

5.5 Integral symmetry categories of total dimension $8 \quad 41$

$\begin{array}{lll}5.6 & \text { Tambara-Yamagami categories } & 42\end{array}$

$6 \quad$ 2d TFT with $\mathcal{C}$ symmetry and their gauging $\quad 45$

$\begin{array}{lll}6.12 \mathrm{~d} \text { TFTs without symmetry } & 45\end{array}$

$\begin{array}{lll}\text { 6.2 } & \text { TFT with } \mathcal{C} \text { symmetry on a cylinder } & 48\end{array}$

6.3 TFT with $\mathcal{C}$ symmetry on a general geometry $\quad 52$

$\begin{array}{ll}6.4 \text { Gauged TFT with the dual symmetry } & 58\end{array}$

$\begin{array}{lll}7 & \text { Conclusions } & 60\end{array}$

$\begin{array}{ll}\text { A Group cohomology } & 62\end{array}$ 


\section{Introduction}

Let us start by considering a two-dimensional theory $T$ with $\mathbb{Z}_{n}$ symmetry. We can gauge it to get the gauged theory $T / \mathbb{Z}_{n}$. This gauged theory is known to have a new $\mathbb{Z}_{n}$ symmetry, and re-gauging it gives us back the original theory: $T / \mathbb{Z}_{n} / \mathbb{Z}_{n}=T$ [1]. It is also well-known that this phenomenon generalizes to any arbitrary finite Abelian group $G$. That is, gauging a theory $T$ with $G$ symmetry results in a theory $T / G$ with a new finite Abelian group symmetry $\hat{G}$ such that gauging the new theory by the new symmetry takes us back to the original theory: $T / G / \hat{G}=T$. One natural question arises: can it be generalized to higher dimensions? Yes, according to [2], where the generalized concept of $p$-form symmetries was introduced. Our investigation starts from a related but different question: can it be generalized to non-Abelian finite groups?

The answer is again yes [3], and it again requires a generalization of the concept of symmetries, but in a direction different from that of [2]. To explain this, let us pose what we have said above in a different way. Traditionally, we say that $T$ has symmetry $G$ if we can find unitary operators $U_{g}$ labeled by elements $g \in G$ whose action on the Hilbert space commutes with the Hamiltonian. When $G$ is Abelian, the information about $G$ is captured in $T / G$ via unitary operators $U_{\hat{g}}$ labeled by elements $\hat{g} \in \hat{G}$ which commute with the Hamiltonian of $T / G$. When $G$ is non-Abelian, we will argue that the information of $G$ in $T / G$ is captured by operators $U_{i}$ which still commute with the Hamiltonian but these operators are now in general non-unitary. These operators can be constructed by wrapping a Wilson line for $G$ along the spatial circle. Hence, Wilson lines should be thought of as generalized symmetries for the theory $T / G$. In fact, we will also argue that there is a natural notion of gauging this symmetry formed by Wilson lines such that gauging $T / G$ results back in the original theory $T$.

This raises the following question: how do we specify a generalized symmetry that a theory can admit? In this paper, we give an answer to this question: a general finite symmetry of a two-dimensional theory is specified by a structure which is known to mathematicians in the name of unitary fusion categories. We prefer to call it symmetry categories. ${ }^{1}$ For the gauged theory $T / G$ for possibly non-Abelian group $G$, the Wilson line operators form $\operatorname{Rep}(G)$, which is a symmetry category formed by the representations of $G$. Similarly, a general symmetry category $\mathcal{C}$ physically corresponds to more general line operators of $T$.

We also discuss how a symmetry category $\mathcal{C}$ can be gauged. It turns out that there is no canonical way of gauging a generic symmetry category. Pick one way $\mathcal{M}$ of gauging the symmetry $\mathcal{C}$ of a theory $T$. Denote the gauged theory by $T / \mathcal{M}$ and its symmetry category by $\mathcal{C}^{\prime}$. It then turns out that there exists a dual way $\mathcal{M}^{\prime}$ of gauging $\mathcal{C}^{\prime}$ such that

\footnotetext{
${ }^{1}$ We do not claim that this is the ultimate concept for the 0-form finite symmetry in two dimensions; there still might be a generalization in the future. For example, in other spacetime dimensions, a proposed generalization was to use the concept of p-groups, see e.g. [4], and one might want to unify the two approaches. We also note that this extension of the concept of the symmetry in two dimensions from groups to categories was already proposed by many other authors in the past, and we are merely shedding a light to it from a slightly different direction. We will come back to this point later in the Introduction.
} 
$T / \mathcal{M} / \mathcal{M}^{\prime}$ is equivalent to $T$. This generalizes the fact that regauging the gauged theory with symmetry $\operatorname{Rep}(G)$ gives back the original theory with symmetry $G$.

This generalization of the concept of symmetry allows us not only to perform the regauging of non-Abelian gauge theories, but also to answer various other questions. First of all, we will see that symmetry categories $\mathcal{C}$ capture symmetries together with their anomalies. Then, the machinery we spell out allows us to compute what is the symmetry of the gauge theory $T / H$ when we gauge a subgroup $H$ of a possibly anomalous flavor symmetry $G$. For example, if we gauge a non-anomalous $\mathbb{Z}_{2}$ subgroup of $\mathbb{Z}_{2} \times \mathbb{Z}_{2} \times \mathbb{Z}_{2}$ with a suitable choice of the anomaly, we can get non-anomalous non-Abelian symmetry $D_{8}$ and $Q_{8}$, the dihedral group and the quaternion group of order $8 .^{2}$ In general, the symmetry $\mathcal{C}$ of the gauged theory is neither a group nor $\operatorname{Rep}(G)$ for a finite group, and we need the concept of symmetry categories to describe it.

There are also vast number of symmetry categories not related to finite groups, formed by topological line operators of two-dimensional rational conformal field theories (RCFTs). In particular, any unitary modular tensor category, or equivalently any Moore-Seiberg data, can be thought of as a symmetry category, by forgetting the braiding.

We should emphasize here that this generalization of the concept of symmetry from that defined by groups to that defined by categories was already done long ago by other authors, belonging to three somewhat independent lines of studies, namely in the study of the subfactors and operator algebraic quantum field theories, in the study of representation theory, and in the study of RCFTs. Each of the communities produced vast number of papers, and not all of them can be cited here. We recommend to the readers textbooks by Bischoff, Longo, Kawahigashi and Rehren [6] and Etingof, Gelaki, Nikshych and Ostrik [7] from the first two communities and the articles by Carqueville and Runkel [8] and by Brunner, Carqueville and Plencner [9] from the third community as the starting points.

Our first aim in this paper is then to summarize the content of these past works in a way hopefully more accessible to other researchers of quantum field theory, including the authors of this paper themselves, emphasizing the point of view related to the modern study of symmetry protected topological phases. What we explain in this first part of the paper is not new, except possibly the way of the presentation, and can all be found in the literature in a scattered form.

Our second aim is to axiomatize two-dimensional topological quantum field theories (TFTs) whose symmetry is given by a symmetry category $\mathcal{C}$. This is a generalization of the

\footnotetext{
${ }^{2}$ Recently in [5], Gaiotto, Kapustin, Komargodski and Seiberg performed an impressive study of the phase structure of thermal $4 \mathrm{~d} \mathfrak{s u}(2)$ Yang-Mills theory. One important step in the analysis is the symmetry structure of the thermal system, which is essentially three-dimensional. As a dimensional reduction from $4 \mathrm{~d}$, the system has a $\mathbb{Z}_{2} \times \mathbb{Z}_{2}$ 0-form symmetry and a $\mathbb{Z}_{2}$ 1-form symmetry, with a mixed anomaly. Then the authors gauged the $\mathbb{Z}_{2} 1$-form symmetry, and found that the total 0 -form symmetry is now $D_{8}$. This $D_{8}$ was then used very effectively to study the phase diagram, but that part of their paper does not directly concern us here. Their analysis of turning an anomalous Abelian symmetry by gauging a non-anomalous subgroup into a non-Abelian symmetry is a $3 \mathrm{~d}$ analogue of what we explain in $2 \mathrm{~d}$. See their section 4.2 , appendix B and appendix C. Clearly an important direction to pursue is to generalize their and our constructions to arbitrary combinations of possibly-higher-form symmetries in arbitrary spacetime dimensions, but that is outside of the scope of this paper.
} 
work by Moore and Segal [10], where two-dimensional TFTs with finite group symmetry were axiomatized. We write down basic sets of linear maps between (tensor products of) Hilbert spaces on $S^{1}$ and basic consistency relations among them which guarantee that a unique linear map is associated to any surface with $m$ incoming circles and $n$ outgoing circles together with arbitrary network of line operators from $\mathcal{C}$.

The rest of the paper is organized as follows. First in section 2, as a preliminary, we recall how gauging of a finite Abelian symmetry $G$ can be undone by gauging the new finite Abelian symmetry $\hat{G}$, and then briefly discuss how this can be generalized to non-Abelian symmetries $G$, by regarding $\operatorname{Rep}(G)$ as a symmetry. This effort of generalizing the story to a non-Abelian group makes the possibility and the necessity of a further generalization to symmetry categories manifest. We exploit this possibility and describe the generalization in detail in subsequent sections.

Second, we have two sections that form the core of the paper. In section 3, we introduce the notion of symmetry categories, and discuss how we can regard as symmetry categories both a finite group $G$ with an anomaly $\alpha$ and the collection $\operatorname{Rep}(G)$ of representations of $G$. We then explain in section 4 that physically distinct gaugings of a given symmetry category $\mathcal{C}$ correspond to indecomposable module categories $\mathcal{M}$ of $\mathcal{C}$, and we describe how to obtain the new symmetry $\mathcal{C}^{\prime}$ of the theory $T / \mathcal{M}$ for a given theory $T$ with a symmetry $\mathcal{C}$.

Third, in section 5 , we give various examples illustrating the notions introduced up to this point. Examples include the form of new symmetry categories $\mathcal{C}^{\prime}$ when we gauge a non-anomalous subgroup $H$ of an anomalous finite group $G$, and the symmetry categories of RCFTs.

Fourth, in section 6 , we move on to the discussion of the axioms of two-dimensional TFTs whose symmetry is given by a symmetry category $\mathcal{C}$. We also construct the gauged TFTs $T / \mathcal{M}$ given an original TFT $T$ with a symmetry category $\mathcal{C}$ and a gauging specified by its module category $\mathcal{M}$. Sections 5 and 6 can be read independently.

Finally, we conclude with a brief discussion of what remains to be done in section 7 . We have an appendix A where we review basic notions of group cohomology used in the paper.

Before proceeding, we note that we assume that the space-time is oriented throughout the paper. We also emphasize that all the arguments we give, except in section 6 , apply to non-topological non-conformal $2 \mathrm{~d}$ theories.

\section{Re-gauging of finite group gauge theories}

\subsection{Abelian case}

Let us start by reminding ourselves the following well-known fact [1]:

Let $T$ be a $2 d$ theory with flavor symmetry given by an Abelian group $G$. Let us assume that $G$ is non-anomalous and can be gauged, and denote the resulting theory by $T / G$. Then this theory has the flavor symmetry $\hat{G}$, which is the Pontrjagin dual of $G$, such that $T / G / \hat{G}=T$.

Recall the definition of the Pontrjagin dual $\hat{G}$ of an Abelian group $G$. As a set, it is given by

$$
\hat{G}=\{\chi: G \rightarrow \mathrm{U}(1) \mid \chi \text { is an irreducible representation }\} .
$$


Note that $\chi$ is automatically one-dimensional. Therefore the product of two irreducible representations is again an irreducible representation, which makes $\hat{G}$ into a group. $G$ and $\hat{G}$ are isomorphic as a group but it is useful to keep the distinction because there is no canonical isomorphism between them.

In the literature on $2 \mathrm{~d}$ theories, gauging of a finite group $G$ theory is more commonly called as orbifolding by $G$, and the fact above is often stated as follows: a $G$-orbifold has a dual $\hat{G}$ symmetry assigning charges to twisted sectors, and orbifolding again by this dual $\hat{G}$ symmetry we get the original theory back. This dual $\hat{G}$ symmetry is also known as the quantum symmetry in the literature.

This fact can be easily shown as follows. Let $Z_{T}[M, A]$ denote the partition function of $T$ on $M$ with the external background $G$ gauge field $A$. Here $A$ can be thought of as taking values in $H^{1}(M, G)$. Then the partition function of the gauged theory $T / G$ on $M$ is given by $Z_{T / G}[M] \propto \sum_{A} Z_{T}[M, A]$. Here and in the following we would be cavalier on the overall normalization of the partition functions. More generally, with the background gauge field $B$ for the dual $\hat{G}$ symmetry, the partition function is given by

$$
Z_{T / G}[M, B] \propto \sum_{A} e^{i(B, A)} Z_{T}[M, A]
$$

where $B \in H^{1}(M, \hat{G})$ and $e^{i(B, A)}$ is obtained by the intersection pairing

$$
e^{i(-,-)}: H^{1}(M, \hat{G}) \times H^{1}(M, G) \rightarrow H^{2}(M, \mathrm{U}(1)) \simeq \mathrm{U}(1) .
$$

The equation (2.2) says that the partition function of $T / G$ is essentially the discrete Fourier transform of that of $T$, and therefore we dually have $T=T / G / \hat{G}$ :

$$
Z_{T}[M, A] \propto \sum_{B} e^{i(A, B)} Z_{T / G}[M, B]
$$

This statement was generalized to higher dimensions in e.g. [2]:

Let $T$ be a d-dimensional theory with p-form flavor symmetry given by an Abelian group $G$. Let us assume that $G$ is non-anomalous and can be gauged, and denote the resulting theory by $T / G$. Then this theory has the dual $(d-2-p)$ form flavor symmetry $\hat{G}$, such that $T / G / \hat{G}=T$.

The derivation is entirely analogous to the 2 d case, except that now $A \in H^{p+1}(M, G)$ and $B \in H^{d-1-p}(M, \hat{G})$.

\subsection{Non-Abelian case}

The facts reviewed above means that the finite Abelian gauge theory $T / G$ still has the full information of the original theory $T$, which can be extracted by gauging the dual symmetry $\hat{G}$. It is natural to ask if this is also possible when we have a non-Abelian symmetry $G$, which we assume to be an ordinary 0 -form symmetry. 
This is indeed possible ${ }^{3}$ by suitably restating the derivation above, but we will see that we need to extend the concept of what we mean by symmetry. To show this, we first massage (2.4) in a suitable form which admits a straightforward generalization. Let us consider the case of $Z_{T}[M, A=0]$ for illustration. By Poincare duality, $B$ can also be represented as an element of $H_{1}(M, \hat{G})$. Then, (2.4) can be rewritten as

$$
Z_{T}[M] \propto \sum_{\hat{g}_{1}, \cdots, \hat{g}_{n}} Z_{T / G}\left[M, \hat{g}_{1}, \cdots, \hat{g}_{n}\right]
$$

where $i \in\{1, \cdots, n\}$ labels generators of $H_{1}(M)$ and $\hat{g}_{i}$ is an element of $\hat{G}$ associated to the cycle labeled by $i$. Each summand on the right hand side, $Z_{T / G}\left[M, \hat{g}_{1}, \cdots, \hat{g}_{n}\right]$, is then the expectation value of Wilson loops in representations labeled by $\hat{g}_{i}$ placed along the cycle $i$.

Now, we can sum the $\hat{G}$ elements for each $i$ separately to obtain

$$
Z_{T}[M] \propto Z_{T / G}\left[M, W_{1}^{\mathrm{reg}}, \cdots, W_{n}^{\mathrm{reg}}\right]
$$

where $W_{i}^{\text {reg }}$ denotes the insertion of a Wilson line in the regular representation along the cycle $i$. This is because, for an abelian $G$, the regular representation is just the sum of representations corresponding to elements $\hat{g}$ of $\hat{G}$.

The relation (2.6) says that by inserting all possible Wilson lines on all possible cycles, we are putting the delta function for the original gauge field $A$. We now note that the relation (2.6) holds for a non-Abelian $G$ as well, if we insert $W_{i}^{\text {reg }}$ not only for the generators of $H_{1}(M)$ but for the generators of $\pi_{1}(M)$. This can be seen by the fact that $\operatorname{tr} g$ in the regular representation is nonzero if and only if $g$ is the identity.

The identity (2.6) means that the ungauged theory $T$ can be recovered from the gauged theory $T / G$ by inserting line operators $W_{i}^{\text {reg }}$ in an appropriate manner. This is analogous to the construction of the gauged theory $T$ from the ungauged theory $T$ by inserting line operators representing the $G$ symmetry in an appropriate manner. Given the importance of Wilson lines in recovering the information of the ungauged theory, we assign the status of dual symmetry to Wilson lines.

Let us phrase it another way. When $G$ is Abelian, the dual $(d-2)$-form $\hat{G}$ symmetries can be represented by 1-cycles labeled by elements of $\hat{G}$, forming a group. When $G$ is nonAbelian, the dual symmetry can still be represented by 1-cycles labeled by representations $\operatorname{Rep}(G)$ of $G$. We can still multiply lines, corresponding to the tensor product of the representations, and this operation reduces to group multiplication of $\hat{G}$ in the abelian case. But $\operatorname{Rep}(G)$ is not a group if $G$ is non-Abelian. Therefore this is not a flavor symmetry group, it is rather a flavor symmetry something. We summarize this observation as follows:

Let $T$ be a d-dimensional theory with 0-form flavor symmetry given by a possibly non-Abelian group $G$. Let us assume that $G$ is non-anomalous and can be gauged, and denote the resulting theory by $T / G$. Then this theory has $\operatorname{Rep}(G)$ as the dual $(d-2)$-form flavor symmetry 'something', such that $T / G / \operatorname{Rep}(G)=T$.

\footnotetext{
${ }^{3}$ That this is possible was already shown for two-dimensional theories in [3], as an example of a much more general story, which we will also review in the forthcoming sections. Here we describe the construction in an elementary language.
} 


\section{Symmetries as categories in two dimensions}

Any finite group, possibly non-Abelian, can be the 0-form symmetry group of a theory. In addition to this, we saw in the last section that $\operatorname{Rep}(G)$, the representations of $G$, can also be the $(d-2)$-form symmetry something of a $d$-dimensional theory. We do not yet have a general understanding of what should be this something in general $d$ dimensions for general combinations of various $p$-form symmetries. However, at least for $d=2$ and $p=0$, we already have a clear concept for this something in the literature, which includes both groups $G$ and representations of groups $\operatorname{Rep}(G)$ and much more. In this section we explain what it is.

\subsection{Basic notions of symmetry categories}

In two dimensions, a 0 -form finite symmetry element can be represented by a line operator with a label $a$. Inserting this line operator on a space-like slice $S$ corresponds to acting on the Hilbert space associated to $S$ by a possibly non-unitary operator $U_{a}$ corresponding to the symmetry element. Moreover, $U_{a}$ commutes with the Hamiltonian $H$ associated to any foliation of the two-dimensional manifold. In addition, $U_{a}$ cannot change under a continuous deformation of its path. Therefore the line operator under consideration is automatically topological.

Topological line operators, in general, form a structure which mathematicians call a tensor category. We want to restrict our attention to topological line operators describing a finite symmetry. Such topological line operators form a structure which mathematicians call a unitary fusion category. We call it instead as a symmetry category, to emphasize its role as the finite symmetry of unitary two-dimensional quantum field theory. We start by stating the slogan, and then fill in the details:

Finite flavor symmetries of $2 d$ theories are characterized by symmetry categories.

\subsubsection{Objects}

The objects in a symmetry category $\mathcal{C}$ correspond to topological line operators generating the symmetry. More precisely, a theory $T$ admitting the symmetry $\mathcal{C}$ will admit topological line operators labeled by the objects of $\mathcal{C}$. Henceforth, we will drop the adjective topological in front of line operators.

For any line operator labeled by an object $a$, we have a partition function of $T$ with the line inserted along an oriented path $C$, which we can denote as

$$
\langle\cdots a(C) \cdots\rangle
$$

where the dots stand for additional operators inserted away from $C$.

\subsubsection{Morphisms}

The morphisms in a symmetry category $\mathcal{C}$ correspond to topological local point operators which can be inserted between two lines. More precisely, consider two labels $a$ and $b$, and a path $C$ such that up to a point $p \in C$ we have the label $a$ and from the point $p$ we have 


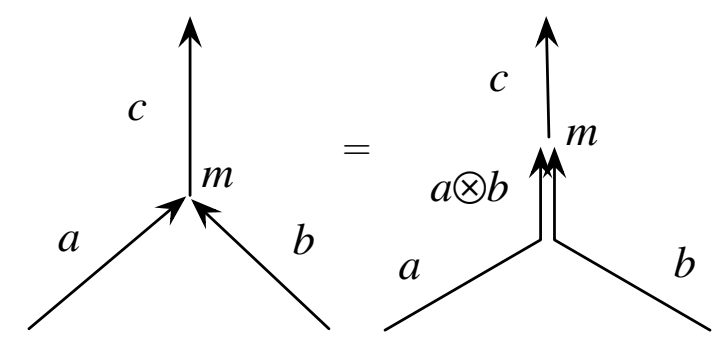

Figure 1. Two lines with the labels $a$ and $b$ can be fused to form a line with the label $a \otimes b$.

the label $b$. Then, we can insert a possibly-line-changing topological operator labeled by $m$ at $p$. We call such $m$ a morphism from $a$ to $b$, and denote this statement interchangeably as either

$$
m: a \rightarrow b \quad \text { or } \quad m \in \operatorname{Hom}(a, b) .
$$

The set $\operatorname{Hom}(a, b)$ is taken to be a complex vector space and it labels (a subspace of) topological local operators between line operators corresponding to $a$ and $b$ in $T$. From now on, we would drop the adjective topological in front of local operators.

\subsubsection{Existence of a trivial line}

$\mathcal{C}$ contains an object 1 , which labels the trivial line of $T$. We have

$$
\langle\cdots 1(C) \cdots\rangle=\langle\cdots \cdots\rangle .
$$

\subsubsection{Additive structure}

Given two objects $a$ and $b$, there is a new object $a \oplus b$ in $\mathcal{C}$. In terms of lines in $T$, we have

$$
\langle\cdots(a \oplus b)(C) \cdots\rangle=\langle\cdots a(C) \cdots\rangle+\langle\cdots b(C) \cdots\rangle .
$$

We abbreviate $a \oplus a$ by $2 a, a \oplus a \oplus a$ by $3 a$, etc. The linear operator $U_{a \oplus b}$ acting on the Hilbert space obtained by wrapping $a \oplus b$ on a circle is then given by $U_{a}+U_{b}$.

\subsubsection{Tensor structure}

Given two objects $a$ and $b$, we have an object $a \otimes b$ in $\mathcal{C}$. This corresponds to considering two parallel-running line operators $a$ and $b$ as one line operator. The linear operator $U_{a \otimes b}$ acting on the Hilbert space obtained by wrapping $a \otimes b$ on a circle is then given by $U_{a} U_{b}$.

The trivial object 1 acts as an identity for this tensor operation. That is, there exist canonical isomorphisms $a \otimes 1 \simeq a$ and $1 \otimes a \simeq a$ for each object $a$. We can always find an equivalent category in which these isomorphisms are trivial, that is $a \otimes 1=1 \otimes a=a$. Hence, we can assume that these isomorphisms have been made trivial in $\mathcal{C}$. Henceforth, the unit object will also be referred to as the identity object.

Consider three lines $C_{1,2,3}$ meeting at a point $p$, with $C_{1,2}$ incoming and $C_{3}$ outgoing. We can put the label $a, b, c$ on $C_{1,2,3}$, respectively. We demand that the operators we can put at the junction point $p$ is given by $m \in \operatorname{Hom}(a \otimes b, c)$. This label $a \otimes b$ corresponds to a composite line as can be seen by the following topological deformation shown in figure 1 . 
The definition of $a \otimes b$ here includes a choice of the implicit junction operator where the lines labeled by $a, b$ and $a \otimes b$ meet. In this paper, whenever we draw figures with such implicit junction operators, we always choose the operator to be the one labeled by the identity morphism id $: a \otimes b \rightarrow a \otimes b$.

\subsubsection{Simplicity of the identity, semisimplicity, and finiteness}

The simple objects $a \in \mathcal{C}$ are objects for which $\operatorname{Hom}(a, a)$ is one-dimensional. In general, for any object $x$, there is always a canonical identity morphism from $x$ to $x$ which labels the identity operator on the line labeled by $x$. For a simple object $a$, the existence of the identity morphism implies that there is a natural isomorphism $\operatorname{Hom}(a, a) \simeq \mathbb{C}$ as an algebra. We assume for simplicity that the identity object 1 is simple.

We also assume that every object $x$ has a decomposition as a finite sum

$$
x=\bigoplus_{a} N_{a} a
$$

where $N_{a}$ is a nonnegative integer and $a$ is simple. In other words, every object $x$ is semisimple.

Finally, we assume finiteness, that is the number of isomorphism classes of simple objects is finite. Below, we will be somewhat cavalier on the distinction between simple objects and isomorphism classes of simple objects.

\subsubsection{Associativity structure}

The data in a symmetry category $\mathcal{C}$ includes certain isomorphisms implementing associativity of objects

$$
\alpha_{a, b, c} \in \operatorname{Hom}((a \otimes b) \otimes c, a \otimes(b \otimes c))
$$

which we call associators. ${ }^{4}$ Fusion matrices $F$ for the Moore-Seiberg data, and the (quantum) $6 \mathrm{j}$ symbols for the (quantum) groups are used in the literature to capture the data of associators.

The associator $\alpha_{a, b, c}$ corresponds to a local operator which implements the process of exchanging line $b$ from the vicinity of $a$ to the vicinity of $c$. See figure 2. They satisfy the pentagon identity which states the equality of following two morphisms

$$
\begin{aligned}
((a \otimes b) \otimes c) \otimes d & \rightarrow(a \otimes(b \otimes c)) \otimes d \rightarrow a \otimes((b \otimes c) \otimes d) \rightarrow a \otimes(b \otimes(c \otimes d)) \\
& =((a \otimes b) \otimes c) \otimes d \rightarrow(a \otimes b) \otimes(c \otimes d) \rightarrow a \otimes(b \otimes(c \otimes d))
\end{aligned}
$$

where each side of the equation stands for the composition of the corresponding associators. The pentagon identity ensures that exchanging two middle lines $b, c$ between two outer lines $a, d$ in two different ways is the same, see figure 3 .

\footnotetext{
${ }^{4}$ Since we can and do choose the identity morphisms $1 \otimes a \rightarrow a$ and $a \otimes 1 \rightarrow a$ to be trivial, the associator $\alpha_{a, b, c}$ is also trivial when any of $a, b, c$ is trivial.
} 


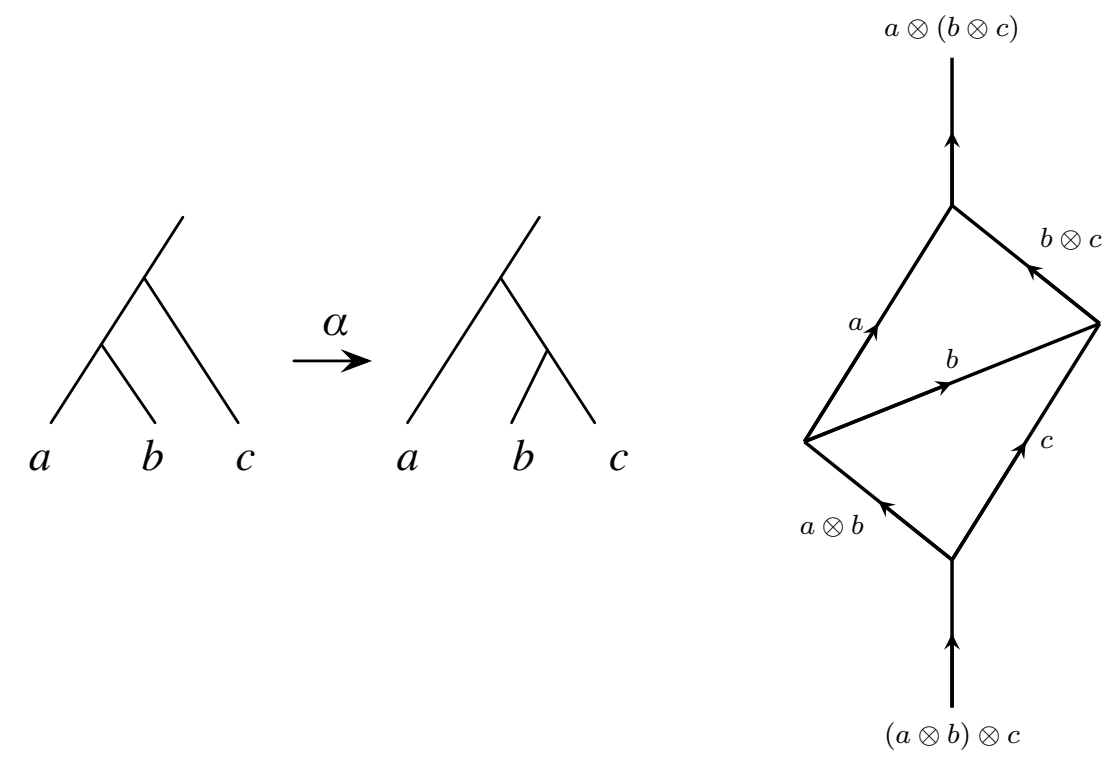

Figure 2. Left: the associator relates two different orders to tensor three lines. Right: equivalently, the local operator labeled by the associator morphism is obtained by squeezing the region between $(a \otimes b) \otimes c$ and $a \otimes(b \otimes c)$ shown above to a point.
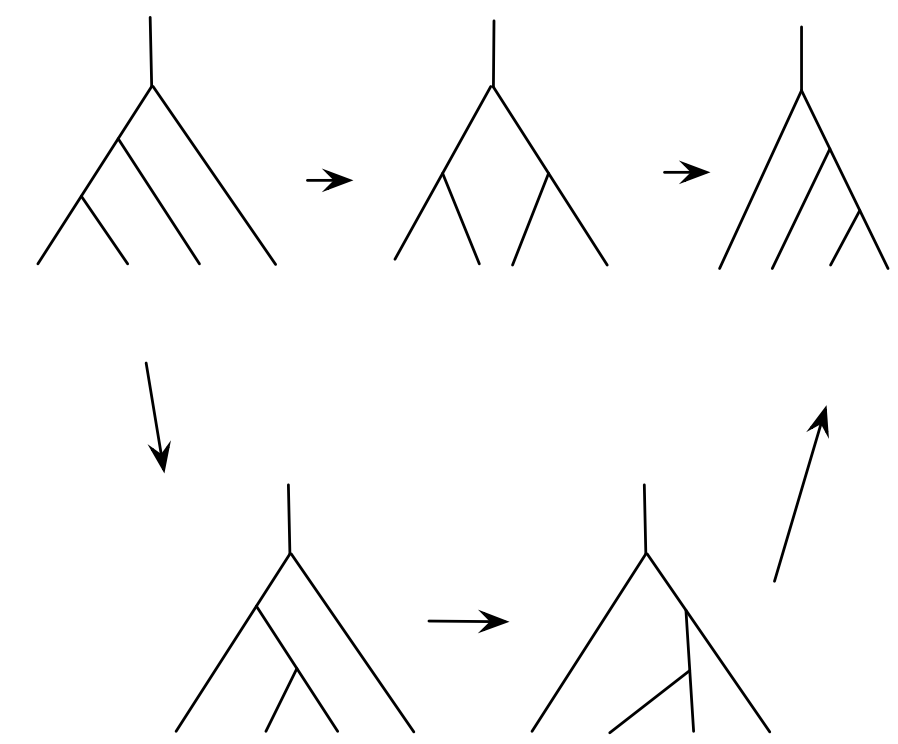

Figure 3. The pentagon identity guarantees that two distinct ways to rearrange the order of the tensoring of four lines lead to the same result. 


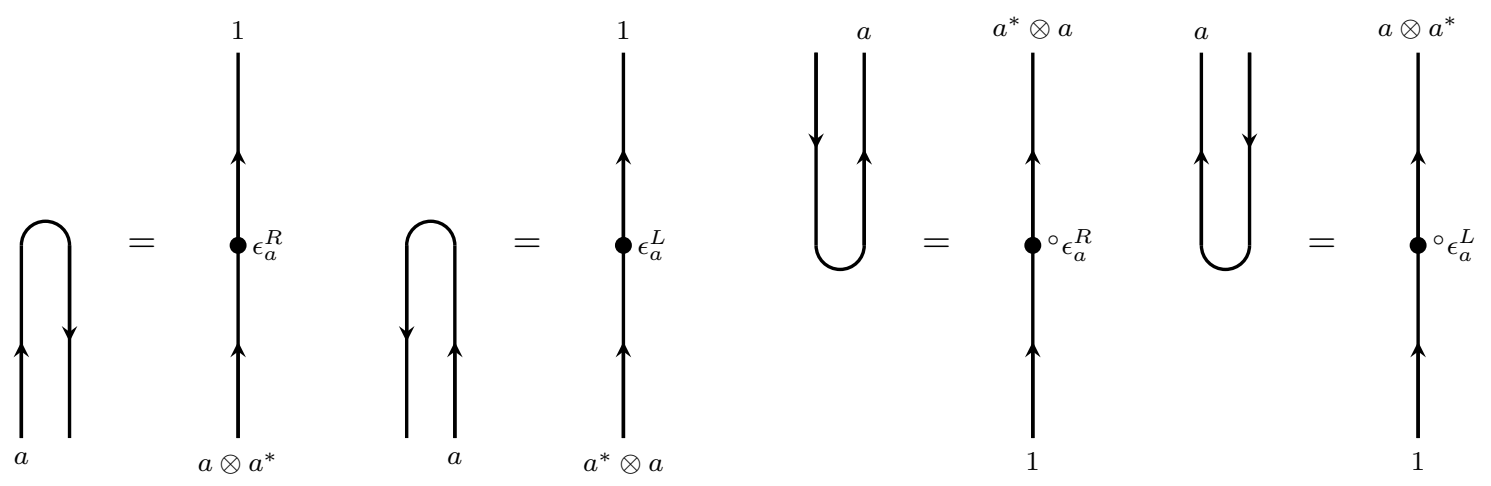

Figure 4. Folding a line and squeezing it gives rise to local operators labeled by evaluation and co-evaluation morphisms.

\subsubsection{Dual structure}

For every object $a, \mathcal{C}$ contains a dual object $a^{*}$. The line labeled by the dual object has the property that

$$
\langle\cdots a(C) \cdots\rangle=\left\langle\cdots a^{*}(\tilde{C}) \cdots\right\rangle .
$$

Here, $\tilde{C}$ denotes the same path $C$ but with a reverse orientation, and the morphisms attached at the junctions on $\tilde{C}$ need to be changed appropriately as we explain below at the end of this subsubsection.

We require that the dual of the dual is naturally isomorphic to the original object: $\left(a^{*}\right)^{*} \simeq a$. The dual operation also changes the order of the tensoring:

$$
(a \otimes b)^{*}=b^{*} \otimes a^{*} .
$$

We demand that there are evaluation morphisms

$$
\epsilon_{a}^{R}: a \otimes a^{*} \rightarrow 1, \quad \epsilon_{a}^{L}: a^{*} \otimes a \rightarrow 1
$$

and co-evaluation morphisms ${ }^{5}$

$$
{ }^{\circ} \epsilon_{a}^{R}: 1 \rightarrow a^{*} \otimes a, \quad{ }^{\circ} \epsilon_{a}^{L}: 1 \rightarrow a \otimes a^{*} .
$$

These label local operators corresponding to the process of folding a line operator $a$. See figure 4.

We note that $\epsilon_{a}^{R}$ and $\epsilon_{a^{*}}^{L}$ are not necessarily equal. However, we require as part of definition of dual structure that they are related as follows

$$
\epsilon_{a}^{R}=\epsilon_{a^{*}}^{L} \circ\left(p_{a} \otimes 1\right)=\epsilon_{a^{*}}^{L} \circ\left(1 \otimes p_{a^{*}}^{-1}\right)
$$

where $p_{a}$ is an isomorphism from $a$ to $a$ and $p_{a^{*}}$ is an isomorphism from $a^{*}$ to $a^{*}$. Similarly, we require

$$
{ }^{\circ} \epsilon_{a}^{R}=\left(1 \otimes p_{a}^{-1}\right) \circ{ }^{\circ} \epsilon_{a^{*}}^{L}=\left(p_{a^{*}} \otimes 1\right) \circ{ }^{\circ} \epsilon_{a^{*}}^{L}
$$

\footnotetext{
${ }^{5}$ We use the convention that when something is denoted by $x$, co-something is denoted by ${ }^{\circ} x$. This usage is unconventional, in particular for the case of coproduct for which $\Delta$ is definitely the standard notation, but it reduces the amount of notations that one has to remember.
} 


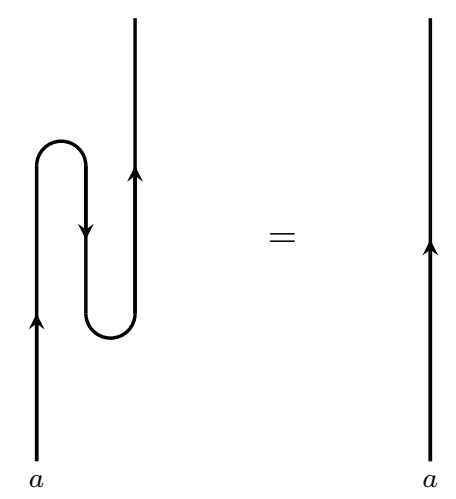

Figure 5. Consistency condition on evaluation and co-evaluation morphisms resulting from a topological deformation.

The data of $p_{a}$ and $p_{a^{*}}$ is referred to in the literature as a pivotal structure on the fusion category $\mathcal{C}$.

The evaluation and co-evaluation morphisms have to satisfy the following consistency condition with the associator

$$
\left(\epsilon_{a}^{R} \otimes 1\right) \circ \alpha_{a, a^{*}, a^{-1} \circ\left(1 \otimes{ }^{\circ} \epsilon_{a}^{R}\right)=1}
$$

as morphisms from $a$ to $a$. This ensures that a line with two opposite folds in the right direction can be unfolded as shown in figure 5. A similar identity is satisfied by $\epsilon_{a}^{L}$ and ${ }^{\circ} \epsilon_{a}^{L}$ which ensures that two opposite folds in the left direction can be unfolded.

Using evaluation and co-evaluation morphisms of different parity we can construct loops of lines

$$
\begin{gathered}
\left(\operatorname{dim}_{\mathrm{CC}} a\right) \text { id }: 1 \stackrel{{ }^{\circ} \epsilon_{a}^{R}}{\longrightarrow} a^{*} \otimes a \stackrel{\epsilon_{a}^{L}}{\longrightarrow} 1, \\
\left(\operatorname{dim}_{\mathrm{C}} a\right) \text { id }: 1 \stackrel{{ }^{\circ} \epsilon_{a}^{L}}{\longrightarrow} a \otimes a^{*} \stackrel{\epsilon_{a}^{R}}{\longrightarrow} 1 .
\end{gathered}
$$

These are morphisms from 1 to 1 and hence they are proportional to the identity morphism. The proportionality factors define two numbers: the counter-clockwise dimension $\operatorname{dim}_{\mathrm{CC}} a$ of $a$ and the clockwise dimension $\operatorname{dim}_{\mathrm{C}} a$ of $a$. See figure 6 .

Since we can replace the label $a$ by $a^{*}$ at the cost of flipping the orientation of line, we must have

$$
\begin{aligned}
\operatorname{dim}_{\mathrm{CC}} a & =\operatorname{dim}_{\mathrm{C}} a^{*}, \\
\operatorname{dim}_{\mathrm{CC}} a^{*} & =\operatorname{dim}_{\mathrm{C}} a .
\end{aligned}
$$

Indeed, this follows from (3.12) and (3.13).

In fact, it turns out that we can further argue that

$$
\operatorname{dim}_{\mathrm{CC}} a=\operatorname{dim}_{\mathrm{C}} a \equiv \operatorname{dim} a .
$$

To see this, place a small counter-clockwise loop of line $a$ around the "north pole" on the sphere. Let there be no other insertions anywhere on the sphere. This evaluates to 


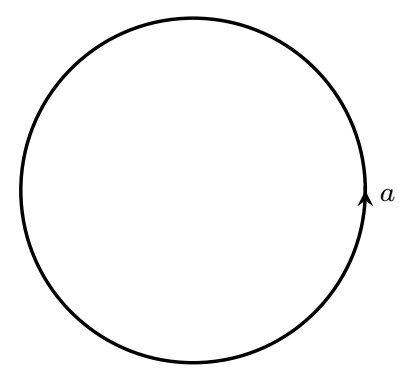

Figure 6. A loop of line $a$ constructed by composing evaluation and co-evalutaion morphisms. The loop, if it contains no other operators in it, can then be shrunk and the partition function with the loop is equal to $\operatorname{dim}_{\mathrm{CC}} a=\operatorname{dim} a$ times the partition function without the loop, with all other insertions unchanged.

$\operatorname{dim}_{\mathrm{CC}} a \times Z_{S^{2}}$ where $Z_{S^{2}}$ is the partition function on sphere. Now we can move the line such that it looks like a small clockwise loop around the "south pole" on the sphere. This evaluates to $\operatorname{dim}_{\mathrm{C}} a \times Z_{S^{2}}$. Equating the two expressions we find (3.19). ${ }^{6}$ This is a further constraint on $\mathcal{C}$. If the fusion category $\mathcal{C}$ satisfies (3.19), then $\mathcal{C}$ is called a spherical fusion category in the literature.

Since $\epsilon_{a}^{R}$ and $\epsilon_{a^{*}}^{L}$ are not necessarily equal, we have to specify whether a folding of line $a$ to the right should be read as the morphism $\epsilon_{a}^{R}$ or the morphism $\epsilon_{a^{*}}^{L}$. Similarly there is a specification of ${ }^{\circ} \epsilon_{a}^{R}$ vs. ${ }^{\circ} \epsilon_{a^{*}}^{L}$. This issue can be dealt with in two ways, which are technically equivalent but have a rather different flavor.

One method. One perspective is to regard that a line is always labeled by the pair (the local orientation, an object in $\mathcal{C})$. Then, a pair $(\uparrow, a)$ and $\left(\downarrow, a^{*}\right)$ are isomorphic but not actually the same. We note that this distinction needs to be made even when $a \simeq a^{*}$. Then we make the rule that when a vertical line is labeled by $(\uparrow, a)$ up to some point and then labeled by $\left(\downarrow, a^{*}\right)$ from that point, we insert the pivotal structure $p_{a} \in \operatorname{Hom}(a, a)$ at that point. This approach would be preferred by those who have no trouble with adding local orientations as a new datum to a topological line operator.

Another method. Another perspective is to think that the change between $\epsilon_{a}^{R}$ and $\epsilon_{a^{*}}^{L}$ and between ${ }^{\circ} \epsilon_{a}^{R}$ vs. ${ }^{\circ} \epsilon_{a^{*}}^{L}$ is canceled by changing the nearby morphisms. This method might be preferred by those who do not want to add local orientation as a new datum to a topological line operator.

We emphasize that, in this approach, the operation of exchanging $a$ by $a^{*}$ with a reversed orientation does not change the local operators at the junctions. Instead it changes the way the local operators at the junctions are read as morphisms in the associated symmetry category $\mathcal{C}$.

\footnotetext{
${ }^{6}$ The authors thank Shu-Heng Shao for discussion related to this point.
} 

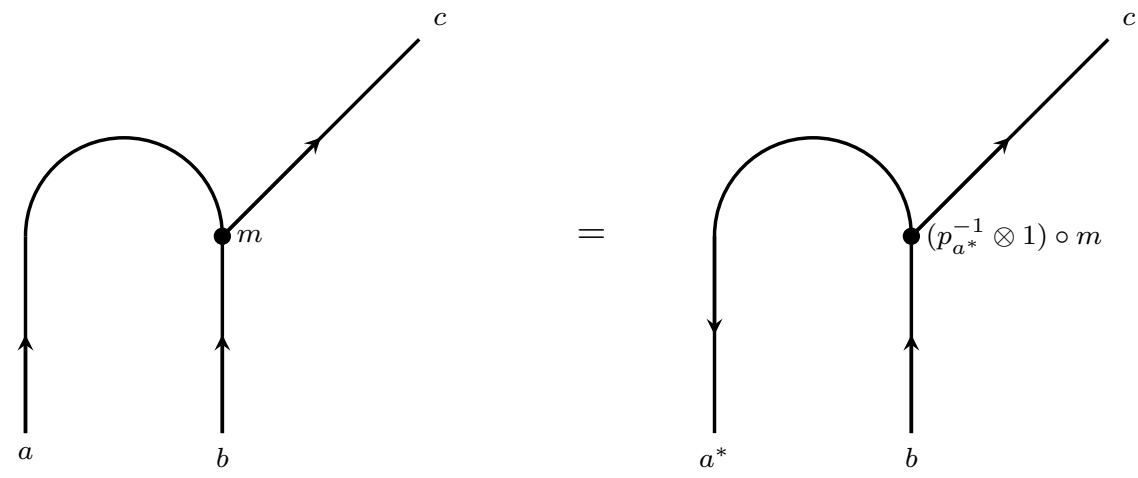

Figure 7. The fold in the diagram on left hand side is specified as $\epsilon_{a}^{R}$ and the fold on the right is specified as $\epsilon_{a^{*}}^{L}$. This changes the morphism from $m$ on the left side to $\left(p_{a^{*}}^{-1} \otimes 1\right) \circ m$ on the right side. These two diagrams provide two different categorical representations of the same physical configuration.

The following moves are sufficient to specify what happens in any situation:

1. Consider a morphism $\epsilon_{a}^{R} \circ \alpha_{a, a^{*}, c}^{-1} \circ(1 \otimes m): a \otimes b \rightarrow c$ where $m: b \rightarrow a^{*} \otimes c$. This is equal to $\epsilon_{a^{*}}^{L} \circ \alpha_{a, a^{*}, c}^{-1} \circ(1 \otimes n): a \otimes b \rightarrow c$ where $n=\left(p_{a^{*}}^{-1} \otimes 1\right) \circ m: b \rightarrow a^{*} \otimes c$.

2. Consider a morphism $\epsilon_{a^{*}}^{R} \circ \alpha_{c, a^{*}, a} \circ(m \otimes 1): b \otimes a \rightarrow c$ where $m: b \rightarrow c \otimes a^{*}$. This is equal to $\epsilon_{a}^{R} \circ \alpha_{c, a^{*}, a} \circ(n \otimes 1): b \otimes a \rightarrow c$ where $n=\left(p_{a^{*}} \otimes 1\right) \circ m: b \rightarrow c \otimes a^{*}$.

3. Consider a morphism $(1 \otimes m) \circ \alpha_{a^{*}, a, b} \circ{ }^{\circ} \epsilon_{a}^{R}: b \rightarrow a^{*} \otimes c$ where $m: a \otimes b \rightarrow c$. This is equal to $(1 \otimes n) \circ \alpha_{a^{*}, a, b} \circ{ }^{\circ} \epsilon_{a^{*}}^{L}: b \rightarrow a^{*} \otimes c$ where $n=m \circ\left(p_{a}^{-1} \otimes 1\right): a \otimes b \rightarrow c$.

4. Consider a morphism $(m \otimes 1) \circ \alpha_{b, a, a^{*}}^{-1} \circ{ }^{\circ} \epsilon_{a^{*}}^{R}: b \rightarrow c \otimes a^{*}$ where $m: b \otimes a \rightarrow c$. This is equal to $(n \otimes 1) \circ \alpha_{b, a, a^{*}}^{-1} \circ{ }^{\circ} \epsilon_{a}^{L}: b \rightarrow c \otimes a^{*}$ where $n=m \circ\left(1 \otimes p_{a}\right): b \otimes a \rightarrow c$.

These moves follow from (3.12) and (3.13). We draw a picture of the first move in figure 7. The other three moves are also described by similar pictures.

\subsubsection{Unitary structure}

The unitary structure requires an existence of a conjugate-linear involution sending $m \in \operatorname{Hom}(a, b)$ to $m^{\dagger} \in \operatorname{Hom}(b, a)$, generalizing the Hermitian conjugate in the standard linear algebra.

We require that the evaluation and the coevaluation morphisms are related by this conjugate operation:

$$
{ }^{\circ} \epsilon_{a^{*}}^{R}=\left(\epsilon_{a}^{L}\right)^{\dagger}, \quad{ }^{\circ} \epsilon_{a^{*}}^{L}=\left(\epsilon_{a}^{R}\right)^{\dagger} .
$$

We further require $m^{\dagger} \circ m \in \operatorname{Hom}(a, a)$ to be positive semi-definite in the following sense: since we assumed the semisimplicity and the finiteness of the number of simple objects, $\operatorname{Hom}(a, a)$ can naturally be identified with a direct sum of a matrix algebra. Then we require $m^{\dagger} \circ m$ to have non-negative eigenvalues.

The above positivity condition requires $\operatorname{dim} a>0$ for all $a$. As we will see in section 3.3.4 below, the unitarity implies sphericity. 


\subsection{Comments}

We have several comments:

- Note that category theorists do not like unitary structures, since it is specific to the base field $\mathbb{C}$ while they would like to keep everything usable for arbitrary base field. For this reason they often distinguish various concepts of $*$ operations and various structures satisfied by them, such as rigid structure, pivotal structure, spherical structure and pseudo-unitary structure. ${ }^{7}$ If we consider unitary $2 \mathrm{~d}$ quantum field theories (or more precisely its Wick-rotated versions which are reflection-positive), the unitary structure is the most natural one.

Operator algebraic quantum field theorists in fact work in this setting, since for them the existence of the positive-definite inner product on the Hilbert space is paramount. Unfortunately their papers often phrase purely categorical results in the operator algebra theoretic language, which makes them somewhat harder for outsiders to digest. From this point of view their review article [6] is very helpful, where a concise translation between terminologies of two different schools is given.

- Every property given above, except the simplicity of identity, semisimplicity and finiteness, is a straightforward expression of how topological lines and the junction operators associated to symmetries should behave. We impose the simplicity of identity, semisimplicity and finiteness to make the situation tractable. When the semisimplicity is dropped, the category is called a finite tensor category; when the simplicity of identity is dropped, the category is called a finite multi-fusion category; when both are dropped, it is called a finite multi-tensor category. When finiteness is dropped, we simply drop the adjective "finite".

Indeed, if we consider all topological lines in a given $2 \mathrm{~d}$ theory and all topological operators on topological lines, they might not in general form a unitary fusion category. Rather, our point of view is that we take a subset of topological lines and subspaces of topological operators on the lines so that they form a unitary fusion category, and then it can be thought of as a symmetry of the $2 \mathrm{~d}$ theory.

- A very similar categorical structure was introduced by Moore and Seiberg [11] in the analysis of $2 \mathrm{~d}$ RCFTs and 3d TFTs. In the category theory they are now called unitary modular tensor categories. In fact, the unitary modular tensor categories are also unitary fusion categories, where the latter description is obtained by forgetting the braiding.

\footnotetext{
${ }^{7}$ The rigid structure posits the existence of the left dual $a^{*}$ and the right dual ${ }^{*} a$, satisfying various conditions. It can be shown that ${ }^{* *} a \simeq a^{* *}$, and $a^{* * * *} \simeq a$. The pivotal structure is a collection of isomorphisms $a^{* *} \simeq a$. In our description, the pivotal structure relates $\epsilon_{a}^{L}$ and $\epsilon_{a^{*}}^{R}$. A pivotal structure is called spherical if $\operatorname{dim} a=\operatorname{dim} a^{*}$ for all $a$. A spherical structure is called pseudo-unitary if $|\operatorname{dim} a|$ is the largest eigenvalue of $\left(N_{a}\right)_{b}^{c}$ for all simple $a$. For the definition of $N_{a b}^{c}$, see section 3.3.3.
} 


\subsection{More notions of symmetry categories}

Before discussing examples, it is useful to set up a few more notions:

\subsection{1 'Homomorphisms' between symmetry categories}

In the case of two groups $G_{1}$ and $G_{2}$, we have the concept of homomorphisms $\varphi: G_{1} \rightarrow G_{2}$, preserving the group multiplication. Similarly, we can talk about symmetry functors $\varphi$ : $\mathcal{C}_{1} \rightarrow \mathcal{C}_{2}$ between two symmetry categories, together with the data specifying how the structures listed above are mapped. Among them are isomorphisms

$$
\epsilon_{a, b} \in \operatorname{Hom}(\varphi(a) \otimes \varphi(b), \varphi(a \otimes b))
$$

which tell us how the tensor structure of $\mathcal{C}_{1}$ is mapped into the tensor structure of $\mathcal{C}_{2}$. For example, the morphisms $\epsilon_{a, b}$ map the associator of $\mathcal{C}_{1}$ to the associator of $\mathcal{C}_{2}$.

Two symmetry functors $\varphi, \varphi^{\prime}: \mathcal{C}_{1} \rightarrow \mathcal{C}_{2}$ are considered equivalent when there is a set of isomorphisms

$$
\eta_{a} \in \operatorname{Hom}\left(\varphi(a), \varphi^{\prime}(a)\right)
$$

such that

$$
\eta_{a \otimes b} \epsilon_{a, b}=\epsilon_{a, b}^{\prime}\left(\eta_{a} \otimes \eta_{b}\right) .
$$

When a symmetry functor has an inverse, it is called an equivalence between symmetry categories.

\subsubsection{Products of symmetry categories}

In the case of two groups $G_{1}$ and $G_{2}$, their product $G_{1} \times G_{2}$ is also a group. Similarly, given two symmetry categories $\mathcal{C}_{1}$ and $\mathcal{C}_{2}$, we denote their product as $\mathcal{C}_{1} \otimes \mathcal{C}_{2}$, whose simple objects are given by $a_{1} \otimes a_{2}$ where $a_{1,2}$ are simple objects of $\mathcal{C}_{1,2}$, respectively. This product is called Deligne's tensor product of categories.

\subsubsection{Fusion rule of unitary fusion categories}

A symmetry category comes with a lot of structures. Sometimes it is useful to forget about most of them as follows. For each isomorphism class of simple objects $a$, introduce a symbol $[a]$, and define their multiplication by $[a][b]:=\sum_{c} N_{a b}^{c}[c]$ when $a \otimes b=\bigoplus_{c} N_{a b}^{c} c$. This makes non-negative integral linear combinations of $[a]$ 's into an algebra over $\mathbb{Z}_{+}$with a specific given basis. We call this algebra $R(\mathcal{C})$ the fusion ring of the symmetry category $\mathcal{C}$. In the case of modular tensor categories, this algebra is also called the Verlinde algebra. We would often call this algebra as just the fusion rule of $\mathcal{C}$.

Let $n$ be the number of isomorphism classes of simple objects. Then we can regard $\left(N_{a}\right)_{b}^{c}$ as $n \times n$ matrices and $[a] \mapsto N_{a}$ is the adjoint representation of the fusion ring.

\subsubsection{Determination of dimensions of objects}

The dimensions are fixed by $N_{a b}^{c}$. To see this, consider the $n$-dimensional vector $v:=(\operatorname{dim} a)_{a}$ where $a$ runs over the isomorphism classes of simple objects. Its entries are positive real numbers thanks to the unitarity. Furthermore, $v$ is the simultaneous eigenvector of all 
$N_{a}$ 's with eigenvalues $\operatorname{dim} a$. Then by the Perron-Frobenius theorem, $\operatorname{dim} a$ is the largest eigenvalue of the matrix $N_{a}$, which is guaranteed to be positive. The argument above applies both to $\operatorname{dim}_{\mathrm{C}}$ and $\operatorname{dim}_{\mathrm{CC}}$, and therefore the sphericity is implied by the unitarity. Unitarity also guarantees $\operatorname{dim} a=\operatorname{dim} a^{*}$.

We define the total dimension of the symmetry category $\mathcal{C}$ by the following formula:

$$
\operatorname{dim} \mathcal{C}=\sum_{a}(\operatorname{dim} a)^{2}
$$

Here the sum runs over the isomorphism classes of simple objects.

\subsection{Groups and representations of groups as symmetry categories}

\subsubsection{Symmetry categories $\mathcal{C}(G, \alpha)$}

As an example, let us recast an ordinary group $G$ as a symmetry category. We first regard each element $g \in G$ as a simple object denoted by the same letter in the category. We define

$$
g \otimes g^{\prime}:=g g^{\prime}, \quad g^{*}:=g^{-1} .
$$

Taking $\alpha_{g_{1}, g_{2}, g_{3}}$ to be the identity maps, they clearly form a unitary fusion category, which we denote by $\mathcal{C}(G)$.

More generally, the pentagon identity among $\alpha_{g_{1}, g_{2}, g_{3}}$ says that $\alpha$ is a 3 -cocycle on $G$ valued in $\mathrm{U}(1) .{ }^{8}$ Denote the resulting fusion category by $\mathcal{C}(G, \alpha)$. In the literature it is often denoted $\operatorname{Vec}_{G}^{\alpha}$. We clearly have $\operatorname{dim} g=1$. Thus the total dimension of this symmetry category is the order of the group.

When $\alpha_{1}$ and $\alpha_{2}$ differ by a coboundary of a 2-cochain $\epsilon$, we can construct an equivalence of categories between $\mathcal{C}\left(G, \alpha_{1}\right)$ and $\mathcal{C}\left(G, \alpha_{2}\right)$ using the functor specified using the same $\epsilon$ in (3.21). This means that in the definition of $\mathcal{C}(G, \alpha)$, one can regard $\alpha \in H^{3}(G, \mathrm{U}(1))$.

It is also clear that any unitary fusion category whose simple objects are all invertible can be made to be of this form. Summarizing,

A symmetry category $\mathcal{C}$ whose simple lines are all invertible is equivalent to $\mathcal{C}(G, \alpha)$ where $G$ is a finite group and $\alpha$ is an element in $H^{3}(G, \mathrm{U}(1))$.

\subsection{2 $\mathcal{C}(G, \alpha)$ and the anomaly}

As is by now familiar, this cohomology class $\alpha \in H^{3}(G, \mathrm{U}(1))$ specifies the anomaly of $G$ flavor symmetry in two dimensions $[12,13]$. One way to see it is as follows [2]: insert a network of lines with trivalent junctions between them on the spacetime manifold $\Sigma$. Let the lines be labeled by simple objects of $\mathcal{C}(G, \alpha)$, that is by group elements. And let every junction of the form $g \otimes g^{\prime} \rightarrow g g^{\prime}$ be labeled by the identity morphism $g g^{\prime} \rightarrow g g^{\prime}$. Such a configuration can also be thought of as reproducing the effects of a background connection on $\Sigma$ which has holonomies given by $g$ on crossing transversely a line labeled by $g$. Now, consider a local region looking like the left hand side of figure 8. Move the lines such

\footnotetext{
${ }^{8}$ As already noted in footnote 4 , in our convention $\alpha_{g_{1}, g_{2}, g_{3}}$ is trivial whenever any of $g_{1,2,3}$ is the identity. Such a cocycle is called normalized. It is a well-known fact in group theory that group cohomology can be computed by restricting every cochains involved to be normalized.
} 

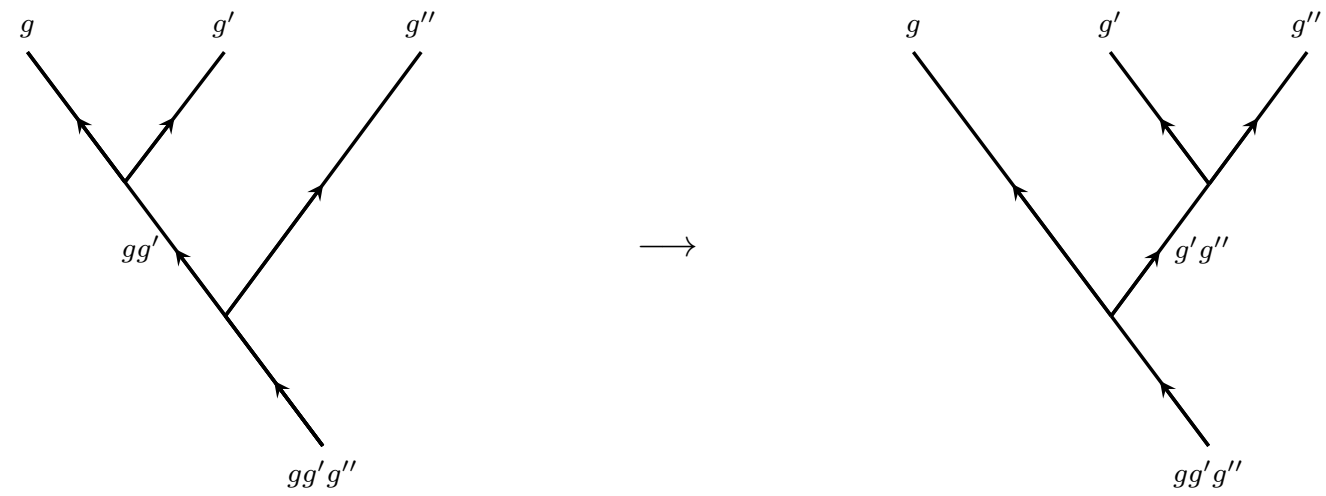

Figure 8. The same background connection represented in two different ways as networks of topological line operators. If the symmetry is anomalous, they lead to different partition functions.

that now it looks like the right hand side of figure 8. This changes the partition function by $\alpha\left(g, g^{\prime}, g^{\prime \prime}\right)$. The new background connection is just a gauge transform of the original background connection. Hence, we see that $\alpha$ precisely captures the anomaly in the flavor symmetry. Morally, this means the following:

\section{A symmetry category $\mathcal{C}$ includes the specification of its anomaly.}

Fixing a group $G$, the set of its anomalies forms an Abelian group. Notice that, in our language, $\mathcal{C}(G, \alpha)$ for different $\alpha$ have the same fusion ring $R=\mathbb{Z}_{+} G$. Thus, we can ask the following more general question: does the set of symmetry categories $\mathcal{C}$ with the same fusion ring $R$ form an Abelian group? The answer is that we need a coproduct on $R$. To see this, let us recall why the anomaly of a flavor symmetry forms an Abelian group from the perspective of quantum field theory.

In general, given two theories $T_{1,2}$, we can consider the product theory $T_{1} \times T_{2}$ which is just two decoupled theories considered as one. When $T_{i}$ has flavor symmetry group $G_{i}$, the product $T_{1} \times T_{2}$ has flavor symmetry group $G_{1} \times G_{2}$. When $G_{1}=G_{2}=G$, we can take the diagonal $G$ subgroup of $G \times G$ and regard $T_{1} \times T_{2}$ to have flavor symmetry $G$. Now, when $T_{i}$ has the anomaly $\alpha_{i}$, we define the anomaly of $T_{1} \times T_{2}$ to be the sum $\alpha_{1}+\alpha_{2}$. This abstractly defines the addition operation on the anomaly.

The crucial step that does not directly generalize to symmetry categories is the existence of the diagonal subgroup $G \subset G \times G$. In order to define the addition operation on the set of fusion categories sharing the same fusion rule $R$, similarly we need a coproduct $R \rightarrow R \otimes R$.

\subsection{3 $\mathcal{C}(G, \alpha)$ and the $G$-SPT phases}

Next, fixing a 3-cocycle $\alpha$, let us ask what is the autoequivalence of $\mathcal{C}(G, \alpha)$, that is, the self equivalence that preserves the structure as a symmetry category.

Pick an autoequivalence $\varphi: g \rightarrow \varphi(g)$ with the associated $\epsilon_{g, h} \in \operatorname{Hom}(\varphi(g) \varphi(h), \varphi(g h))$. Clearly $\varphi$ is an automorphism of $G$. Fixing $\varphi$ to be the identity, $\epsilon$ needs to be a 2-cocycle so that it does not change $\alpha$. Furthermore, two such $\epsilon$ 's are considered equivalent when 


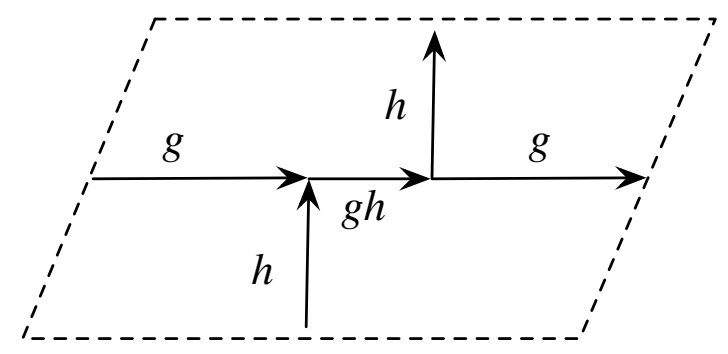

Figure 9. The lines representing a commuting holonomy $(g, h)$ on a torus.

they differ by a 2-coboundary, due to (3.23). Therefore $\epsilon$ can be thought of as taking values in $H^{2}(G, \mathrm{U}(1))$. Summarizing,

Autoequivalence of $\mathcal{C}(G, 0)$ is the semidirect product $\operatorname{Aut}(G) \ltimes H^{2}(G, \mathrm{U}(1))$.

The $\operatorname{Aut}(G)$ part is clear: it just amounts to renaming the topological lines associated to the group operation. How should we think of $H^{2}(G, \mathrm{U}(1))$ ? It is telling us that instead of choosing the identity operator as the implicit junction operator for $g \otimes g^{\prime} \rightarrow g g^{\prime}$ as done in figure 1 , we can choose $\epsilon_{g, g^{\prime}}$ times the identity. This will not change the associator but will change the partition function associated to a background connection on $\Sigma$. This corresponds to coupling a two-dimensional theory with $\mathcal{C}(G, \alpha)$ symmetry with a twodimensional bosonic symmetry protected topological (SPT) phase, which is specified by the 2-cocycle $\epsilon$ protected by the flavor symmetry $G$. The 2-cocycle $\epsilon$ is also known as a discrete torsion of $G$.

As an example, consider a torus with holonomies $g, h$ around two 1-cycles. They can be represented using the topological lines as in figure 9 . There, we resolved the intersection to two trivalent junctions.

We now change the operators at the two junctions to $\epsilon_{g, h}$ and $\epsilon_{g^{-1}, h^{-1}}$ given by the values of the 2 -cocycle. In total the phase of the partition function changes by

$$
c_{g, h}=\epsilon_{g, h} / \epsilon_{h, g}
$$

which is the standard relation between the discrete torsion phase $c$ on the torus and the 2-cocycle $\epsilon$ [14]. We can thus generalize as follows:

Autoequivalences of a symmetry category $\mathcal{C}$ generalize the notion of renaming and multiplying by SPT-phases for a group symmetry.

We need to keep in mind however that the phases introduced by $\epsilon$ in the general case do not have an interpretation of multiplying a SPT phase protected by $\mathcal{C}$, since the product of two theories with symmetry $\mathcal{C}$ has symmetry $\mathcal{C} \otimes \mathcal{C}$ but is not guaranteed to have symmetry $\mathcal{C}$, as already discussed above.

\subsection{4 $\operatorname{Rep}(G)$ as symmetry category}

Next, let us discuss $\operatorname{Rep}(G)$ for a finite group $G$. Its structure as a symmetry category is straightforward: the objects are representations of $G$, the morphism space $\operatorname{Hom}(R, S)$ 
between two representations consists of intertwiners, as the tensor product $\otimes$ and the associator $\alpha_{R, S, T}$ we use the ordinary operations, the dual is the complex conjugate, etc. The simple objects are the irreducible representations. The dimension of a representation $R$ as defined above equals the ordinary definition. Then, the total dimension of $\operatorname{Rep}(G)$ is the sum of the square of the dimensions of the irreducible representations, therefore

$$
\operatorname{dim} \operatorname{Rep}(G)=|G|
$$

Clearly, when $G$ is an Abelian group, we have a natural equivalence $\mathcal{C}(\hat{G})=\operatorname{Rep}(G)$. This means in particular that the symmetry categories $\operatorname{Rep}(G)$ for distinct Abelian $G$ are distinct. In fact the fusion rings $R(\operatorname{Rep}(G))$ are already distinct.

For non-Abelian $G$, the situation is more delicate. For example, take the dihedral group $D_{8}$ with eight elements (i.e. the symmetry of a square in three-dimensional space) and the quaternion group $Q_{8}$ (i.e. the group formed by eight quaternions $\pm 1, \pm i, \pm j, \pm k$ ). Their character tables are the same. In particular, they both have four one-dimensional irreducible representations $1, a, b$ and $a b$ and a unique two-dimensional irreducible representation $m$, with $m \otimes m \simeq 1 \oplus a \oplus b \oplus a b$. Therefore, their fusion rings are the same. In this case, however, the symmetry categories are different, since they are known to have distinct associator $\alpha_{m, m, m}$, see e.g. [15]. In this sense, the relation between $\operatorname{Rep}\left(D_{8}\right)$ and $\operatorname{Rep}\left(Q_{8}\right)$ is analogous to the relation between the symmetry categories $\mathcal{C}(G, \alpha)$ associated to the same group but with a different anomaly.

It is also known that there are cases where two distinct groups lead to the same symmetry category $[16,17]$. Let $\mathbb{F}_{2}$ be the finite field with two elements. The symplectic group $\operatorname{Sp}\left(2 d, \mathbb{F}_{2}\right)$ acts on $\left(\mathbb{F}_{2}\right)^{2 d}$. The extension of $\left(\mathbb{F}_{2}\right)^{2 d}$ by $\operatorname{Sp}\left(2 d, \mathbb{F}_{2}\right)$ with this natural action is classified by the cohomology $H^{2}\left(\operatorname{Sp}\left(2 d, \mathbb{F}_{2}\right),\left(\mathbb{F}_{2}\right)^{2 d}\right)$, see appendix A, in particular (A.11). It is known that when $d \geq 3$, there is a suitable nonzero $\nu \in H^{2}\left(\operatorname{Sp}\left(2 d, \mathbb{F}_{2}\right),\left(\mathbb{F}_{2}\right)^{2 d}\right)$ such that $G_{d}=\operatorname{Sp}\left(2 d, \mathbb{F}_{2}\right) \ltimes\left(\mathbb{F}_{2}\right)^{2 d}$. and $G_{d}^{\prime}=\operatorname{Sp}\left(2 d, \mathbb{F}_{2}\right) \ltimes_{\nu}\left(\mathbb{F}_{2}\right)^{2 d}$ we have $\operatorname{Rep}\left(G_{d}\right)=\operatorname{Rep}\left(G_{d}^{\prime}\right)$ as a symmetry category. The smallest example is when $d=3$, where $\left|G_{3}\right|=\left|G_{3}^{\prime}\right|=$ $\left(2^{6}-1\right)\left(2^{4}-1\right)\left(2^{2}-1\right) 2^{3^{2}} \cdot 2^{3 \cdot 2}=92897280$.

As already roughly discussed in section 2.2 , a theory $T / G$ where $T$ has flavor symmetry $G$ has a symmetry $\operatorname{Rep}(G)$, and re-gauging $\operatorname{Rep}(G)$ we get back the original theory $T$. Equivalently, if a theory $T^{\prime}$ has a symmetry $\operatorname{Rep}(G)$, gauging $\operatorname{Rep}(G)$ of $T^{\prime}$ has the symmetry $G$. In the example above, we said $\operatorname{Rep}\left(G_{d}\right)=\operatorname{Rep}\left(G_{d}^{\prime}\right)$ for different $G_{d}$ and $G_{d}^{\prime}$. This means that there are (at least) two distinct ways of gauging $\mathcal{C}=\operatorname{Rep}\left(G_{d}\right)=\operatorname{Rep}\left(G_{d}^{\prime}\right)$, producing two different theories with different symmetries $G_{d}$ and $G_{d}^{\prime}$. Let us next study what is going on in detail.

\section{Gaugings and symmetry categories}

Given a theory $T$ with non-anomalous $G$ symmetry, we can form the gauged theory $T^{\prime}=T / G$, and $T^{\prime}$ has $\operatorname{Rep}(G)$ as the symmetry category. We would like to precisely state the process of gauging $\operatorname{Rep}(G)$ of $T^{\prime}$ and getting back $T$. 
To do this, it is helpful first to state two procedures, one of obtaining $\operatorname{Rep}(G)$ from $G$ and another of obtaining $G$ from $\operatorname{Rep}(G)$, in a manner that treats $G$ and $\operatorname{Rep}(G)$ on an equal footing. This is done using the concept of module categories and bimodule categories.

Once we understand this process, it is straightforward to study how to gauge a more general symmetry category $\mathcal{C}$. We will also be able to answer how the symmetry category $\mathcal{C}$ changes under gauging in the general setting.

\subsection{Module and bimodule categories}

Readers would be familiar with the action of a group or an algebra on a vector space. Then the vector space is called a representation or equivalently a module. When there are two commuting actions, one from the left and another from the right, it is often called a bimodule. Module categories and bimodule categories are categorified versions of this construction. Our expositions will be brief; for more details, please consult chapter 7 of the textbook [7].

The categorified version of a linear space is an additive category. An additive category $\mathcal{M}$ is a category with the additive structure whose morphism spaces are vector spaces. Here and in the following, we assume objects of $\mathcal{M}$ to be of the form $\bigoplus_{m} N_{m} m$ where $m$ runs over simple objects and $N_{m}$ are non-negative integers. We also assume that the number of isomorphism classes of simple objects is finite.

The simplest additive category is Vec, the category of finite dimensional vector spaces. There is only one simple object $\mathbb{C}$, and every other object is isomorphic to $n \mathbb{C}=\mathbb{C}^{n}$.

Take $\mathcal{C}$ to be a symmetry category. A left-module category over $\mathcal{C}$ is an additive category $\mathcal{M}$ such that for $a \in \mathcal{C}$ and $m \in \mathcal{M}$ the product $a \otimes m \in \mathcal{M}$ is defined, together with the associativity morphisms

$$
\alpha_{a, b, m} \in \operatorname{Hom}((a \otimes b) \otimes m, a \otimes(b \otimes m))
$$

satisfying the pentagon identity. Direct sums of module categories can be easily defined. When $\mathcal{C}$ is a symmetry category it is known that every module category can be decomposed into a direct sum of indecomposable module categories.

Physically, a module category specifies a topological boundary condition. Each object $m$ in the module category specifies the boundary condition and the "type of flux" it carries. A line $a$ can end on $m$ at the cost of transforming $m$ to $a \otimes m$ which changes the flux at the boundary by $a$. If $a \otimes m \simeq m$ for all $a \in \mathcal{C}$ then $m$, along with its direct sums with itself, describes an indecomposable module category by itself which corresponds to a boundary condition that absorbs all the flux and hence we can refer to it as the "Dirichlet boundary condition". In general, an indecomposable module category is a generalization of mixed Dirichlet-Neumann boundary conditions familiar from gauge theory. In the literature, the structure on Vec of a module category for $\mathcal{C}$ is often called a fiber functor of $\mathcal{C}$, but we try not to use this terminology.

We can similarly define a right-module category over $\mathcal{C}$. A $\left(\mathcal{C}_{1}, \mathcal{C}_{2}\right)$ bimodule category is an additive category $\mathcal{M}$ which has a left action of $\mathcal{C}_{1}$ and a right action of $\mathcal{C}_{2}$, together with further compatibility morphisms

$$
\alpha_{c_{1}, m, c_{2}} \in \operatorname{Hom}\left(\left(c_{1} \otimes m\right) \otimes c_{2}, c_{1} \otimes\left(m \otimes c_{2}\right)\right) .
$$


A bimodule category has the physical interpretation that it describes a topological interface where lines in the symmetry categories $\mathcal{C}_{1,2}$ can end from the left and from the right, respectively.

Recall that a representation of an algebra $A$ on a vector space $V$ can be thought of as a homomorphism from $A$ to the algebra of matrices $\operatorname{Hom}(V, V)$. Similarly, given an additive category $\mathcal{M}$ we can form the symmetry category $\operatorname{Func}(\mathcal{M}, \mathcal{M})$ of additive self functors, and then giving the structure of a module category on $\mathcal{M}$ is the same as giving a homomorphism of symmetry categories from $\mathcal{C}$ to $\operatorname{Func}(\mathcal{M}, \mathcal{M})$.

The commutant of $\mathcal{C}$ within $\operatorname{Func}(\mathcal{M}, \mathcal{M})$ is denoted by $\mathcal{C}_{\mathcal{M}}^{*}$ and called the dual of $\mathcal{C}$ with respect to $\mathcal{M}$. When $\mathcal{M}$ is indecomposable, it is known that $\left(\mathcal{C}_{\mathcal{M}}^{*}\right)_{\mathcal{M}}^{*} \simeq \mathcal{C}$. $\mathcal{M}$ is naturally a $\left(\mathcal{C}, \mathcal{C}^{\prime}\right)$ bimodule category where $\mathcal{C}^{\prime}$ is $\mathcal{C}_{\mathcal{M}}^{*}$ with the order of the tensor product reversed. Since we will only use $\mathcal{C}^{\prime}$ and not $\mathcal{C}_{\mathcal{M}}^{*}$ in the following, we will call $\mathcal{C}^{\prime}$ the dual of $\mathcal{C}$ with respect to $\mathcal{M}$. Two symmetry categories $\mathcal{C}$ and $\mathcal{C}^{\prime}$ such that $\mathcal{C}^{\prime}$ is the dual of $\mathcal{C}$ acting on $\mathcal{M}$ are called categorically Morita equivalent. We will come back to this notion in section 4.7 .

\subsection{Duality of $\mathcal{C}(G)$ and $\operatorname{Rep}(G)$}

Let us discuss examples of module categories. We can give Vec a structure of a module category over $\mathcal{C}(G)$ : for $g \in \mathcal{C}(G)$ and $\mathbb{C} \in$ Vec, we just define $g \otimes \mathbb{C}=\mathbb{C}$, and let the associator be trivial. Note that this construction does not work for $\mathcal{C}(G, \alpha)$ with nonzero anomaly $\alpha \in H^{3}(G, \mathrm{U}(1))$, since the pentagon will not be satisfied.

We can also give Vec a structure of a module category over $\operatorname{Rep}(G):$ for $R \in \operatorname{Rep}(G)$ and $\mathbb{C} \in$ Vec, we just define $R \otimes \mathbb{C}=R \in$ Vec, and let the associator be the natural one induced from the tensor product structure of $G$. We note that for $\mathcal{C}=\operatorname{Rep}\left(G_{d}\right)=\operatorname{Rep}\left(G_{d}^{\prime}\right)$ at the end of section 3.4.4, the module category structures on $\operatorname{Vec}$ as $\operatorname{Rep}\left(G_{d}\right)$ and $\operatorname{Rep}\left(G_{d}^{\prime}\right)$ are different, since the associators (4.1) as morphisms in Vec turn out to be distinct.

Furthermore, we note that $\operatorname{Vec}$ is a $(\mathcal{C}(G), \operatorname{Rep}(G))$ bimodule category. The only additional ingredient is the associator

$$
\alpha_{g, \mathbb{C}, R} \in \operatorname{Hom}((g \otimes \mathbb{C}) \otimes R, g \otimes(\mathbb{C} \otimes R))=\operatorname{Hom}(R, R) .
$$

The compatibility condition just says that $\alpha_{g, \mathbb{C}, R}$ are representation matrices of $g$ on $R$, chosen compatible with the tensor product. Physically, we can regard Vec as the boundary between a system with $G$ symmetry on the left and a gauged system with $\operatorname{Rep}(G)$ symmetry on the right.

In fact, $\operatorname{Rep}(G)$ is characterized as the maximal set of right actions compatible with the left action of $\mathcal{C}(G)$, and $\mathcal{C}(G)$ as the maximal set of left actions compatible with the right action of $\operatorname{Rep}(G)$. As we will explicitly confirm in section 4.5, $\mathcal{C}(G)$ and $\operatorname{Rep}(G)$ are dual to each other with respect to Vec:

The symmetry category $\mathcal{C}(G)$ and $\operatorname{Rep}(G)$ are dual with respect to the module category Vec. 

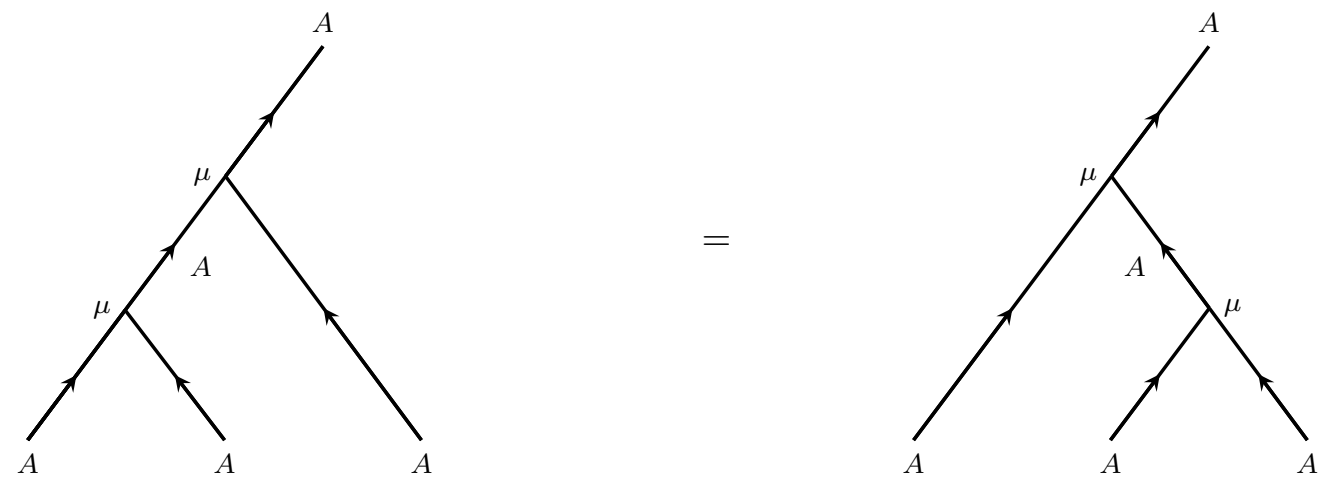

Figure 10. Associativity axiom for an algebra object $A \in \mathcal{C}$.
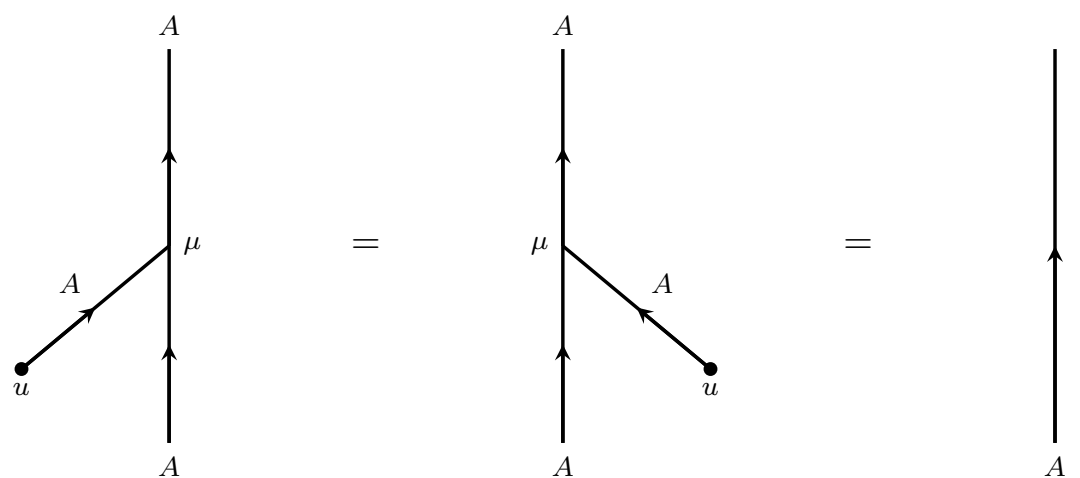

Figure 11. Unit axiom for an algebra object $A \in \mathcal{C}$.

\subsection{Gauging by an algebra object}

How is this duality of $\mathcal{C}(G)$ and $\operatorname{Rep}(G)$ related to gauging? To understand this, we need a digression. Take $A=\bigoplus_{g} g$ in $\mathcal{C}(G)$. The group multiplication gives a multiplication morphism $\mu: A \otimes A \rightarrow A$, and the map $1 \mapsto \bigoplus_{g} \delta_{1 g} g$ defines a unit morphism $u: 1 \rightarrow A$. These two operations satisfy the properties in figures 10 and 11. In general, if we have an object $A$ in a general symmetry category $\mathcal{C}$ along with morphisms $\mu$ and $u$ which satisfy these properties, then $A$ is called an algebra in $\mathcal{C}$.

We also have corresponding co-morphisms. The map $g \mapsto \frac{1}{|G|} \bigoplus_{h}\left(h \otimes h^{-1} g\right)$ defines a co-multiplication ${ }^{\circ} \mu: A \rightarrow A \otimes A$ and the projection $g \mapsto \delta_{1 g} 1$ defines a co-unit morphism ${ }^{\circ} u: A \rightarrow 1 .{ }^{\circ} \mu$ satisfies a co-associativity axiom which is given by turning the graphs in figure 10 upside down and changing the directions of all the arrows. Similarly, ${ }^{\circ} \mu$ and ${ }^{\circ} u$ together satisfy a co-unit axiom which is given by turning the graphs in figure 11 upside down and changing the directions of all the arrows. In general, if we have an object $A \in \mathcal{C}$ with morphisms ${ }^{\circ} \mu$ and ${ }^{\circ} u$ satisfying these axioms, we say that $A$ is a co-algebra in $\mathcal{C}$.

Moreover, in the group case we can explicitly check that $A$ satisfies additional properties which relate the algebra and co-algebra structure on $A$. These are shown in figures 12, 13 and 14. An object $A \in \mathcal{C}$ satisfying these properties is called as a symmetric Frobenius algebra object in $\mathcal{C}$. 


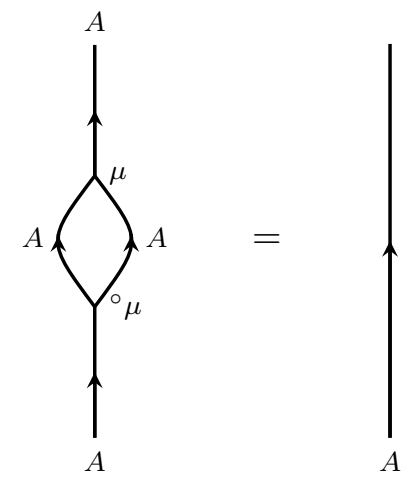

Figure 12. Separability axiom for an algebra object $A \in \mathcal{C}$.
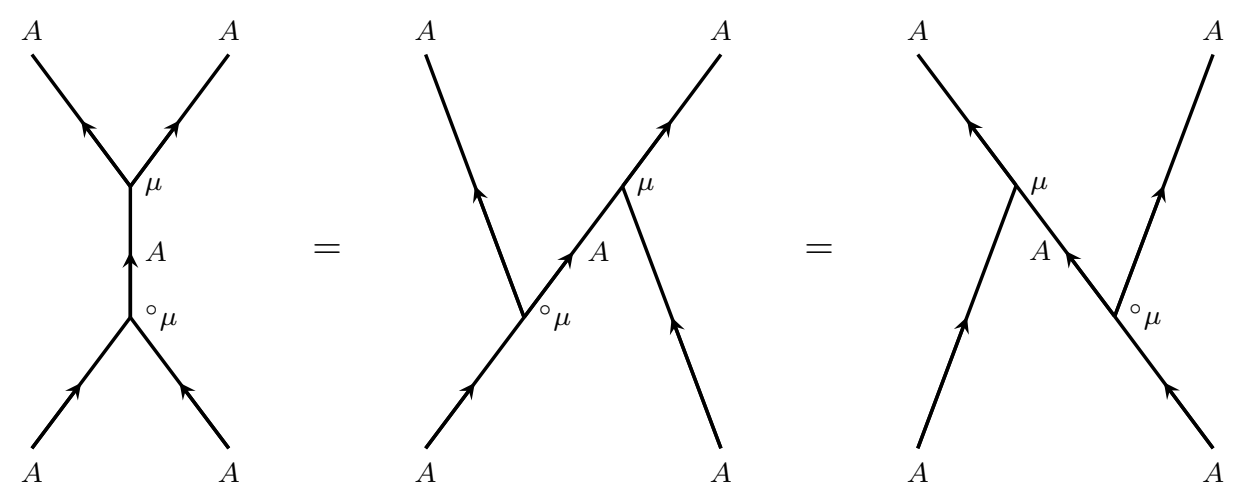

Figure 13. Frobenius axiom for an algebra object $A \in \mathcal{C}$.
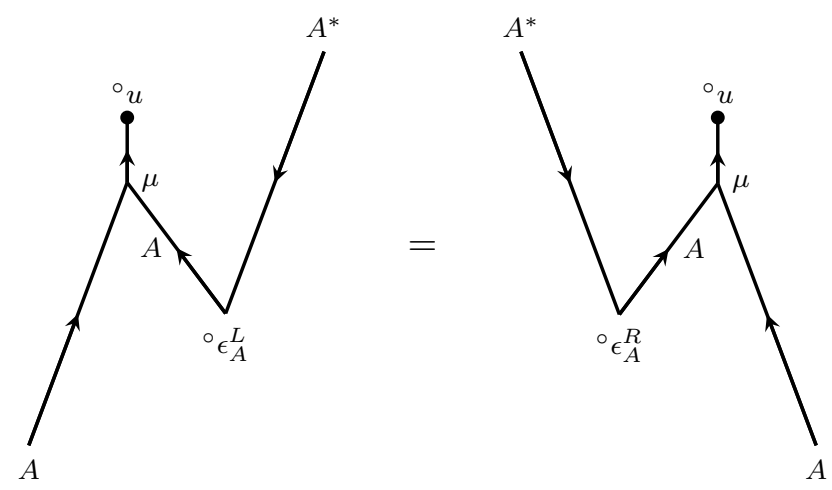

Figure 14. Symmetricity axiom for an algebra object $A \in \mathcal{C}$. Here ${ }^{\circ} \epsilon_{a}$ denotes the co-evaluation map for line $a$. 
Now, given a two-dimensional theory $T$ with flavor symmetry $G$, the partition function of the gauged theory $T / G$ can be described using this object $A$. Namely, $Z_{T / G}(M)$ on a manifold $M$ is defined by the partition function of $T$ on $M$ with a fine-enough trivalent mesh of topological lines all labeled by $A$. By fine-enough, we mean a mesh which can be obtained as the dual graph of a triangulation of the $2 \mathrm{~d}$ manifold $M$. The relations above guarantee that the result does not depend on the choice of the mesh as long as it is fine enough. Dually, these relations guarantee invariance under changes of triangulations by Pachner moves. Furthermore, by decomposing $A=\bigoplus_{g} g$, one can see that this trivalent mesh is a superposition of various $G$ bundles over $M$, giving us back the standard definition of the gauged theory.

The crucial idea of $[8,18]$ is to take this as the definition of gauging in the generalized sense. Namely, given a theory $T$ with a symmetry category $\mathcal{C}$, pick a symmetric Frobenius algebra object $A \in \mathcal{C}$. Then, the gauged theory $T / A$ is defined exactly as in the previous paragraph.

Note that there can be multiple possible choices of $A$ for a given $\mathcal{C}$. For example, when $\mathcal{C}=\mathcal{C}(G)$, we can pick any subgroup $H \subset G$ and take $A_{H}:=\bigoplus_{h \in H} h$. When $\mathcal{C}=\mathcal{C}(G, \alpha)$, we can check that $A_{H}$ is an algebra only when the anomaly $\alpha \in H^{3}(G, \mathrm{U}(1))$ when restricted to $H$ is trivial. We can also twist the multiplication morphism $m: A \otimes A \rightarrow A$ by using

$$
\epsilon_{g, h} \in \operatorname{Hom}(g \otimes h, g h)
$$

where $\epsilon_{g, h} \in H^{2}(G, \mathrm{U}(1))$. The choice of $A$ then can be thought of as the choice of a gaugeable subsymmetry together with the choice of the discrete torsion [3]. We summarize:

Gauging a gauge-able subpart of $\mathcal{C}$ is done by inserting a fine-enough mesh of an algebra object $A$ in $\mathcal{C}$. The choice of $A$ in $\mathcal{C}$ generalizes the notion of choosing a non-anomalous subgroup and the discrete torsion in the case of group symmetry.

We have some comments:

- There is in general no canonical maximal $A$ in $\mathcal{C}$. For example, given $\mathcal{C}(G, \alpha)$ for a nontrivial anomaly, there is not necessarily a unique maximal non-anomalous subgroup.

- In the case of $\mathcal{C}(G, \alpha)$, finding possible algebras $A$ is equivalent to finding a symmetry subcategory of the form $\mathcal{C}(H, 0)$. This is however not the general situation. Algebras $A$ specifying possible gaugings do not necessarily correspond to symmetry subcategories.

- Note also that two distinct $A$ and $A^{\prime}$ might give rise to the same gauged theory. We will come back to this question in section 4.6.

\subsection{Symmetries of the gauged theory from bimodules for the algebra object}

What is the symmetry category of the gauged theory $T / A$ ? Since $T / A$ is just the original theory $T$ with a fine mesh of $A$, we can still consider topological lines $p \in \mathcal{C}$ of the original 


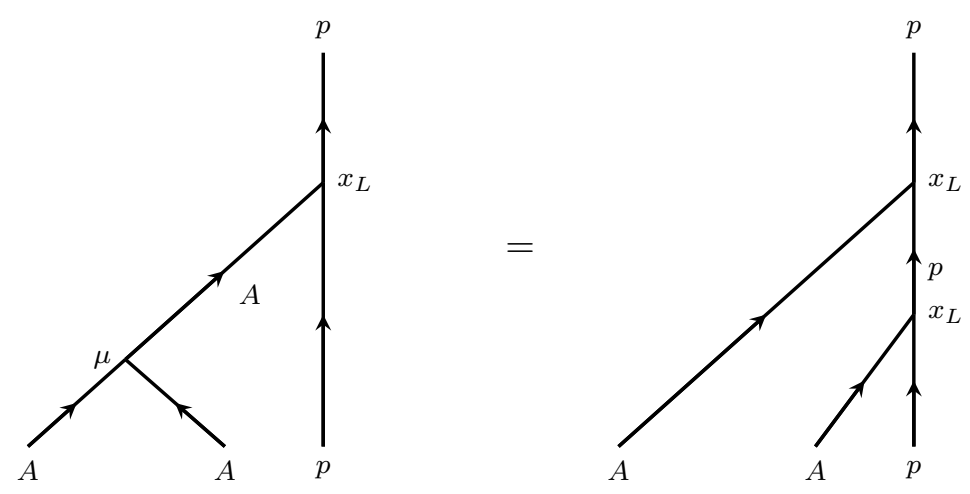

Figure 15. Associativity axiom for a left $A$-module $\left(p, x_{L}\right)$.

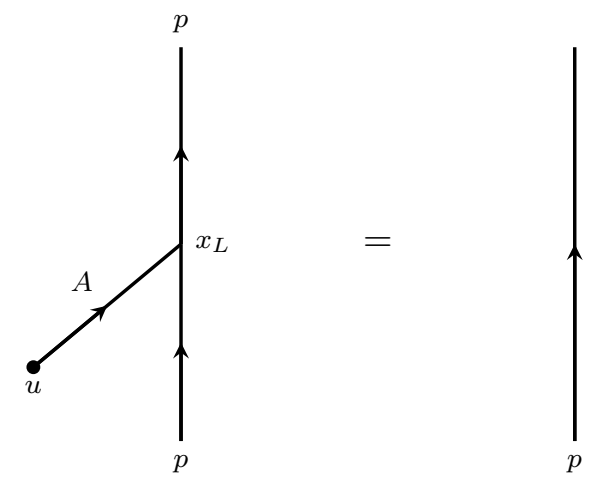

Figure 16. Unit axiom for a left $A$-module $\left(p, x_{L}\right)$.

theory. But for $p$ to be topological in the presence of an arbitrary mesh of $A$, the lines of $A$ need to be able to end on $p$ both on the left and the right consistently.

First of all, there must be a morphism $x_{L}: A \otimes p \rightarrow p$ such that conditions in figures 15 and 16 are satisfied. These properties make $p$ into a left $A$-module in $\mathcal{C}$. Similarly, there must be a morphism $x_{R}: p \otimes A \rightarrow p$ which satisfies similar conditions and makes $p$ into a right $A$-module as well. Moreover, there must be morphisms ${ }^{\circ} x_{L}: p \rightarrow A \otimes p$ and ${ }^{\circ} x_{R}: p \rightarrow p \otimes A$ which satisfy co-conditions obtained by reflecting the graphs in figures 15 and 16 upside down and reversing all the arrows. This would make $p$ into a left and right $A$-comodule. It turns out that for a symmetry category, we can always get the co-module structure by combining the module structure with the co-evaluation map for $A$ or $A^{*}$. Hence, from now on we restrict our attention only to the module structure. On top of all these conditions, $p$ must also satisfy conditions which allow us to commute the left and right actions of $A$ on $p$. This is the condition shown in figure 17 and it makes $p$ into an $(A, A)$-bimodule in $\mathcal{C}$.

The topological operators between two lines $p$ and $q$, both of which are bimodules, need to be compatible with the action of $A$ from both sides. That is, the insertion of the bimodule changing operator and the action of $A$ must commute. They give rise to the category $\operatorname{Bimod}_{\mathcal{C}}(A)$ of $(A, A)$ bimodules of $A$ within $\mathcal{C}$. Note that the concept of the 

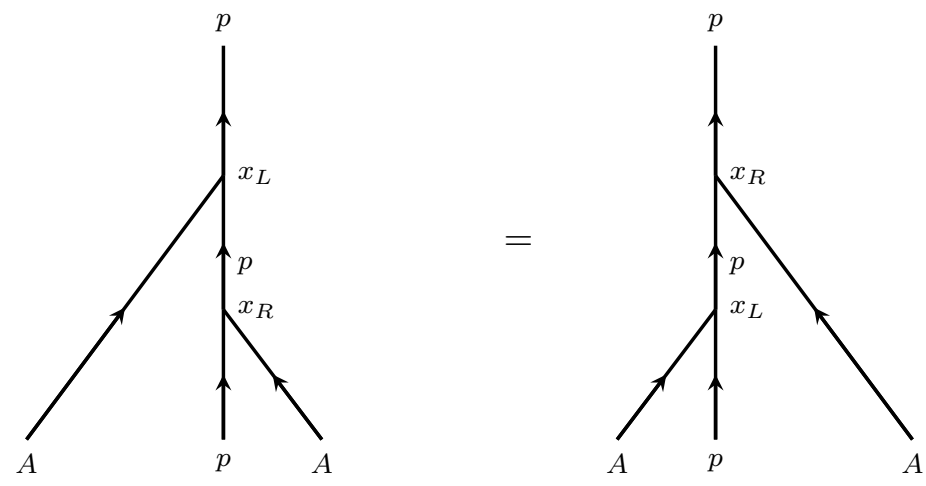

Figure 17. Definition of $(A, A)$-bimodule $\left(p, x_{L}, x_{R}\right)$.
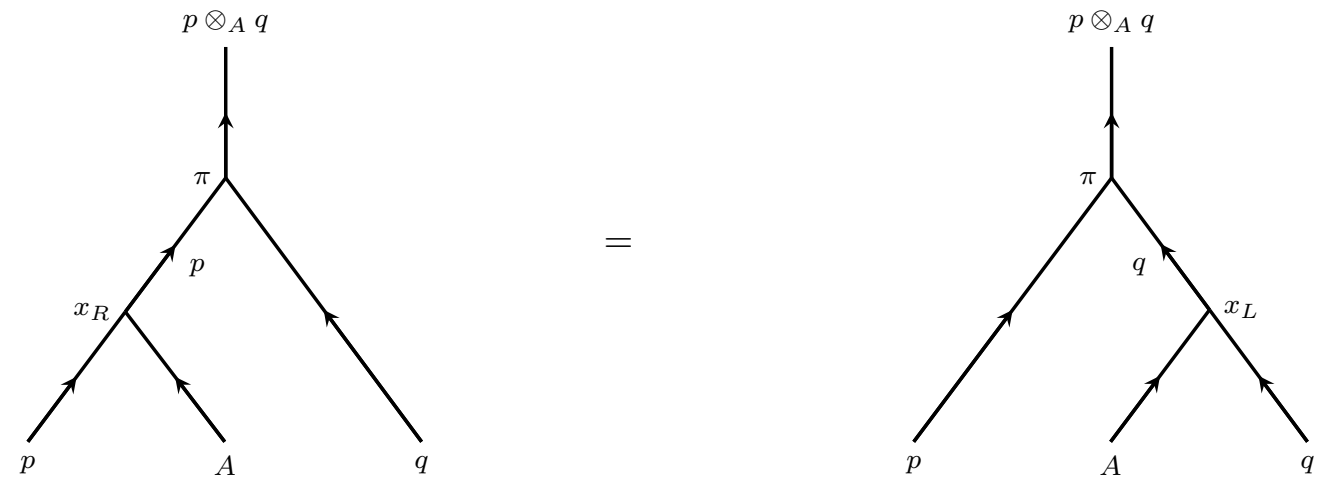

Figure 18. The compatibility condition defining balanced tensor product of two $A$-bimodules $p$ and $q$ via $\pi$.

category of $(A, A)$ bimodules is different from the concept of bimodule categories over $\mathcal{C}$ that we encountered above.

The tensor product in the category $\operatorname{Bimod}_{\mathcal{C}}(A)$ is written as $\otimes_{A}$ and it ensures that any insertions of $A$ between $p$ and $q$ in $p \otimes_{A} q$ can be removed. The product $p \otimes_{A} q$ is given as a subobject of $p \otimes q$ defined by the most general projection $\pi$

$$
\pi: p \otimes q \rightarrow p \otimes_{A} q
$$

which satisfies the equation

$$
(p \otimes A) \otimes q \stackrel{x_{R}}{\rightarrow} p \otimes q \stackrel{\pi}{\rightarrow} p \otimes_{A} q=(p \otimes A) \otimes q \stackrel{\alpha}{\rightarrow} p \otimes(A \otimes q) \stackrel{x_{L}}{\rightarrow} p \otimes q \stackrel{\pi}{\rightarrow} p \otimes_{A} q
$$

where each side of the equation stands for the composition of the morphisms shown, which are the associators $\alpha$, the projection $\pi$ and the morphisms $x_{L}$ and $x_{R}$ defining the action of $A$ on $p$ and $q$. See figure 18. The equation tells us that the right action of $A$ on $p$ is balanced against the left action of $A$ on $q$.

The left action of $A$ on $p \otimes_{A} q$ is defined by the compatibility condition

$$
A \otimes(p \otimes q) \stackrel{\pi}{\rightarrow} A \otimes\left(p \otimes_{A} q\right) \stackrel{x_{L}}{\rightarrow} p \otimes_{A} q=A \otimes(p \otimes q) \stackrel{\alpha}{\rightarrow}(A \otimes p) \otimes q \stackrel{x_{L}}{\rightarrow} p \otimes q \stackrel{\pi}{\rightarrow} p \otimes_{A} q
$$




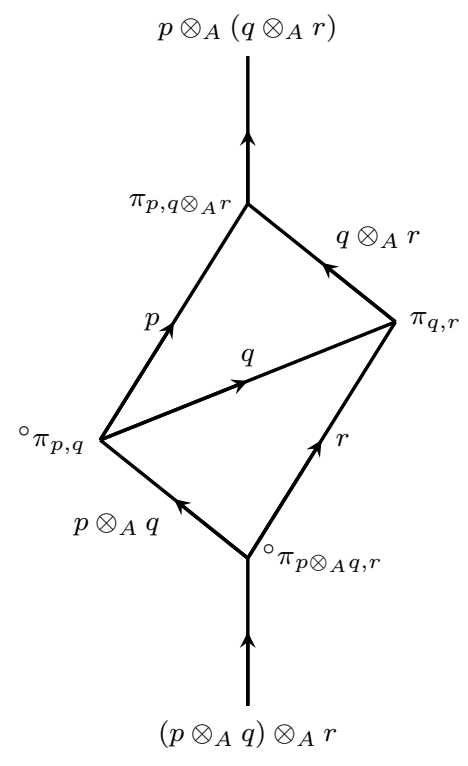

Figure 19. The associator in $\operatorname{Bimod}_{\mathcal{C}}(A)$.

where each side of the equation means the composition of the appropriate morphisms, namely the associators, the projection, and the left action of $A$ on $p$. The reader can draw a figure for (4.7) in a similar fashion as to figure 18 for (4.6). Similarly, we define the right action of $A$ on $p \otimes_{A} q$. These actions manifestly commute and convert $p \otimes_{A} q$ into an A-bimodule.

The dual of $\pi$ is ${ }^{\circ} \pi: p \otimes_{A} q \rightarrow p \otimes q$ and we have $\pi \circ{ }^{\circ} \pi=1$. The associator in $\operatorname{Bimod}_{\mathcal{C}}(A)$ is then defined as

$$
\tilde{\alpha}_{p, q, r}=\pi_{p, q \otimes_{A} r} \circ\left(1 \otimes \pi_{q, r}\right) \circ \alpha_{p, q, r} \circ\left({ }^{\circ} \pi_{p, q} \otimes 1\right) \circ\left({ }^{\circ} \pi_{p \otimes_{A} q, r}\right),
$$

see figure 19. This ensures that any insertions of $A$ can be removed in the diagram defining the associator.

Similarly, the evaluation and co-evaluation maps in $\operatorname{Bimod}_{\mathcal{C}}(A)$ are defined as $\tilde{\epsilon}_{p}^{L, R}=u \circ \epsilon_{p}^{L, R} \circ{ }^{\circ} \pi$ and ${ }^{\circ} \epsilon_{p}^{L, R}=\pi \circ{ }^{\circ} \epsilon_{p}^{L, R} \circ{ }^{\circ} u$ where $u$ and ${ }^{\circ} u$ are unit and co-unit morphisms defining $A$. The argument above suggests that we should take $\operatorname{Bimod}_{\mathcal{C}}(A)$ to be the symmetry category of the gauged theory $T / A$.

Now, consider two half-spaces, and put the mesh only on the right half. We now have a domain wall between the original theory $T$ on the left and the gauged theory $T / A$ on the right. On this domain wall, the lines of the theory $T$, in particular those from $\mathcal{C}$, should be able to end on the left and the lines of the theory $T / A$, namely those from $\operatorname{Bimod}_{\mathcal{C}}(A)$, should be able to end on the right. This suggests that the domain wall is described by the category $\operatorname{Mod}_{\mathcal{C}}(A)$, the category of right $A$-modules. Note that $\operatorname{Mod}_{\mathcal{C}}(A)$ has a natural left action of $\mathcal{C}$ and a natural right action of $\operatorname{Bimod}_{\mathcal{C}}(A)$, making it naturally a $\left(\mathcal{C}, \operatorname{Bimod}_{\mathcal{C}}(A)\right)$ bimodule category. Almost by definition, $\operatorname{Bimod}_{\mathcal{C}}(A)$ is the dual of $\mathcal{C}$ with respect to the module category $\operatorname{Mod}_{\mathcal{C}}(A)$. 


\subsection{Gauging of $\mathcal{C}(G)$ to get $\operatorname{Rep}(G)$ and vice versa}

As an exercise, let us describe the process explicitly when $\mathcal{C}=\mathcal{C}(G)$ and $\operatorname{Rep}(G)$.

From $\mathcal{C}(G)$ to $\operatorname{Rep}(G)$. We have already defined an algebra $A$ in $\mathcal{C}(G)$ in section 4.3. Let us determine $\operatorname{Mod}_{\mathcal{C}(G)}(A)$, the category of right modules of $A$.

Consider an object $M=\bigoplus N_{g} g$ where $N_{g}$ are non-negative integers. We constrain $M$ by demanding that it forms a right-module for $A$. First of all, we need a morphism $x: M \otimes A \rightarrow M$, which is characterized by morphisms $x_{g, g^{\prime}}: N_{g} g \otimes g^{\prime} \rightarrow N_{g g^{\prime}} g g^{\prime}$, each of which can be though of as an $N_{g g^{\prime}} \times N_{g}$ matrix. These matrices have to satisfy two equations. The first one involves the product axiom of $A$ and it tells us that

$$
x_{g g^{\prime}, g^{\prime \prime}} x_{g, g^{\prime}}=x_{g, g^{\prime} g^{\prime \prime}}
$$

The second one involves the unit axiom of $A$ and it tells us that

$$
x_{g, e}=1 .
$$

Combining these two, we find that

$$
x_{g, g^{\prime}} x_{g g^{\prime}, g^{\prime-1}}=1
$$

which implies that $N_{g}=N$ for all $g$ and

$$
x_{g, g^{\prime}}=x_{e, g g^{\prime}}\left(x_{e, g}\right)^{-1} .
$$

So, we find that the right modules are $M=N \bigoplus_{g} g=N A$ with arbitrary invertible $N \times N$ matrices $x_{e, g}$.

Now, we find the morphisms in $\operatorname{Mod}_{\mathcal{C}(G)}(A)$. The first observation is that all $\left(N A, x_{e, g}\right)$ are isomorphic to $(N A, 1)$. A morphism from right to left is provided by sending $N g$ inside $(N A, 1)$ to $N g$ inside $\left(N A, x_{e, g}\right)$ by the matrix $x_{e, g}$. The condition that this morphism commutes with the action of $A$ turns out to be (4.12) and hence this morphism is indeed a module morphism. The morphism from left to right via the inverse matrix is the inverse of this module morphism. So, we can restrict our attention to objects $N A \equiv(N A, 1)$.

Let us then find module morphisms from $N A$ to $N^{\prime} A$. Such a morphism $\varphi$ is specified by an $N \times N^{\prime}$ matrix $\varphi_{g}$ sending $N g$ inside $N A$ to $N^{\prime} g$ inside $N^{\prime} A$. The condition that $\varphi$ be a module morphism implies that $\varphi$ is a constant $N \times n^{\prime}$ matrix independent of $g$. Thus, we identify $\operatorname{Mod}_{\mathcal{C}(G)}(A)$ as the category Vec.

Next, let us determine $\operatorname{Bimod}_{\mathcal{C}(G)}(A)$. By a similar analysis as above, we can write such a bimodule as $\left(N A,\left(x_{L}\right)_{g, e},\left(x_{R}\right)_{e, g}\right)$ where $\left(x_{R}\right)_{e, g}$ and $\left(x_{L}\right)_{g, e}$ encode the morphisms $M \otimes A \rightarrow M$ and $A \otimes M \rightarrow M$. As before we can restrict our attention to $\left(N A,\left(x_{L}\right)_{g, e}, 1\right)$. Demanding that the left and right actions of $A$ are compatible, we find that

$$
\left(x_{L}\right)_{g, e}\left(x_{L}\right)_{g^{\prime}, e}=\left(x_{L}\right)_{g g^{\prime}, e}
$$

which means that the objects of $\operatorname{Bimod}_{\mathcal{C}(G)}(A)$ are identified as representations of $G$. One can check that the morphisms of $\operatorname{Bimod}_{\mathcal{C}(G)}(A)$ are precisely the intertwiners between the representations. Thus $\operatorname{Bimod}_{\mathcal{C}(G)}(A)$ is equivalent to $\operatorname{Rep}(G)$. We can also work out $\otimes_{A}$ and it agrees with the tensor product on $\operatorname{Rep}(G)$. 
From $\operatorname{Rep}(\boldsymbol{G})$ to $\mathcal{C}(\boldsymbol{G})$. Now the algebra object $A \in \operatorname{Rep}(G)$ is the regular representation. The regular representation is spanned by basis vectors $\widehat{g}$ in one-to-one correspondence with the group elements $g$. If we denote the action of $g$ by $\rho(g)$ then $\rho(g) \widehat{h}=\widehat{g h}$. The algebra multiplication takes $\widehat{g} \otimes \widehat{h}$ to $\delta_{g h} \widehat{g}$ which is an intertwiner. The unit morphism $1 \rightarrow A$ corresponds to choosing $\sum_{g} \widehat{g}$ in $A$.

First, we look for right modules of $A$. Choose some representation $R$ of $G$. We denote the action of $g$ on $\vec{q} \in R$ as $g \vec{q}$. Note that $g \vec{q} \neq 0$ for any $g$ and any non-zero $\vec{q}$ because otherwise this would imply that $\vec{q}=g^{|G|} \vec{q}=0$. The right-action of $A$ on $R$ must satisfy $g(\vec{p} \widehat{h})=(g \vec{p}) \widehat{h}$. We must also have that $(\vec{p} \widehat{h}) \widehat{k}=\delta_{h k} \vec{p} \widehat{h}$ and $\vec{p}\left(\sum_{g} \widehat{g}\right)=\vec{p}$. Now, start with some arbitrary vector $\vec{q} \neq 0$ such that $\vec{q} \widehat{g} \neq 0$ for some $g$. Then, it must be true that $\vec{p}:=\left(g^{-1} \vec{q}\right) \widehat{1} \neq 0$. Then, $\vec{p} \widehat{1}=\vec{p}$ and $\vec{p} \widehat{g}=0$ for all $g \neq 1$. This implies that for every $g$ we obtain a vector $\vec{p}_{g}=g \vec{p}$ which has the following properties: $\vec{p}_{g} \widehat{h}=\delta_{g h} \vec{p}_{g}$. Also, all $\vec{p}_{g}$ must be linearly independent because $\sum \alpha_{g} \vec{p}_{g}=0$ can be hit by $\widehat{h}$ from the right to yield $\alpha_{h}=0$. Thus the set formed by $\vec{p}_{g}$ for all $g$ is the regular representation $A$ of $G$ and $R$ breaks up as a sum of regular representations. Thus, the objects of $\operatorname{Mod}_{\operatorname{Rep}(G)}(A)$ are all isomorphic to $N A$ for some non-negative integer $N$. One can easily work out the module morphisms as well and then one finds that $\operatorname{Mod}_{\operatorname{Rep}(G)}(A)$ is equivalent to Vec.

Next, we look for bi-modules of $A$. Pick $A$ as a particular right module for $A$. It can be converted into a bimodule $V_{g}$ by simply stating that $\widehat{h} \vec{p}_{1}=\delta_{g h} \vec{p}_{1}$. The $G$-equivariance then determines that $\widehat{h} \vec{p}_{k}=\delta_{k g, h} \vec{p}_{k}$. Thus objects of $\operatorname{Bimod}_{\operatorname{Rep}(G)}(A)$ are labeled by nonnegative integers $n_{g}$ for each $g$. One can similarly figure out bimodule morphisms leading to the result that $\operatorname{Bimod}_{\operatorname{Rep}(G)}(A)$ is equivalent to $\mathcal{C}(G)$. Again, the tensor product $\otimes_{A}$ can be worked out and it equals the group multiplication.

\subsection{Gaugings and module categories}

Let us come back to the general question. We said that given a theory $T$ with a symmetry $\mathcal{C}$ and a symmetric Frobenius algebra object $A \in \mathcal{C}$, we can put a fine mesh of $A$ on the twodimensional manifold to define the gauged theory $T / A$. We now ask the question: when do two algebra objects $A$ and $A^{\prime}$ give rise to the same gauged theory $T / A \simeq T / A^{\prime}$ ? For this, the symmetries of the gauged theories should be the same, $\operatorname{Bimod}_{\mathcal{C}}(A) \simeq \operatorname{Bimod}_{\mathcal{C}}\left(A^{\prime}\right)$ and the properties of the domain walls should also be the same, $\operatorname{Mod}_{\mathcal{C}}(A) \simeq \operatorname{Mod}_{\mathcal{C}}\left(A^{\prime}\right)$.

Let us see that conversely, if $\operatorname{Mod}_{\mathcal{C}}(A) \simeq \operatorname{Mod}_{\mathcal{C}}\left(A^{\prime}\right)$, the gauged theories are the same $T / A \simeq T / A^{\prime}$. Indeed, the equivalence is captured by an $\left(A, A^{\prime}\right)$ bimodule $m$ in $\mathcal{C}$ which sends an object $M^{\prime}$ of $\operatorname{Mod}_{\mathcal{C}}\left(A^{\prime}\right)$ to an object $M$ of $\operatorname{Mod}_{\mathcal{C}}(A)$ via $M=m \otimes_{A^{\prime}} M^{\prime}$ where $\otimes_{A^{\prime}}$ denotes a tensor product in the category $\operatorname{Bimod}_{\mathcal{C}}\left(A^{\prime}\right)$. There is an inverse $\left(A^{\prime}, A\right)$ bimodule $n$ providing the inverse map. Physically, $m$ corresponds to a topological interface between $T / A$ and $T / A^{\prime}$ which can be fused with boundary conditions of $T / A^{\prime}$ to yield boundary conditions of $T / A . n$ is the inverse interface. Algebras $A$ and $A^{\prime}$ such that $\operatorname{Mod}_{\mathcal{C}}(A) \simeq \operatorname{Mod}_{\mathcal{C}}\left(A^{\prime}\right)$ are called Morita equivalent.

We claim that the existence of such an invertible interface guarantees that $T / A$ and $T / A^{\prime}$ are isomorphic theories. For instance, consider the Hilbert space $V$ of $T / A$ on $S^{1}$. It can be mapped to the Hilbert space $V^{\prime}$ of $T / A^{\prime}$ on $S^{1}$ by considering a cylinder geometry of infinitesimal time with the insertion of a wrapped domain wall $n$ in between. Similarly, 
we have an inverse map from $V^{\prime}$ to $V$ constructed similarly from $m$. Now, we can compose these maps by putting one cylinder on top of the other. As the interfaces are topological, they can be moved towards each other and ultimately fused away as they are inverses of each other. Thus, we are left with a unitary evolution on an infinitesimal length cylinder geometry. We can now take the infinitesimal to zero in all the steps above to obtain an isomorphism between $V$ and $V^{\prime}$. Similar arguments can be used to show that any kind of data of $T / A$ is isomorphic to the same kind of data of $T / A^{\prime}$.

We would like to stress that this equivalence of $T / A$ and $T / A^{\prime}$ is different from our claim that $T$ and $T / A$ contain the same amount of information. For example, in the latter case, the Hilbert spaces would be different, as we will detail in section 4.8.2.

All of this means that what really characterizes different gaugings are not the algebra objects used in the gaugings themselves but the associated module categories $\operatorname{Mod}_{\mathcal{C}}(A)$ over $\mathcal{C}$. We can then ask the question: does every module category $\mathcal{M}$ over $\mathcal{C}$ come from a symmetric Frobenius algebra object $A$ in $\mathcal{C}$, so that $\mathcal{M}$ arises as the domain wall between the original theory $T$ and the gauged theory $T / A$ ? The answer is yes [7, 19].

The construction goes roughy as follows. Given a module category $\mathcal{M}$ over $\mathcal{C}$, consider the morphism spaces $\operatorname{Hom}(c \otimes m, n)$ for $c \in \mathcal{C}$ and $m, n \in \mathcal{M}$. There is an object called the internal hom and denoted by $\underline{\operatorname{Hom}}(m, n)$ in $\mathcal{C}$ which satisfies

$$
\operatorname{Hom}_{\mathcal{M}}(c \otimes m, n) \simeq \operatorname{Hom}_{\mathcal{C}}(c, \underline{\operatorname{Hom}}(m, n)) .
$$

At the level of objects, this is given by

$$
\underline{\operatorname{Hom}}(m, n)=\bigoplus_{c}\left(\operatorname{dim} \operatorname{Hom}_{\mathcal{M}}(c \otimes m, n)\right) c
$$

where $c$ runs over the isomorphism classes of simple objects in $\mathcal{C}$ as can be simply verified by computing $\operatorname{Hom}_{\mathcal{C}}(c, \underline{\operatorname{Hom}}(m, n))$ from this equation.

Now, the internal homs can be concatenated naturally, in the sense that there is a natural morphism

$$
\mu: \underline{\operatorname{Hom}}(m, n) \otimes \underline{\operatorname{Hom}}(n, o) \rightarrow \underline{\operatorname{Hom}}(m, o)
$$

and in particular $\underline{\operatorname{Hom}}(m, m)$ has the multiplication. It can be shown [7] that $A=\underline{\operatorname{Hom}}(m, m)$ for a simple object $m \in \mathcal{M}$ is an algebra object such that $\operatorname{Mod}_{A}(C) \simeq \mathcal{M}$. Furthermore, $A$ is automatically symmetric Frobenius if $\mathcal{C}$ is a symmetry category [19]. Summarizing, we have

Distinct choices of gaugings of a theory with symmetry category $\mathcal{C}$ are in one to one correspondence with choices of indecomposable module categories $\mathcal{M}$ over $\mathcal{C}$ characterizing the domain wall between the original theory and the gauged theory. The gauged theory has the symmetry $\mathcal{C}^{\prime}$ which is the dual of $\mathcal{C}$ with respect to $\mathcal{M}$.

We denote the gauged theory corresponding to a module category $\mathcal{M}$ by $T / \mathcal{M}$. We would like to note that a decomposable module category corresponds to a direct sum of algebras $A=A_{1} \oplus A_{2}$ where the algebra structure of $A$ comes from algebra structure of $A_{1}$ and the algebra structure of $A_{2}$. Gauging a theory $T$ using $A$ produces $T / A=T / A_{1} \oplus T / A_{2}$ because a mesh of $A$ is the same as a mesh of $A_{1}$ plus a mesh of $A_{2}$. 


\section{7 (Re-)gauging and its effect on the symmetry category}

Now we can give a unified description of the (re-)gauging process. Consider a theory $T$ with a symmetry $\mathcal{C}$. Pick an indecomposable module category $\mathcal{M}$ of $\mathcal{C}$. There is an algebra object $A \in \mathcal{C}$ such that $\mathcal{M} \simeq \operatorname{Mod}_{\mathcal{C}}(A)$, and then the gauged theory $T / \mathcal{M}:=T / A$ has the symmetry $\mathcal{C}^{\prime}=\operatorname{Bimod}_{\mathcal{C}}(A)$ such that $\mathcal{M}$ is a natural $\left(\mathcal{C}, \mathcal{C}^{\prime}\right)$ bimodule category.

Now, pick an indecomposable module category $\mathcal{M}^{\prime}$ of $\mathcal{C}^{\prime}$ and repeat the same process. We get the gauged theory $T / \mathcal{M} / \mathcal{M}^{\prime}$ which has the symmetry $\mathcal{C}^{\prime \prime}$ such that $\mathcal{M}^{\prime}$ is a $\left(\mathcal{C}^{\prime}, \mathcal{C}^{\prime \prime}\right)$ bimodule category. We now see that the 'set' of all symmetry categories can be subdivided into 'subsets' consisting of symmetry categories that can be converted into each other by a sequence of gauging.

Note that this double gauging can be done in one step, since from two bimodule categories $\mathcal{M}$ and $\mathcal{M}^{\prime}$ we can form the tensor product $\mathcal{M} \nabla_{\mathcal{C}^{\prime}} \mathcal{M}^{\prime}$ which is a $\left(\mathcal{C}, \mathcal{C}^{\prime \prime}\right)$ bimodule category. Notationally it is convenient to write a $\left(\mathcal{C}, \mathcal{C}^{\prime}\right)$ bimodule category $\mathcal{M}$ as a morphism $\mathcal{M}: \mathcal{C} \rightarrow \mathcal{C}^{\prime}$. Then we can regard $\mathcal{M} \otimes_{\mathcal{C}^{\prime}} \mathcal{M}^{\prime}$ as $\mathcal{M}^{\prime} \circ \mathcal{M}$. Then $T / \mathcal{M} / \mathcal{M}^{\prime} \simeq T /\left(\mathcal{M}^{\prime} \circ \mathcal{M}\right)$. Any multiple gauging can be done in one step.

In particular, $\mathcal{M}^{\mathrm{op}}$ given by reversing the order of the tensor product is naturally a $\left(\mathcal{C}^{\prime}, \mathcal{C}\right)$ bimodule category, and $\mathcal{M}^{\mathrm{op}} \circ \mathcal{M}$ is the identity. Therefore we have $T / \mathcal{M} / \mathcal{M}^{\mathrm{op}}=T$. The example we started this paper with, $T / G / \hat{G}=T$ for an Abelian group $G$, is a special instance of this construction.

It is instructive to phrase the re-gauging process in terms of the algebra $A$ as well, whose detail can be found in [8] with plenty of helpful drawings. The category of bimodules $\operatorname{Bimod}_{\mathcal{C}}(A)$ have an algebra object $B=A^{*} \otimes A . B$ is also trivially an algebra in $\mathcal{C}$ and its algebra multiplication is given by the evaluation map. Moreover, we can embed $A$ into $B$ as $A \rightarrow A^{*} \otimes A \otimes A \rightarrow A^{*} \otimes A=B$ where the first map is the co-evaluation map and the second map is the multiplication for $A$. We are looking for $B$-bimodules in $\operatorname{Bimod}_{\mathcal{C}}(A)$ which can be identified with $B$-bimodules in $\mathcal{C}$ because $A$ embeds inside $B$ and hence a $B$ bimodule structure carries an $A$-bimodule structure. Now, $B$-bimodules in $\mathcal{C}$ are trivially obtained from any object $a \in \mathcal{C}$ by the mapping $A^{*} \otimes a \otimes A$. This mapping gives us an equivalence of $\mathcal{C}$ and the category of $B$-bimodules in $\operatorname{Bimod}_{\mathcal{C}}(A)$.

Physically, this means that the lines of $T / \mathcal{M} / \mathcal{M}^{\text {op }}$ come as lines of $T$ dressed by $A^{*}$ on one side and by $A$ on the other. The local operators in $T / \mathcal{M} / \mathcal{M}^{\text {op }}$ also correspond to a local operator of $T$ which appears at the junction of "middle" lines and the $A$-lines on the side are just joined smoothly. Let's insert a network of lines of $T / \mathcal{M} / \mathcal{M}^{\text {op }}$ on a surface $\Sigma$ and compute the partition function. The network will look like a network of lines of $T$ with additional $A$ loops, one for each "plaquette". These $A$ loops can be shrunk away leaving the partition function of a network of lines of $T$. Thus we see explicitly how we obtain the same theory on regauging. Later on, we will discuss 2 d TFTs with $\mathcal{C}$ symmetry and we will give a prescription for constructing the gauged TFT which uses heavily the algebra $A$ rather than $\mathcal{M}$. From this argument, it is clear that regauging the gauged TFT will give back the original TFT. 
Summarizing, we have the following:

Denote by $\mathcal{M}: \mathcal{C} \rightarrow \mathcal{C}^{\prime}$ when the symmetry category $\mathcal{C}$ has a gauging $\mathcal{M}$ under which the dual symmetry is $\mathcal{C}^{\prime}$. Two consecutive gaugings $\mathcal{M}: \mathcal{C} \rightarrow \mathcal{C}^{\prime}$ and $\mathcal{M}^{\prime}: \mathcal{C}^{\prime} \rightarrow \mathcal{C}^{\prime \prime}$ can be composed to $\mathcal{M}^{\prime} \circ \mathcal{M}: \mathcal{C} \rightarrow \mathcal{C}^{\prime \prime}$, and any gauging $\mathcal{M}: \mathcal{C} \rightarrow \mathcal{C}^{\prime}$ has an inverse $\mathcal{M}^{-1}: \mathcal{C}^{\prime} \rightarrow \mathcal{C}$ such that $\mathcal{M}^{-1} \circ \mathcal{M}$ is the identity.

Now, let us ask when are two symmetry categories $\mathcal{C}$ and $\mathcal{C}^{\prime}$ dual with respect to some indecomposable module category $\mathcal{M}$. It is known [20] that this happens if and only if $Z(\mathcal{C}) \simeq Z\left(\mathcal{C}^{\prime}\right)$, where $Z(\mathcal{C})$ is the Drinfeld center of $\mathcal{C}$. This has the following physical interpretation.

Recall first that any symmetry category where every simple object is invertible is of the form $\mathcal{C}=\mathcal{C}(G, \alpha)$. From the same data of $G$ and $\alpha \in H^{3}(G, \mathrm{U}(1))$, we can construct a 3d TFT called the Dijkgraaf-Witten theory [12]. On the boundary of the Dijkgraaf-Witten theory, we can put a 2 d theory with symmetry $G$ with an anomaly $\alpha$.

There is a generalization of this construction that gives a 3d TFT starting from any symmetry category $\mathcal{C}$, sometimes called the generalized Turaev-Viro construction. We will just call them the Dijkgraaf-Witten theory associated to $\mathcal{C}$. The Drinfeld center $Z(\mathcal{C})$ is the braided tensor category which captures the properties of the line operators of this $3 \mathrm{~d}$ TFT, and this $3 \mathrm{~d}$ TFT can have a boundary where a $2 \mathrm{~d}$ theory with symmetry $\mathcal{C}$ lives. For a recent exposition, see e.g. [21].

Since $T / A$ is obtained by just adding a mesh of $A$ on $T, T / A$ and $T$ can be put on boundaries of the same $3 \mathrm{~d}$ TFT. Therefore their Drinfeld centers should be the same. Conversely, specifying an isomorphism $Z(\mathcal{C}) \simeq Z\left(\mathcal{C}^{\prime}\right)$ corresponds to specifying a $1 \mathrm{~d}$ domain wall on a $2 \mathrm{~d}$ boundary of a single $3 \mathrm{~d}$ TFT, such that we have lines from $\mathcal{C}$ on the left and lines from $\mathcal{C}^{\prime}$ on the right of the domain wall. This corresponds to specifying a $\left(\mathcal{C}, \mathcal{C}^{\prime}\right)$ bimodule implementing the gauging. Summarizing,

Two symmetry categories $\mathcal{C}$ and $\mathcal{C}^{\prime}$ can be transformed to each other by an appropriate gauging if and only if the $3 d$ TFTs associated to them are equivalent, i.e. $Z(\mathcal{C}) \simeq Z\left(\mathcal{C}^{\prime}\right)$.

The fact $Z(\mathcal{C}) \simeq Z\left(\mathcal{C}^{\prime}\right)$ also implies that the total dimension of $\mathcal{C}$ and $\mathcal{C}^{\prime}$ are the same,

$$
\operatorname{dim} \mathcal{C}=\operatorname{dim} \mathcal{C}^{\prime}
$$

This is because it is known that $\operatorname{dim} Z(\mathcal{C})=|\operatorname{dim} \mathcal{C}|^{2}$.

Another related point is the following. This mathematical process of choosing a module category or an algebra object corresponding to it for a symmetry category and taking the dual category can be performed for a modular tensor category. A modular tensor category describes topological line operators in a three-dimensional theory and therefore this operation should be thought of as creating a new three-dimensional theory from an old one. This operation is in fact known under the name of anyon condensation [22].

\subsection{The effect of the gauging on Hilbert space on $S^{1}$}

Let us now discuss the Hilbert space on $S^{1}$ of a theory with symmetry $\mathcal{C}$, and how it is modified by the gauging. 


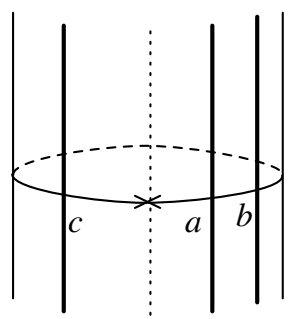

Figure 20. An example of a circle with transverse line operators. The point marked with an $\mathrm{X}$ labels the choice of the base point. In the diagrams here and below, the time flows upwards, and the lines also carry arrows in the upward direction unless otherwise mentioned.

\subsubsection{Backgrounds for $\mathcal{C}$ symmetry}

Consider a cylinder of the form $S^{1} \times \mathbb{R}_{t}$, with lines $a_{i}$ in $\mathcal{C}$ transverse to the constant time slice $t=0$. Let us assume that other data about the circle like its size, spin-structure etc. have been fixed so that we can associate a Hilbert space of states to this circle. See figure 20 for an example. In the figures here and below, the time flows from the bottom to the top.

We would like to identify this configuration of lines with an object $a$ of $\mathcal{C}$ and say that the circle carries the background a for the symmetry $\mathcal{C}$. When $\mathcal{C}=\mathcal{C}(G)$, the backgrounds are labeled by group elements and correspond to holonomy of the $G$-connection around the circle. As the lines are topological, we can try to define such an $a$ by fusing them together. Clearly, there are choices in the precise way one performs this procedure: there is a choice of the base-point from which we start taking the tensor product, and there is also a choice in the relative order of fusion of the lines, which is related to the associators in $\mathcal{C}$. To spell this out more fully, we need some notations and operations.

Let us denote the Hilbert space for a cylinder with just one line operator $a \in \mathcal{C}$ by $V_{a}$. We require $V_{a \oplus b}=V_{a} \oplus V_{b}$. A morphism $m: a \rightarrow b$ defines an operator $Z(m): V_{a} \rightarrow V_{b}$. This is given by the geometry shown in figure 21. Note that in a non-topological theory, the vertical height of the cylinder containing the topological line and/or the topological operator needs to be taken to zero. The same comment applies to all the figures discussed below in this section. Now, two choices of fusing $a, b$ and $c$ in figure 20 can be taken care of by using the associator $\alpha$ and $Z$ :

$$
Z\left(\alpha_{a, b, c}\right): V_{(a \otimes b) \otimes c} \rightarrow V_{a \otimes(b \otimes c)}
$$

To track the change of the base point, we need to introduce the map $X_{a, b}: V_{a \otimes b} \rightarrow V_{b \otimes a}$ defined by figure 22 , together with its inverse. They are associated to the intersection of the topological line and the auxiliary line, which is the trajectory of the base point.

The operations $X_{a, b}$ and $Z(\alpha)$ satisfy many consistency relations, which we spell out in full in section 6.2. With them, we can keep track of the change of the base point and the change of the order of fusing the lines in a consistent manner. This allows us to associate a Hilbert space to a circle with multiple transverse line operators $a_{1}, \ldots, a_{k}$. 


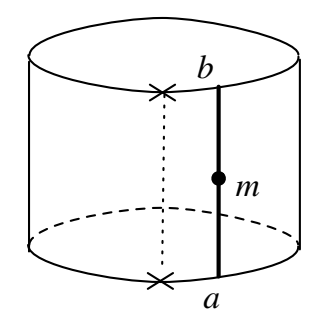

Figure 21. A morphism $m: a \rightarrow b$ defines an operator $Z(m): V_{a} \rightarrow V_{b}$.
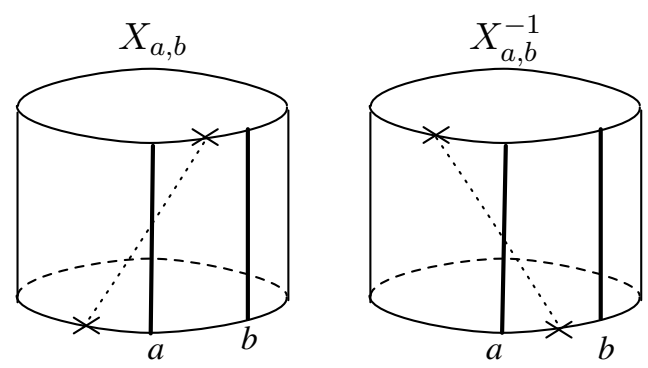

Figure 22. $X_{a, b}$ and its inverse tracks the change of the base points.

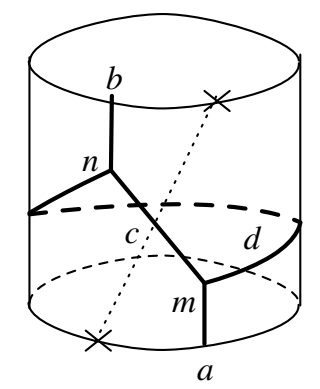

Figure 23. The action $U_{c, d}(m, n)$ of a line operator wrapped around $S^{1}$.

The operators $X$ and $Z$ can also be used to express how a line operator wrapped around $S^{1}$ acts on the Hilbert space. Indeed, given two morphisms $m: a \rightarrow c \otimes d$ and $n: d \otimes c \rightarrow b$, we can define the operator $U_{c, d}(m, n)$ using the network drawn in figure 23. Explicitly, this is given by

$$
U_{c, d}(m, n)=Z(n) X_{c, d} Z(m) .
$$

The action $U_{a}$ of a line operator labeled by $a$ wrapped around on $S^{1}$ on the Hilbert space $V_{1}$ on $S^{1}$ without any transverse line, referred to in section 3.1, is a special example of this construction: $U_{a}=U_{a^{*}, a}\left({ }^{\circ} \epsilon_{a}, \epsilon_{a}\right)$ where ${ }^{\circ} \epsilon_{a}, \epsilon_{a}$ are the co-evaluation map and the evaluation map introduced in (3.10), (3.11).

\subsubsection{The Hilbert space of the gauged theory}

So far we discussed the Hilbert spaces $V_{a}$ of the original theory $T$ with the symmetry $\mathcal{C}$, where $a \in \mathcal{C}$ is the label of the line operator transverse to the constant time slice $S^{1}$. Let us now discuss how Hilbert spaces $W_{p}$ of the gauged theory $T / A$ can be found, where $p \in \mathcal{C}^{\prime}$. 


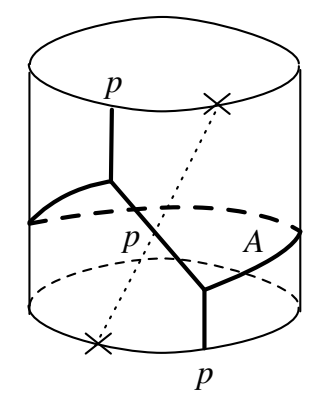

Figure 24. The projector defining the Hilbert space of the gauged theory is a specific instance of an action of a wrapped line operator. The morphisms used at the trivalent vertices are those specifying the bimodule structure of $p$.

A line in the gauged theory is given by an $(A, A)$ bimodule in $\mathcal{C}$. Each such bimodule $p$ can be viewed as an object in $\mathcal{C}$ and hence has a Hilbert space $V_{p}$ associated to it by the ungauged theory $T$. We construct the Hilbert space $W_{p}$ associated to $p$ by the gauged theory $T / A$ as a subspace of $V_{p}$ in the following manner.

In the case of $\mathcal{C}(G)$, one is traditionally instructed to project this space to the subspace left invariant by the action of the group $G$. For a general symmetry category $\mathcal{C}$ and its gauging $A$, we need a projector $P: V_{p} \rightarrow W_{p}$. Such a projector is naturally given by $P:=U_{p, A}\left({ }^{\circ} x_{R}, x_{L}\right)$ where ${ }^{\circ} x_{R}: p \rightarrow p \otimes A$ and $x_{L}: A \otimes p \rightarrow p$ are the morphisms defining the module and comodule structures on $p$, see figure 24 . This projector was written down in $[23]$.

Let us now show that this projector to $W_{p}$ agrees with the traditional definition in the case of $\mathcal{C}(G)$, when $p$ is the identity object in the category of bimodules. This means that $p=A=\bigoplus_{g} g$ as an object in $\mathcal{C}(G)$. Therefore we are going to project $V_{A}=\bigoplus_{g} V_{g}$. The operator $P$ restricted to $V_{0}$ is given by $|G|^{-1} \sum_{g} g$, and this is indeed a projector to the $G$-invariant subspace. The action of $P$ on the whole $V_{A}$ can be found similarly, and we find that

$$
W_{1}=P V_{A} \simeq \bigoplus_{C}\left(G_{c} \text {-invariant subspace of } V_{c}\right)
$$

where $C$ runs over conjugacy classes of $G, c \in C$ is a representative element, and $G_{c}$ is the commutant of $c$ in $G$. This is as it should be.

Summarizing, we have the following statement:

The Hilbert space of the gauged theory $T / A$ is obtained by taking the invariant part of the ungauged theory $T$ under the projector naturally defined by wrapping $A$ around $S^{1}$.

\section{More examples of symmetry categories and their gauging}

In the previous sections we reviewed the general theories of symmetry categories and their gauging. So far, however, we only saw basic examples where symmetry categories are either of the form $\mathcal{C}(G, \alpha)$ where $G$ is a finite group and $\alpha$ its anomaly, or of the form $\operatorname{Rep}(G)$ where $G$ is a group. In this section we discuss many other examples and their gauging. 


\subsection{Symmetry category with two simple lines}

The simplest symmetry category consists of just one simple object 1 and its multiples. This can be thought of as a symmetry of any $2 \mathrm{~d}$ theory, but it is not very interesting.

Let us then consider the simplest nontrivial symmetry category, consisting of simple objects 1 and $x$. The dual of $x$ can only be $x$, and then $x \otimes x$ can only contain one copy of 1 . Therefore the tensor product can only be of the form $x \otimes x=n x \oplus 1$, where $n$ is a non-negative integer.

When $n=0$, then the simple objects 1 and $x$ form the group $\mathbb{Z}_{2}$. As we already discussed, there are two possible symmetry categories $\mathcal{C}\left(\mathbb{Z}_{2}, \alpha\right)$ where $\alpha \in H^{3}\left(\mathbb{Z}_{2}, \mathrm{U}(1)\right)=\mathbb{Z}_{2}$ determines the anomaly.

When $n=1$, the dimension of $x$ is easily determined to be $(1+\sqrt{5}) / 2$. The only nontrivial condition is the pentagon identity of the associator $a_{x, x, x}$, and can be solved uniquely [24]. This symmetry category in fact has a braiding which turns it into a modular tensor category describing an anyon system. Recently this is known under the name of Fibonacci anyons because fusing $n$ copies of $x$ generates the $F_{n-1} 1 \oplus F_{n} x$ where $F_{n}$ is the $n$th Fibonacci number. For this reason we denote this category Fib.

It is known that we cannot have $n>1$, as shown by Ostrik [25]. Therefore, possible symmetry categories with two simple lines are just three, $\mathcal{C}\left(\mathbb{Z}_{2}, 0\right), \mathcal{C}\left(\mathbb{Z}_{2}, 1\right)$ and Fib. Ostrik also classified all possible symmetry categories with three simple objects [26].

\subsection{Symmetry category of SU(2) WZW models and other RCFTs}

Next, we review the symmetry category of RCFTs, following the construction of [27]. Let us take a rational chiral algebra $\mathcal{A}$ in two dimensions and consider a conformal field theory $T$ which corresponds to the diagonal modular invariant of this algebra $\mathcal{A}$. As is wellknown, from a chiral vertex operator $a$ corresponding to an irreducible representation of $\mathcal{A}$, we can construct a topological line operator $a$ of this theory $T$. Because the theory $T$ corresponds to the diagonal modular invariant, chiral and antichiral vertex operators generate the same line operators. Therefore the theory has topological lines generated by irreducible representations of a single copy of $\mathcal{A}$. They of course are specified by the Moore-Seiberg data, or equivalently they form a unitary modular tensor category $\mathcal{C}$. We can forget the braiding of $\mathcal{C}$ and regard it as the symmetry category of this theory $T$.

The essential observation of [27] is that the choice of gauge-able subpart of $\mathcal{C}$, or equivalently the choice of the module category $\mathcal{M}$ over $\mathcal{C}$, is in one to one correspondence with the choice of modular invariants of the chiral algebra $\mathcal{A}$. In particular, all modular invariants, including the exceptional ones, arise as the result of a generalized gauging.

Let us describe them in more detail in the case of SU(2) WZW models, following [28]. The chiral algebra is $\widehat{\mathrm{SU}}(2)_{k}$, which has $k+1$ irreducible representations

$$
V_{j}, \quad j=0,1 / 2, \cdots, k / 2
$$

with the fusion rule

$$
V_{j} \otimes V_{j^{\prime}}=V_{\left|j-j^{\prime}\right|} \oplus V_{\left|j-j^{\prime}\right|+1} \oplus \cdots \oplus V_{m}
$$


where $m=\min \left(j+j^{\prime}, k-\left(j+j^{\prime}\right)\right)$. We have

$$
\operatorname{dim} V_{j}=\frac{q^{j+1 / 2}-q^{-j-1 / 2}}{q^{1 / 2}-q^{-1 / 2}} \quad \text { where } \quad q=e^{2 \pi i /(k+2)} .
$$

They form a symmetry category we denote by $\operatorname{Rep}\left(\widehat{\mathrm{SU}}(2)_{k}\right)$. The object $V_{0}$ is the identity. It is clear that the objects $V_{j}$ with integral $j$ form a symmetry subcategory, and can be denoted by $\operatorname{Rep}\left(\widehat{\mathrm{SO}}(3)_{k}\right)$. In particular, when $k=3$, this is equivalent to the symmetry category Fib discussed above.

Since $V_{k / 2} \otimes V_{k / 2}=V_{0}$, the simple lines $V_{0}$ and $V_{k / 2}$ form a sub-symmetry category. From our general discussion above, this is equivalent to $\mathcal{C}\left(\mathbb{Z}_{2}, \alpha\right)$ where $\alpha \in H^{3}\left(\mathbb{Z}_{2}, \mathrm{U}(1)\right)=\mathbb{Z}_{2}$. This $\alpha$ is determined in terms of the associator, or equivalently the fusion matrix or the quantum $6 \mathrm{j}$ symbol involving four $V_{k / 2}$, and is known to be $\alpha=k \in \mathbb{Z}_{2}$. In particular $\operatorname{Rep}\left(\widehat{\mathrm{SU}}(2)_{1}\right)=\mathcal{C}\left(\mathbb{Z}_{2}, 1\right)$. This also means that the subsymmetry formed by $V_{0}$ and $V_{k / 2}$ is gauge-able when $k$ is even. Gauging it we obtain the modular invariant of type $D_{k / 2+2}$.

The $E_{6,7,8}$ modular invariants correspond to algebra objects

$$
A=\left\{\begin{array}{lll}
V_{0} \oplus V_{3}, & k=10 & \left(E_{6}\right), \\
V_{0} \oplus V_{4} \oplus V_{8}, & k=16 & \left(E_{7}\right), \\
V_{0} \oplus V_{5} \oplus V_{9} \oplus V_{14}, & k=28 & \left(E_{8}\right) .
\end{array}\right.
$$

The type $X_{n}$ of the modular invariants, or equivalently of the possible gauging, specifies the corresponding module category structure as follows: the isomorphism classes of simple objects in the module category are labeled by the nodes of the Dynkin diagram of $X_{n}$, and the edges describe how $V_{1 / 2}$ acts on the simple objects.

\subsection{Gauging a subgroup of a possibly-anomalous group}

\subsubsection{Generalities}

We now turn our attention to a more traditional setup of gauging a subgroup $H$ of a bigger group $G$. We will soon see that already in this traditional-looking setup we encounter various surprises.

We start by specifying the anomaly of the bigger group; we start from a symmetry category $\mathcal{C}(G, \alpha)$ where $\alpha \in H^{3}(G, \mathrm{U}(1))$. Possible gaugings are classified by their module categories, as already discussed. They turn out to be in one-to-one correspondence with a pair $(H, \psi)$ where $H$ is a subgroup such that the restriction of $\alpha$ to $H$ is trivial, and $\psi$ is an element of $H^{2}(H, \mathrm{U}(1)){ }^{9}$ This result agrees with the more traditional viewpoint: we choose a non-anomalous subgroup $H$ and then choose the discrete torsion $\psi$.

From the general machinery described above, the gauged theory has a symmetry category which is the dual of $\mathcal{C}(G, \alpha)$ with respect to $(H, \psi)$. Let us denote the resulting symmetry category by $\mathcal{C}^{\prime}:=\mathcal{C}(G, \alpha ; H, \psi)$. When $\alpha=0, H=G, \psi=0$, we already know that $\mathcal{C}^{\prime}=\operatorname{Rep}(G)$. Furthermore, when $G$ is abelian, $\mathcal{C}^{\prime}=\mathcal{C}(\hat{G}, 0)$. The explicit structure

\footnotetext{
${ }^{9}$ More precisely, when $\alpha$ is nontrivial, $\psi$ is an element of a torsor over $H^{2}(H, \mathrm{U}(1))$.
} 
of $\mathcal{C}^{\prime}$ in the general case can be determined by realizing it as a category of bimodules $\operatorname{Bimod}_{\mathcal{C}}(A)$ for the algebra object $A$ corresponding to $(H, \psi)$.

Let us see what we can say generally. Firstly, there are two general facts:

- the dimensions of lines in $\mathcal{C}^{\prime}$ are all integral; such symmetry categories are called as integral symmetry categories.

- the total dimension $\operatorname{dim} \mathcal{C}^{\prime}:=\sum_{a}(\operatorname{dim} a)^{2}$ is the same as the original one: $\operatorname{dim} \mathcal{C}^{\prime}=|G|$.

Secondly, there are cases where the dual symmetry $\mathcal{C}(G, \alpha ; H, \psi)$ itself is of the form $\mathcal{C}\left(G^{\prime}, \alpha^{\prime}\right)$ for some group $G^{\prime}$ and the anomaly $\alpha^{\prime}$. There is a theorem by Naidu, Nikshych $[29,30]$ and Uribe [31] determining exactly when this happens, and if so, explicitly the form of $G^{\prime}$ and $\alpha^{\prime}$. The general formula is too complicated to reproduce in full here. A necessary condition is that $H$ is an Abelian normal subgroup. When $\alpha$ is trivial this in fact suffices. In the next subsubsection we describe its explicit structure.

\subsubsection{Gauging a normal Abelian subgroup of a non-anomalous group}

Statement. Let us choose a group $G$ and its normal Abelian subgroup $H$. We then gauge $H$. The gauged theory then has a symmetry group $G^{\prime}$ with an anomaly $\alpha^{\prime} \in H^{3}\left(G^{\prime}, \mathrm{U}(1)\right)$, given as follows.

The fact that $H$ is a normal Abelian subgroup means that $G$ is an extension

$$
0 \rightarrow H \rightarrow G \rightarrow K=G / H \rightarrow 0
$$

and as such it is determined by an action of $K$ on $H$ by inner automorphisms in $G$, and an element $\kappa \in H^{2}(K, H)$ defined using the group action. The group $G$ is a crossed product, $G=H \rtimes_{\kappa} K$. Let us identity $G=H \times K$ as a set. Then the group structure is given as follows:

$$
(h, k)\left(h^{\prime}, k^{\prime}\right)=\left(h\left(k \triangleright h^{\prime}\right) \kappa\left(k, k^{\prime}\right), k k^{\prime}\right)
$$

where $k \triangleright h^{\prime}$ is the action of $k$ on $h^{\prime}$ and $\kappa\left(k, k^{\prime}\right)$ is an $H$-valued 2-cocycle of $K$.

Denote by $\hat{H}$ the dual group of $H$. There is a natural action of $K$ on $\hat{H}$ given by $k \triangleright \rho(h)=\rho\left(k^{-1} \triangleright h\right)$ for arbitrary elements $k \in K, h \in H$ and $\rho \in \hat{H}$. Under this action,

$$
G^{\prime}=\hat{H} \rtimes K
$$

with the trivial two-cocycle in $H^{2}(K, \hat{H})$, and $\alpha^{\prime}$ is given by

$$
\alpha^{\prime}=\alpha_{\kappa} \quad \text { where } \quad \alpha_{\kappa}\left((\rho, k),\left(\rho^{\prime}, k^{\prime}\right),\left(\rho^{\prime \prime}, k^{\prime \prime}\right)\right)=\rho^{\prime \prime}\left(k k^{\prime} \triangleright \kappa\left(k, k^{\prime}\right)\right)
$$

Note that the nontriviality $\kappa$ of the crossed product in the original $G$ side is traded for the nontriviality of the anomaly $\alpha_{\kappa}$ on the $G^{\prime}$ side. Summarizing, we have

Let us gauge a normal Abelian subgroup $H$ of a symmetry group $G$. $G$ is then necessarily of the form $G=H \rtimes_{\kappa} K$, where $\kappa \in H^{2}(K, H)$. When $G$ has no anomaly, the gauged theory has the symmetry group $G^{\prime}=\hat{H} \rtimes K$, and the resulting anomaly $\alpha^{\prime}$ is given in terms of $\kappa$ as in (5.8). 
Derivation. Let us now derive the description of $G^{\prime}$ given in the last paragraph. Our starting category is $\mathcal{C}(G)$ and we want to gauge it by the algebra object $A=\bigoplus h$ where $h \in H$. The simple objects of gauged category $\operatorname{Bimod}_{\mathcal{C}(G)}(A)$ are bimodules which can be seen to form the set $\hat{H} \times K$ using arguments very similar to those in section 4.5. An object $(\rho, k)$ in $\operatorname{Bimod}_{\mathcal{C}(G)}(A)$ is built from the object $\bigoplus_{h}(h, k)$ in $\mathcal{C}(G)$. Our choice of the bimodule structure on $(\rho, k)$ is that the right action by $A$ is trivial and the left action by $A$ is given in terms of morphisms $\left(x_{L}\right)_{h, h^{\prime}}:(h, e) \otimes\left(h^{\prime}, k\right) \rightarrow\left(h h^{\prime}, k\right)$ satisfying the familiar condition

$$
\left(x_{L}\right)_{h, h^{\prime}}=\left(x_{L}\right)_{h h^{\prime}, e}\left(\left(x_{L}\right)_{h^{\prime}, e}\right)^{-1}
$$

with

$$
\left(x_{L}\right)_{h, e}=\rho(h) .
$$

The balanced tensor product of $(\rho, k)$ and $\left(\rho^{\prime}, k^{\prime}\right)$ in $\operatorname{Bimod}_{\mathcal{C}(G)}(A)$ is given in terms of projectors $\pi_{h, h^{\prime}}:(h, k) \otimes\left(h^{\prime}, k^{\prime}\right) \rightarrow\left(h \kappa\left(k, k^{\prime}\right)\left(k \triangleright h^{\prime}\right), k k^{\prime}\right)$. The equation (4.6) tells us that

$$
\pi_{h\left(k \cdot h^{\prime}\right), e}=\pi_{h, h^{\prime}} \rho^{\prime}\left(h^{\prime}\right) .
$$

Demanding the right action on $(\rho, k) \otimes_{A}\left(\rho^{\prime}, k^{\prime}\right)$ to be trivial leads us to the condition that

$$
\pi_{h, h^{\prime}}=\pi_{h, e}
$$

which can be substituted into (5.11) to simplify it to

$$
\pi_{h\left(k \triangleright h^{\prime}\right), e}=\pi_{h, e} \rho^{\prime}\left(h^{\prime}\right) .
$$

Via (4.7), the left action $\rho^{\prime \prime}$ on $(\rho, k) \otimes_{A}\left(\rho^{\prime}, k^{\prime}\right)$ satisfies

$$
\rho^{\prime \prime}(h) \pi_{e, e}=\pi_{h, e} \rho(h)
$$

which can be combined with (5.13) to yield

$$
\rho^{\prime \prime}(h)=\rho(h) \rho^{\prime}\left(k^{-1} \triangleright h\right) .
$$

In particular we have

$$
\pi_{h, e}=\rho^{\prime}\left(k^{-1} \triangleright h\right) .
$$

The equation (5.15) means that $\operatorname{Bimod}_{\mathcal{C}(G)}(A)$ is equivalent to $\mathcal{C}\left(G^{\prime}, \alpha^{\prime}\right)$ where $G^{\prime}=\hat{H} \rtimes K$ for some yet to be determined $\alpha^{\prime}$.

The associator can be computed from the graph in figure 19. Let the objects $p, q, r$ be $(k, \rho),\left(k^{\prime}, \rho^{\prime}\right),\left(k^{\prime \prime}, \rho^{\prime \prime}\right)$. It suffices to restrict each object to the sub-object $(k, e)$ in $\mathcal{C}(G)$. Without loss of generality, we can assume $\pi_{e, e}=1$ because factors of $\pi_{e, e}$ are canceled by factors of ${ }^{\circ} \pi_{e, e}$. Then the only contribution comes from what is denoted as ${ }^{\circ} \pi_{p \otimes_{A} q, r}$ in figure 19 and we find the anomaly $\alpha^{\prime}$ given in (5.8). 
Examples. As an example, consider $G=\mathbb{Z}_{2 n}$ generated by $x$ with $x^{2 n}=1$, and gauge the $\mathbb{Z}_{2}$ subgroup generated by $x^{n}$. When $n$ is odd, $G=\mathbb{Z}_{2} \times \mathbb{Z}_{n}$, and the dual symmetry is clearly just $G^{\prime}=\hat{\mathbb{Z}}_{2} \times \mathbb{Z}_{n}$ without any anomaly, since the part $\mathbb{Z}_{n}$ does not matter. When $n$ is even, $G$ is a nontrivial extension $0 \rightarrow \mathbb{Z}_{2} \rightarrow G=\mathbb{Z}_{2 n} \rightarrow \mathbb{Z}_{n} \rightarrow 0$, corresponding to a nonzero $\kappa \in H^{2}\left(\mathbb{Z}_{n}, \mathbb{Z}_{2}\right)$. This means that the dual is $G^{\prime}=\hat{\mathbb{Z}}_{2} \times \mathbb{Z}_{n}$, with a nontrivial anomaly $\alpha_{\kappa}$ as given above.

As another example, consider $G=D_{2 n}$, the dihedral group of $2 n$ elements, generated by two elements $r, s$ such that $r^{n}=s^{2}=1, s r s^{-1}=r^{-1}$. In particular, let $n=2 m$. Then $x:=r^{m}$ generates the center $\mathbb{Z}_{2}=\langle x\rangle$ of $D_{2 n}$. Let us then gauge the center. Since the extension $0 \rightarrow \mathbb{Z}_{2} \rightarrow D_{2 n} \rightarrow D_{n} \rightarrow 0$ is nontrivial, the dual group $G^{\prime}=\hat{\mathbb{Z}}_{2} \times D_{n}$ has a nontrivial anomaly $\alpha_{\kappa_{D}}$, given in terms of a nonzero $\kappa_{D} \in H^{2}\left(D_{n}, \mathbb{Z}_{2}\right)$ describing the extension. In particular, for $D_{2 n}=D_{8}$, the dual group $G^{\prime}=\hat{\mathbb{Z}}_{2} \times \mathbb{Z}_{2} \times \mathbb{Z}_{2}$ is Abelian. Dually, this means that by gauging $\hat{\mathbb{Z}}_{2}$ of the Abelian group $G^{\prime}$ with an anomaly $\alpha_{\kappa_{D}}$ turns the symmetry into a non-Abelian group $D_{8}$.

As a final example in this subsection, consider $G=Q_{8}$, the quaternion group of eight elements, formed by eight quaternions $\pm 1, \pm i, \pm j, \pm k$. This is naturally a subgroup of $\mathrm{SU}(2)$ since quaternions of absolute value 1 form the group $\mathrm{SU}(2)$, and as such the lift to $\mathrm{SO}(3)$ of a finite subgroup of $\mathrm{SO}(3)$, this case $D_{4}=\mathbb{Z}_{2} \times \mathbb{Z}_{2}$. This means that we have a nontrivial extension

$$
0 \rightarrow \mathbb{Z}_{2} \rightarrow Q_{8} \rightarrow D_{4} \rightarrow 0
$$

This extension is again nontrivial, whose class $\kappa_{Q} \in H^{2}\left(\mathbb{Z}_{2} \times \mathbb{Z}_{2}, \mathbb{Z}_{2}\right)$ is different from $\kappa_{D}$ in the case of $D_{8}$. The dual group is then $G^{\prime}=\hat{\mathbb{Z}}_{2} \times \mathbb{Z}_{2} \times \mathbb{Z}_{2}$ but with a different anomaly $\alpha_{\kappa_{Q}}$.

Dually, we can say as follows. The same Abelian group, $\hat{\mathbb{Z}}_{2} \times \mathbb{Z}_{2} \times \mathbb{Z}_{2}$ with two different anomalies $\kappa_{D}$ and $\kappa_{Q}$ dualizes, under gauging of $\hat{\mathbb{Z}}_{2}$, into two different non-Abelian groups $D_{8}$ and $Q_{8}$.

\subsection{Integral symmetry categories of total dimension 6}

Let us study the symmetry categories of total dimension 6 in detail. We already know a few such symmetry categories, $\mathcal{C}\left(\mathbb{Z}_{2} \times \mathbb{Z}_{3}, \alpha\right), \mathcal{C}\left(S_{3}, \alpha\right)$ and $\operatorname{Rep}\left(S_{3}\right)$, where $S_{3}$ is the symmetric group acing on three objects. Let us study what the gauging of their subgroups leads to. We will see that there are in fact two more integral symmetry categories of total dimension 6 .

From $\mathcal{C}\left(\mathbb{Z}_{\mathbf{2}} \times \mathbb{Z}_{\mathbf{3}}, \boldsymbol{\alpha}\right)$. Here the anomaly is determined by $\alpha \in H^{3}\left(\mathbb{Z}_{2} \times \mathbb{Z}_{3}, \mathrm{U}(1)\right)=\mathbb{Z}_{2} \times \mathbb{Z}_{3}$.

- $\mathbb{Z}_{1}$ is always gaugeable,

- $\mathbb{Z}_{2}$ is gaugeable only when $\alpha$ is from $\mathbb{Z}_{3}$ and then the dual is itself,

- $\mathbb{Z}_{3}$ is gaugeable only when $\alpha$ is from $\mathbb{Z}_{2}$ and then the dual is itself,

- $\mathbb{Z}_{6}$ is gaugeable only when $\alpha$ is trivial.

So there is nothing particularly interesting going on here. 
From $\operatorname{Rep}\left(\boldsymbol{S}_{\mathbf{3}}\right)$. Any possible gauging of $\operatorname{Rep}\left(S_{3}\right)$ can always be done by first gauging $\operatorname{Rep}\left(S_{3}\right)$ back to $\mathcal{C}\left(S_{3}\right)$ and then gauge one of its subgroup. Therefore we do not have to study it separately.

From $\mathcal{C}\left(\boldsymbol{S}_{\mathbf{3}}, \boldsymbol{\alpha}\right)$. Here the anomaly is determined by $\alpha \in H^{3}\left(S_{3}, \mathrm{U}(1)\right)=\mathbb{Z}_{2} \times \mathbb{Z}_{3}$. Let us denote by $a$ and $b$ the generators of $\mathbb{Z}_{2}$ and of $\mathbb{Z}_{3}$, respectively.

- $\mathbb{Z}_{1}$ is always gaugeable and the dual is itself.

- The subgroup $\mathbb{Z}_{2}$ is gaugeable only when $\alpha=b^{i}$ with $i=0,1,2$. The dual is not of the form $\mathcal{C}\left(G^{\prime}, \alpha^{\prime}\right)$ because this subgroup is not normal. When $\alpha=0$ the dual turns out to be $\operatorname{Rep}\left(S_{3}\right)$. When $i=1,2$, the duals cannot be $\operatorname{Rep}\left(S_{3}\right)$, since if so, a further gauging will produce $\mathcal{C}\left(S_{3}, b^{0}\right)$ from $\mathcal{C}\left(S_{3}, b^{1,2}\right)$. But this is impossible, since these two symmetry categories have different number of possible gaugings.

- The normal subgroup $\mathbb{Z}_{3}$ is gaugeable only when $\alpha=a^{0,1}$. Gauging it leads back to itself, with the same anomaly.

- $S_{3}$ is gaugeable only when $\alpha$ is trivial. The dual is $\operatorname{Rep}\left(S_{3}\right)$.

From the analysis above, we find that symmetry categories $\mathcal{C}\left(S_{3}, b^{1,2} ; \mathbb{Z}_{2}, 0\right)$ obtained by gauging the $\mathbb{Z}_{2}$ subgroup of $S_{3}$ with a nontrivial anomaly $\alpha=b^{1,2}$ is neither of the form $\mathcal{C}(G, \alpha)$ nor of the form $\operatorname{Rep}\left(S_{3}\right)$. It turns out that they have the same fusion rule as $\operatorname{Rep}\left(S_{3}\right)$, namely there are simple objects $1, x$ of dimension 1 and $a$ of dimension 2 , such that $x^{2}=1, a x=a, a^{2}=1+x+a$. These are the smallest integral symmetry categories which is neither $\mathcal{C}(G, \alpha)$ nor $\operatorname{Rep}(G)$.

It is known that the symmetry categories we listed so far exhaust all possible integral symmetry categories of dimension 6 . This was shown in [32].

\subsection{Integral symmetry categories of total dimension 8}

Let us next have a look at symmetry categories of dimension 8 . There are five finite groups $G$ of order 8 , namely the three Abelian ones $\mathbb{Z}_{8}, \mathbb{Z}_{2} \times \mathbb{Z}_{4},\left(\mathbb{Z}_{2}\right)^{3}$ and two non-Abelian ones $D_{8}$ and $Q_{8}$. Correspondingly, we already see that there are symmetry categories $\mathcal{C}(G, \alpha)$ constructed from these group, where the possible anomalies are given as follows:

\begin{tabular}{|c|c|c|c|c|c|}
\hline$G$ & $\mathbb{Z}_{8}$ & $\mathbb{Z}_{2} \times \mathbb{Z}_{4}$ & $\mathbb{Z}_{2}^{3}$ & $D_{8}$ & $Q_{8}$ \\
\hline$H^{3}(G, \mathrm{U}(1))$ & $\mathbb{Z}_{8}$ & $\mathbb{Z}_{2}^{2} \times \mathbb{Z}_{4}$ & $\mathbb{Z}_{2}^{7}$ & $\mathbb{Z}_{2}^{2} \times \mathbb{Z}_{4}$ & $\mathbb{Z}_{8}$ \\
\hline
\end{tabular}

We also know two other symmetry categories of total dimension 8 , namely the representation categories $\operatorname{Rep}\left(D_{8}\right)$ and $\operatorname{Rep}\left(Q_{8}\right)$.

These two representation categories have the same fusion rules: there are four dimension-1 simple objects $1, a, b, a b$ forming an Abelian group $\mathcal{A}=\mathbb{Z}_{2} \times \mathbb{Z}_{2}$, and one dimension-2 simple object $m$, such that the fusion rule is commutative, $a \otimes m=b \otimes m=m$, and

$$
m \otimes m=1 \oplus a \oplus b \oplus a b .
$$


There are in fact two more symmetry categories, known as KP and TY with this fusion rule [15]. For all these four cases, it is known that we can gauge the subsymmetry $\mathbb{Z}_{2}=$ $\{1, a\}$ and obtain $\mathcal{C}\left(D_{8}, \alpha\right)$ where $\alpha \in H^{3}\left(D_{8}, \mathrm{U}(1)\right)$ is chosen depending on the four cases.

The four symmetry categories with the above fusion rule have a nice uniform description due to Tambara and Yamagami, which is applicable to a more general case based on any Abelian group $\mathcal{A}$. The gauging of $\mathbb{Z}_{2}=\{1, a\}$ leading to $\mathcal{C}\left(D_{8}, \alpha\right)$ also has an explanation in the larger context of Tambara-Yamagami categories. We will study them in more detail in the next subection.

The only remaining choice of the fusion rule of an integral symmetry category of total dimension 8 has the following form [33]: there are four dimension-1 simple objects $1, c$, $c^{2}, c^{3}$ forming an Abelian group $\mathcal{A}=\mathbb{Z}_{4}$, and one dimension-2 simple object $m$, such that $c \otimes m=m \otimes c=m$ and

$$
m \otimes m=1 \oplus c \oplus c^{2} \oplus c^{3} .
$$

The result of Tambara and Yamagami [15] implies that there are four symmetry categories with this fusion rule, distinguished by two sign choices. These categories do not have a common name; let us temporarily call it $\mathrm{S}_{ \pm \pm}$. This completes the list of the integral symmetry category of total dimension 8 .

Before moving on, we have two comments. First, every integral symmetry categories we saw so far, i.e. those symmetry categories for which dimensions of objects are integers, can be obtained by gauging a non-anomalous subgroup of a possibly anomalous group. This property fails when the total dimension is larger. Indeed, some of the Tambara-Yamagami categories we discuss next are integral but cannot be obtained by gauging a non-anomalous subgroup of a possibly anomalous group.

Second, the symmetry category KP is of a historical interest, since it is the category of representations of the first non-commutative non-cocommutative Hopf algebra that appeared in the literature, constructed by Kac and Paljutkin in 1966 [34]. One way to construct a symmetry category is to pick a Hopf algebra $H$ and take the category of its representations. When $H$ is commutative, the symmetry category is of the form $\mathcal{C}(G)$, and when $H$ is cocommutative, the symmetry category is of the form $\operatorname{Rep}(G)$. When we take the dual of a Hopf algebra, this naturally interchanges $\mathcal{C}(G)$ and $\operatorname{Rep}(G)$. Therefore considering Hopf algebras is a unified framework in which $\mathcal{C}(G)$ and $\operatorname{Rep}(G)$ can be treated symmetrically. That said, to treat the symmetries of two dimensional theories and their gauging, we need to deal with symmetry categories in general and we cannot stop at the level of the Hopf algebras. The symmetry categories which are categories of representations of Hopf algebras can be characterized as symmetry categories which has Vec as a module category. But even the familiar $\mathcal{C}(G, \alpha)$ with a nontrivial $\alpha$ does not have Vec as a module category!

\subsection{Tambara-Yamagami categories}

To construct a Tambara-Yamagami category, we start from an Abelian group $\mathcal{A}$. The simple objects of the category are elements $a \in \mathcal{A}$ of dimension 1 together with an object 
$m$ of dimension $|\mathcal{A}|$, with the commutative fusion rule

$$
a \otimes m=m, \quad m \otimes m=\bigoplus_{a \in \mathcal{A}} a .
$$

Tambara and Yamagami showed in [15] that any symmetry category with this fusion ring is given by the choice of a symmetric nondegenerate bicharacter $\chi: \mathcal{A} \times \mathcal{A} \rightarrow \mathrm{U}(1)$ and the choice of the sign of $\tau= \pm 1 / \sqrt{|\mathcal{A}|}$. The nontrivial associators are given in terms of $\chi$ and $\tau$ :

$$
\begin{aligned}
a_{a, m, b} & =\chi(a, b), \\
a_{m, a, m} & =\bigoplus_{b} \chi(a, b) \operatorname{id}_{b}, \\
a_{m, m, m} & =\tau\left(\chi(a, b)^{-1}\right)_{a, b} \in \operatorname{Hom}\left(\bigoplus_{a} m, \bigoplus_{b} m\right) .
\end{aligned}
$$

Let us denote the resulting symmetry category by $\operatorname{TY}(\mathcal{A}, \chi, \tau)$.

In our case where $\mathcal{A}=\mathbb{Z}_{2} \times \mathbb{Z}_{2}$ generated by $a$ and $b$, we just have two possible symmetric nondegenerate bicharacters $\chi$ up to the action of $\operatorname{SL}\left(2, \mathbb{Z}_{2}\right)$. Explicitly, two such choices are specified by

$$
\chi(a, a)=1, \quad \chi(b, b)=-\chi(a, b)= \pm 1 .
$$

We denote the choices by $\chi_{ \pm}$. The choice of $\tau$ is $\tau= \pm 1 / 2$. Then we have the following correspondence:

$$
\begin{array}{|c|cc|}
\hline & \chi_{1} & \tau \\
\hline \operatorname{Rep}\left(D_{8}\right) & \chi_{+} & +1 / 2 \\
\operatorname{Rep}\left(Q_{8}\right) & \chi_{+} & -1 / 2 \\
\mathrm{KP} & \chi_{-} & +1 / 2 \\
\mathrm{TY} & \chi_{-} & -1 / 2 \\
\hline
\end{array}
$$

Here we are slightly abusing the notation such that TY alone stands for a specific symmetry category with total dimension 8 , while $\operatorname{TY}(\mathcal{A}, \chi, \tau)$ refers to a general construction.

Another Tambara-Yamagami category of total dimension 8 is based on $\mathcal{A}=\mathbb{Z}_{4}=$ $\left\{1, c, c^{2}, c^{3}\right\}$. Any non-degenerate symmetric bicharacter is of the form

$$
\chi_{ \pm}\left(c^{k}, c^{l}\right)=( \pm i)^{k l} .
$$

Together with the choice of the sign of $\tau$, we have four Tambara-Yamagami categories $\mathrm{S}_{ \pm \pm}$ based on $\mathbb{Z}_{4}$. We note that the bicharacter $\chi_{+}$is trivial on the subgroup $\mathbb{Z}_{2}=\left\{1, a=c^{2}\right\}$.

It is known that for all eight Tambara-Yamagami categories described above, we can gauge the subsymmetry $\mathbb{Z}_{2}=\{1, a\}$ and obtain $\mathcal{C}\left(D_{8}, \alpha\right)$ where $\alpha \in H^{3}\left(D_{8}, \mathrm{U}(1)\right)$ is chosen depending on the four cases. This fact is a specific instance of a general theorem determining when a Tambara-Yamagami category is obtained by gauging a subgroup of a possibly non-anomalous group [35]. They showed that this occurs if and only if $\mathcal{A}$ has a Lagrangian subgroup $H$ for $\chi$, i.e. there is a subgroup $H \subset \mathcal{A}$ such that i) the restriction of 
$\chi$ on $H$ is trivial and ii) $\mathcal{A} / H \simeq \hat{H}$ via the pairing induced by $\chi$. In the four cases above, $H$ is given by $\mathbb{Z}_{2}=\{1, a\}$.

Let us explicitly describe below that gauging the subgroup $H$ of the Tambara-Yamagami symmetry category $\mathcal{C}=\operatorname{TY}(\mathcal{A}, \chi, \tau)$ produces a symmetry category of the form $\mathcal{C}(G, \alpha)$. The algebra object we use to gauge the system is $A=\bigoplus_{h \in H} h$.

We note that $\mathcal{A}$ fits in the extension $0 \rightarrow H \rightarrow \mathcal{A} \rightarrow \hat{H} \rightarrow 0$. We fix a specific section $s: \hat{H} \rightarrow \mathcal{A}$ and denote by $\kappa$ the two-cocycle in $C^{2}(\hat{H}, H)$ characterizing this extension. Then the symmetric nondegenerate bicharacter $\chi$ on $\mathcal{A}$ defines a symmetric map

$$
\chi: \hat{H} \times \hat{H} \rightarrow \mathrm{U}(1)
$$

satisfying the condition

$$
\chi\left(\rho+\rho^{\prime}, \sigma\right)=\chi(\rho, \sigma)+\chi\left(\rho^{\prime}, \sigma\right)+\sigma\left(\kappa\left(\rho, \rho^{\prime}\right)\right) .
$$

Simple $(A, A)$ bimodules turn out to be isomorphic to either of the following two types:

- $X_{\rho, \sigma}$ for $\rho, \sigma \in \hat{H}$. As an object in $\mathcal{C}$, it is $\bigoplus_{h \in H} h \sigma$. The right action of $A$ is trivial, and the left action of $A$ is given by $\rho(a)$ id $: a \otimes h \sigma \rightarrow a h \sigma$.

- $Y_{\rho, \sigma}$ for $\rho, \sigma \in \hat{H}$. As an object in $\mathcal{C}$, it is just $m$. The left action and the right action of $A$ is given by $\rho(a)$ id $: a \otimes m \rightarrow m$ and $\sigma(a)$ id $: m \otimes a \rightarrow m$.

The tensor product $\otimes_{A}$ in the category of bimodules, together with the projections $\pi$ (4.5) in the definition of $\otimes_{A}$ is then given as follows:

$$
X_{\rho, \sigma} \otimes_{A} X_{\rho^{\prime}, \sigma^{\prime}}=X_{\rho \rho^{\prime}, \sigma \sigma^{\prime}}
$$

where the projections $\pi$ are trivial,

$$
X_{\rho, \sigma} \otimes_{A} Y_{\rho^{\prime}, \sigma^{\prime}}=Y_{\rho \rho^{\prime}, \sigma \sigma^{\prime}}
$$

where the projections $\pi$ are given by $\rho^{\prime}(a)$ id $: a \sigma \otimes m \rightarrow m$,

$$
Y_{\rho, \sigma} \otimes_{A} X_{\rho^{\prime}, \sigma^{\prime}}=Y_{\rho\left(\sigma^{\prime}\right)^{-1}, \sigma\left(\rho^{\prime}\right)^{-1}}
$$

where the projections $\pi$ are given by $\sigma(a) \rho^{\prime}(a)^{-1}$ id $: m \otimes a \sigma^{\prime} \rightarrow m$, and

$$
Y_{\rho, \sigma} \otimes_{A} Y_{\rho^{\prime}, \sigma^{\prime}}=X_{\rho\left(\sigma^{\prime}\right)^{-1}, \sigma\left(\rho^{\prime}\right)^{-1}}
$$

where the projections $\pi$ are given by

$$
\pi=\bigoplus_{a \in H} \sigma^{\prime}(a)^{-1} \mathrm{id}_{a \sigma\left(\rho^{\prime}\right)^{-1}}: m \otimes m \rightarrow \bigoplus_{x \in \mathcal{A}} x .
$$

The equations (5.30), (5.31), (5.32), (5.33) show that the simple objects $X_{\rho, \sigma}$ and $Y_{\rho, \sigma}$ form the group

$$
G=(\hat{H} \times \hat{H}) \rtimes \mathbb{Z}_{2}
$$


where the $\mathbb{Z}_{2}$ acts by

$$
(\rho, \sigma) \mapsto(-\sigma,-\rho) .
$$

The anomaly $\alpha$ can be computed using the projections given above and the associators (5.22), (5.23) and (5.24) of the original category. We find

$$
\begin{aligned}
\alpha\left(X_{\rho, \sigma}, X_{\rho^{\prime}, \sigma^{\prime}}, X_{\rho^{\prime \prime}, \sigma^{\prime \prime}}\right) & =\rho^{\prime \prime}\left(\kappa\left(\sigma, \sigma^{\prime}\right)\right), \\
\alpha\left(X_{\rho, \sigma}, X_{\rho^{\prime}, \sigma^{\prime}}, Y_{\rho^{\prime \prime}, \sigma^{\prime \prime}}\right) & =\rho^{\prime \prime}\left(\kappa\left(\sigma, \sigma^{\prime}\right)\right), \\
\alpha\left(X_{\rho, \sigma}, Y_{\rho^{\prime}, \sigma^{\prime}}, X_{\rho^{\prime \prime}, \sigma^{\prime \prime}}\right) & =\chi\left(\sigma, \sigma^{\prime \prime}\right), \\
\alpha\left(Y_{\rho, \sigma}, X_{\rho^{\prime}, \sigma^{\prime}}, X_{\rho^{\prime \prime}, \sigma^{\prime \prime}}\right) & =\left(\rho^{\prime} \rho^{\prime \prime} \sigma^{-1}\right)\left(\kappa\left(\sigma^{\prime}, \sigma^{\prime \prime}\right)\right), \\
\alpha\left(X_{\rho, \sigma}, Y_{\rho^{\prime}, \sigma^{\prime}}, Y_{\rho^{\prime \prime}, \sigma^{\prime \prime}}\right) & =\sigma^{\prime \prime}\left(\kappa\left(\sigma,\left(\sigma^{\prime}\right)^{-1} \rho^{\prime \prime}\right)\right), \\
\alpha\left(Y_{\rho, \sigma}, X_{\rho^{\prime}, \sigma^{\prime}}, Y_{\rho^{\prime \prime}, \sigma^{\prime \prime}}\right) & =\chi\left(\sigma^{\prime}, \sigma\left(\rho^{\prime}\right)^{-1}\left(\rho^{\prime \prime}\right)^{-1}\right), \\
\alpha\left(Y_{\rho, \sigma}, Y_{\rho^{\prime}, \sigma^{\prime}}, X_{\rho^{\prime \prime}, \sigma^{\prime \prime}}\right) & =\left(\sigma^{\prime}\left(\rho^{\prime \prime}\right)^{-1}\right)\left(\kappa\left(\sigma\left(\rho^{\prime}\right)^{-1}, \sigma^{\prime \prime}\right)\right), \\
\alpha\left(Y_{\rho, \sigma}, Y_{\rho^{\prime}, \sigma^{\prime}}, Y_{\rho^{\prime \prime}, \sigma^{\prime \prime}}\right) & =\operatorname{sgn}(\tau) \chi\left(\sigma\left(\rho^{\prime}\right)^{-1}, \sigma^{\prime}\left(\rho^{\prime \prime}\right)^{-1}\right) .
\end{aligned}
$$

As a check of the computation, we can directly confirm that these define a 3-cocycle on $G$.

In the eight cases $\operatorname{Rep}\left(D_{8}\right), \operatorname{Rep}\left(Q_{8}\right), \mathrm{KP}, \mathrm{TY}$ and $\mathrm{S}_{ \pm \pm}$we discussed above, we always have $H=\mathbb{Z}_{2}$ and the resulting group $G=\left(\mathbb{Z}_{2} \times \mathbb{Z}_{2}\right) \rtimes \mathbb{Z}_{2}$ is $D_{8}$. To see this, regard $\mathbb{Z}_{2} \times \mathbb{Z}_{2}$ as the group of flipping the coordinates $x$ and $y$ of $\mathbb{R}^{2}$ generated by

$$
(x, y) \mapsto(-x, y), \quad(x, y) \mapsto(x,-y)
$$

respectively, and $\mathbb{Z}_{2}$ acting on $\mathbb{Z}_{2} \times \mathbb{Z}_{2}$ to be the exchange of $x$ and $y$ given by

$$
(x, y) \mapsto(y, x) .
$$

Dually, with a suitably chosen $\alpha$ on $D_{8}$ and gauging the $\mathbb{Z}_{2}$ subgroup flipping the $x$ coordinate, we get the four symmetry categories given above.

\section{$6 \quad$ 2d TFT with $\mathcal{C}$ symmetry and their gauging}

\subsection{2d TFTs without symmetry}

As a warm-up, let us recall the structure of $2 \mathrm{~d}$ TFTs without any symmetry. We follow the exposition in [10] closely, see in particular their appendix A.

We start with a vector space $V$ of states on $S^{1}$ and one wants to define a consistent transition amplitude

$$
Z_{\Sigma}: V^{\otimes m} \rightarrow V^{\otimes n}
$$

corresponding to a given topological surface $\Sigma$ with $m$ incoming circles and $n$ outgoing circles. We need four basic maps ${ }^{\circ} I, I, M,{ }^{\circ} M$ corresponding to four basic geometries given in figure 25.

First, we construct maps ${ }^{\circ} I M: V \otimes V \rightarrow \mathbb{C}$ and ${ }^{\circ} M I: \mathbb{C} \rightarrow V \otimes V$ as in figure 26. This inner product must be non-degenerate because it just corresponds to a cylinder geometry which pairs a state on one circle with a dual state on the other circle. Using it, we can 


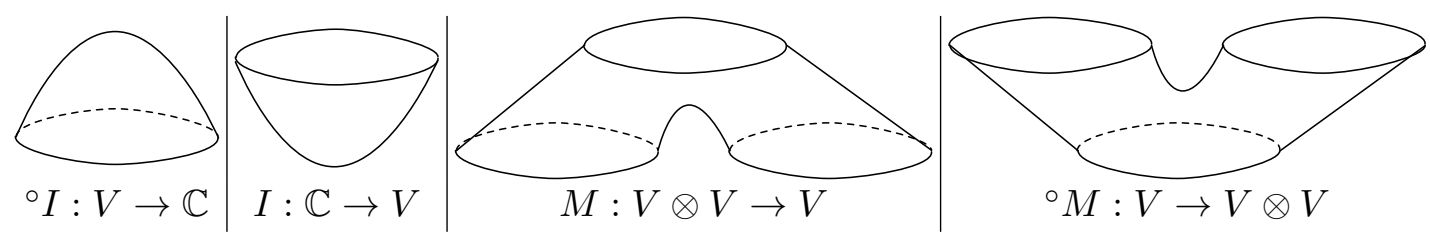

Figure 25. The basic building blocks of a $2 \mathrm{~d}$ TFT.

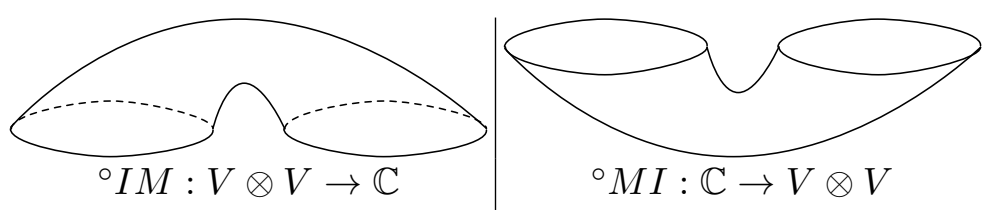

Figure 26. The pairing of $V$ with itself.

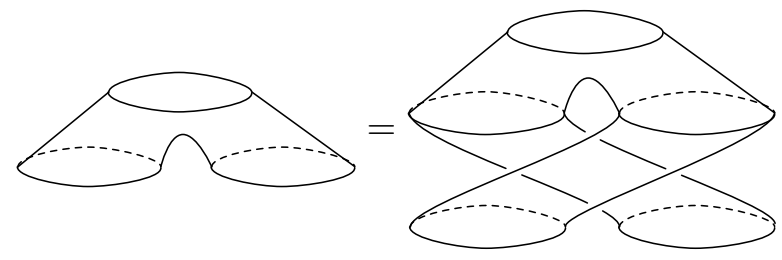

Figure 27. The product $M$ is commutative.

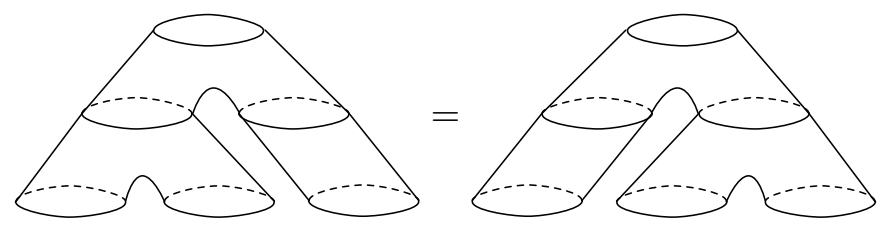

Figure 28. The product $M$ is associative.

identify $V$ and $V^{*}$. Then, ${ }^{\circ} I$ is an adjoint of $I$ and ${ }^{\circ} M$ is an adjoint of $M$. Therefore, to every property involving $M$, we can write down a corresponding property involving ${ }^{\circ} M$, and similarly for statements about $I$ and ${ }^{\circ} I$. This allows us to reduce the number of independent statements we need to write down roughly by half; we do not repeat these adjoint statements below.

We consider $M$ as giving a product on $V$. There is no order on the two incoming circles of a pair of pants and hence the product is commutative, see figure 27 . We can also see that $M$ is associative from figure 28 and that $I$ is a unit of the multiplication $M$ from figure 29. Also, by composing these inner products with the product, we see that the product is invariant under permuting three legs, see figure 30.

After these preparations, let us associate $a$ map $Z_{\Sigma}: V^{\otimes m} \rightarrow V^{\otimes n}$ to a surface $\Sigma$ with $m$ incoming circles and $n$ outgoing circles. We pick a time coordinate $t: \Sigma \rightarrow[0,1]$ such that at $t=0$ we start with $m$ initial circles and at $t=1$ we finish with $n$ final circles. As time goes from 0 to 1 , the number of circles generically stay constant but can either 


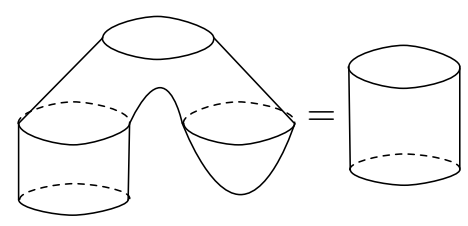

Figure 29. $I$ is a unit of the product $M$.

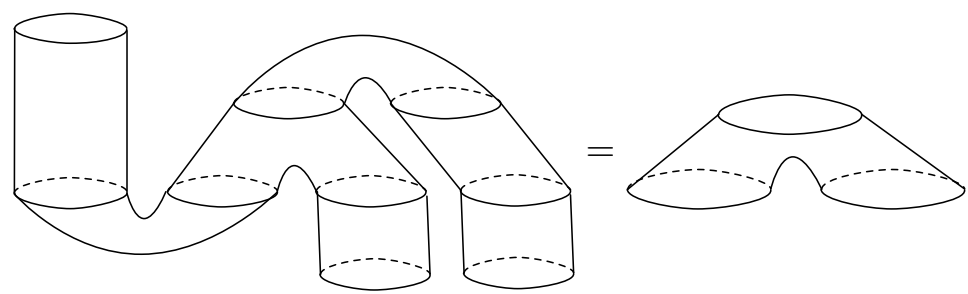

Figure 30. The product is invariant under exchanging an incoming circle and an outgoing circle.

increase or decrease by one unit at specific times $0=t_{0}<t_{1}<t_{2}<\cdots<t_{p}=1$. Cut $\Sigma$ once in each interval $\left(t_{i}, t_{i+1}\right)$. This divides $\Sigma$ into $p$ pieces. The geometry of each piece contains some cylinders, which correspond to trivial transition amplitude, and exactly one non-trivial geometry out of the four non-trivial cases shown in figure 25 . This gives us an expression for $Z_{\Sigma}$ in terms of the four maps ${ }^{\circ} I, I, M,{ }^{\circ} M$.

However, one could choose a different time $t^{\prime}$ which starts with same $m$ initial circles and ends with same $n$ final circles. In general, this would lead to a different cutting of $\Sigma$ and a different compositions of four maps ${ }^{\circ} I, I, M,{ }^{\circ} M$. We need to make sure that they agree.

We can continuously deform the time function $t$ to obtain the time function $t^{\prime}$. The critical points $t_{i}$ will move under this deformation and will cross each other. It is also possible for two critical points to meet and annihilate each other or for two critical points to pop out of nowhere. We therefore need to ensure that $Z_{\Sigma}$ remains invariant when $t_{i}$ and $t_{i+1}$ cross each other, and when two critical points are created or annihilated. For this, we just need to ensure that the two-step composition from the cut between $t_{i-1}$ and $t_{i}$ to the cut between $t_{i+1}$ and $t_{i+2}$ remains invariant under these processes.

All possible types of the topology changes were enumerated carefully in appendix A of [10]. The cases are the following and their adjoints:

1. The creation or the annihilation of two critical points as shown in figure 29 , or

2a. The exchange of two critical points as shown in figure 28 , which we already encountered, or

2b. the situation figure 31 where the number of intermediate circles changes from one to three, or

2c. the situation figure 32 where the A-cycle and the B-cycle of a torus is exchanged. In more detail, on one side, a circle consisting of segments $a, b, c, d$ in this order splits to two circles consisting of $a, b$ and $c, d$, which are now along the A-cycle. They then 


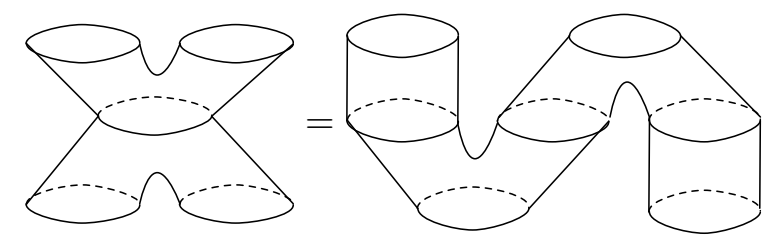

Figure 31. One possible topology change.
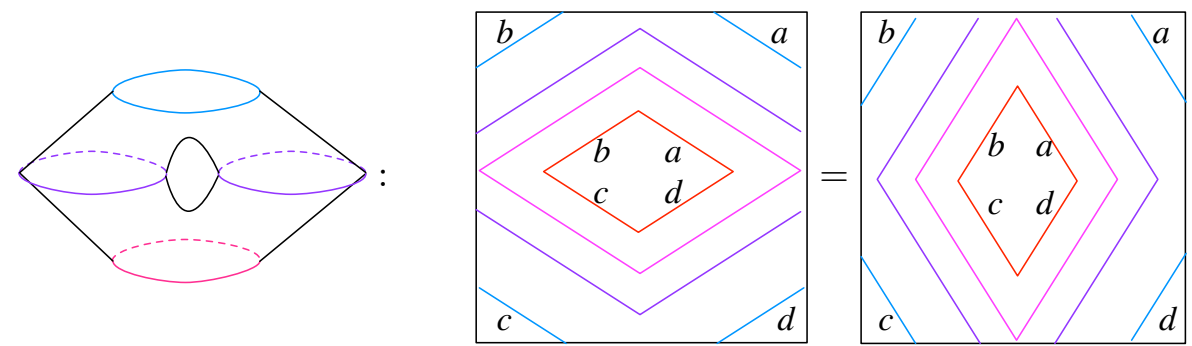

Figure 32. Another possible topology change concerns a torus with two holes. On the two figures on the right, the time flows from inside to the outside, and the parallel edges of the boundary need to be identified to form a torus. On one side, the intermediate two circles are along the A-cycle, and on the other side, they are along the B-cycle.

merge into a circle consisting of four segments with the order $b, a, d, c$. On the other side, the two circles in the intermediate stage consists of segments $b, c$ and $d, a$, and are along the B-cycle.

The invariance of $Z_{\Sigma}$ under the change 1 is the unit property itself, and the invariance under the change $2 \mathrm{a}$ is the associativity itself. The invariance under the change $2 \mathrm{~b}$ can be reduced to associativity by using the cyclic invariance of the product, shown in figure 30 . Finally, under the topology change $3 \mathrm{~b}$, the map $Z_{\Sigma}$ is trivially invariant.

In total, we have shown that a $2 \mathrm{~d}$ TFT with no symmetry is completely defined by a vector space $V$ with the four maps ${ }^{\circ} I, I, M,{ }^{\circ} M$ with the conditions described above. Such a vector space is known as a commutative Frobenius algebra $V$.

\subsection{TFT with $\mathcal{C}$ symmetry on a cylinder}

Let us now move on to the discussion of TFTs with symmetry given by a symmetry category $\mathcal{C}$. In this subsection we start with the simplest geometry, namely cylinders. We already discussed basics in section 4.8.1. As mentioned there, we choose a base point along each constant-time cicle, and call its trajectory the auxiliary line.

Basic ingredients. We first associate the Hibert space $V_{a}$ for a circle with a single insertion of a line labeled by $a \in \mathcal{C}$. We require $V_{a \oplus b}=V_{a} \oplus V_{b}$. We now associate a Hilbert space $V_{a, b, c, \ldots}$ for a circle with insertions of transverse lines $a, b, c, \ldots$ by fusing them in a fixed particular order, starting from the closest line on the right of the base point and then toward the right:

$$
V_{a, b, c, \ldots}:=V_{(\cdots((a \otimes b) \otimes c) \cdots)} .
$$

The case with three lines is shown in figure 33. 


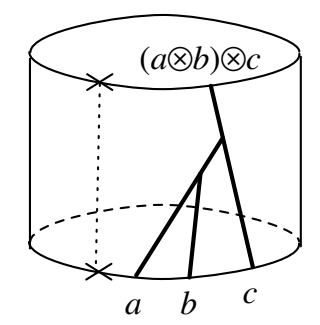

Figure 33. The Hilbert space of a circle with multiple line operators are identified with the Hilbert space of a circle with the fused line operator.

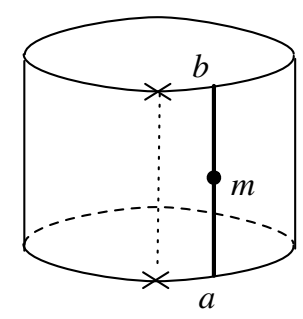

$Z(m): V_{a} \rightarrow V_{b}$

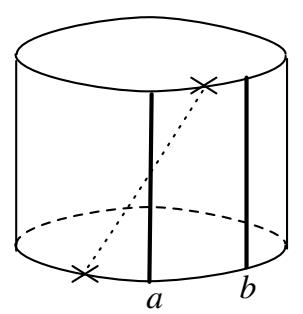

$X_{a, b}: V_{a \otimes b} \rightarrow V_{b \otimes a}$

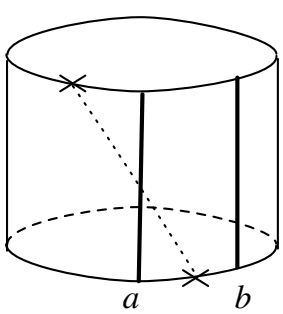

$Y_{b, a}: V_{b \otimes a} \rightarrow V_{a \otimes b}$

Figure 34. Basic operations on the cylinder.

We have two basic operations we can perform on the cylinder, see figure 34 . One is to insert a morphism $m: a \rightarrow b$, which defines an operator $Z(m): V_{a} \rightarrow V_{b}$. Another is to move the base point to the right and to the left, which defines morphisms $X_{a, b}: V_{a \otimes b} \rightarrow V_{b \otimes a}$ and $Y_{b, a}: V_{b \otimes a} \rightarrow V_{a \otimes b}$.

Assignment of a map to a given network. With these basic operations, we can assign $a$ map $V_{a, b, \ldots} \rightarrow V_{c, d, \ldots}$ for a cylinder equipped with an arbitrary network of lines and morphisms from the symmetry category $\mathcal{C}$, where an incoming circle have insertions $a, b, \ldots$ and an outgoing circle have insertions $c, d, \ldots$

We choose a time function $t$ on it, and we call any time $t_{i}$ a critical point when either of the following happens: i) there is an insertion of a morphism on a line, ii) there is a fusion of two lines $a, b$ into one line $a \otimes b$ or vice versa, and iii) a line crosses an auxiliary line. Note that we do not allow the auxiliary line to bend backward in time, as part of the definition.

We order $0=t_{0}<t_{1}<\cdots<t_{p-1}<t_{p}=1$ so that the incoming circle is at $t=0$ and the outgoing circle is at $t=1$. Each critical point of type i) gives a factor of $Z(m)$, that of type ii) gives a factor of $Z(\alpha)$ where $\alpha$ is an appropriate associator, and that of type iii) gives a factor of $X$ or $Y$. Then we define the map $V_{a, b, \cdots} \rightarrow V_{c, d, \cdots}$ associated with this time function $t$ to be the composition of factors corresponding to these critical points.

Consistency of the assignment. We now need to show that this assignment is consistent. There are three types of changes under which the assignment needs to be constant, namely

- the change of the time function $t$, 


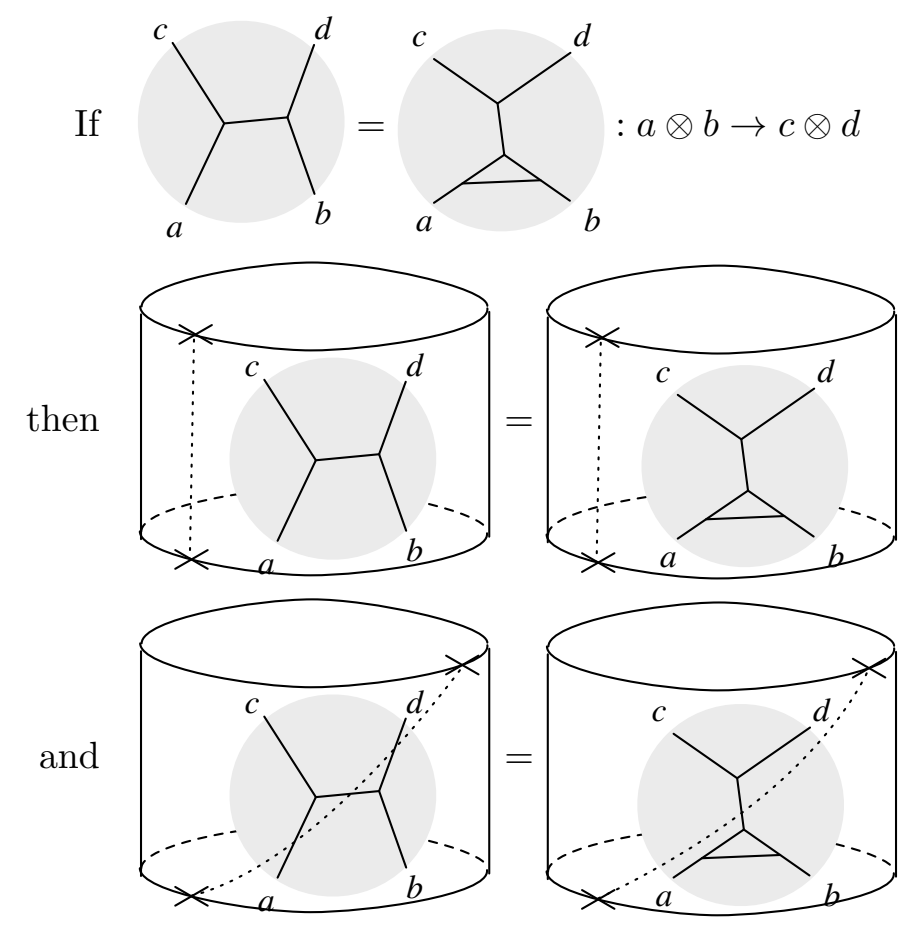

Figure 35. A local change in the network should not affect the map on the Hilbert space if the two subnetworks give the same morphism.

- the change of the positions of the auxiliary line, and

- the change of the network in a disk region that does not change the morphism within it.

The third point might need some clarification. In the symmetry category $\mathcal{C}$, a topologically different network can correspond to the same morphism. Then we need to ensure that if we replace a subnetwork on a cylinder accordingly, the resulting map on the Hilbert space should also be the same, see figure 35 This is not just a change in the time function, therefore we need to guarantee the invariance separately.

The auxiliary line might cut though the subdiagram, as also shown in figure 35, but this does not have to be treated separately, since we can first move the auxiliary line outside of the disk region, assuming that it is shown that the auxiliary lines can be moved.

Then this third type of change can be just taken care of by assuming that we can fuse two local operators, leading to the following constraint, see figure 36 :

$$
Z(n) Z(m)=Z(n \circ m) .
$$

Next, let us take care of the second type of change, where we move the auxiliary lines keeping the network and the time function fixed. First, moving the auxiliary line back and forth in succession should not do anything, so we have

$$
X_{a, b}=Y_{b, a}^{-1}
$$




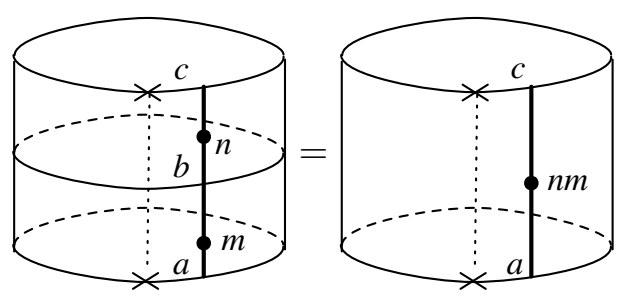

Figure 36. Two morphisms can be combined.

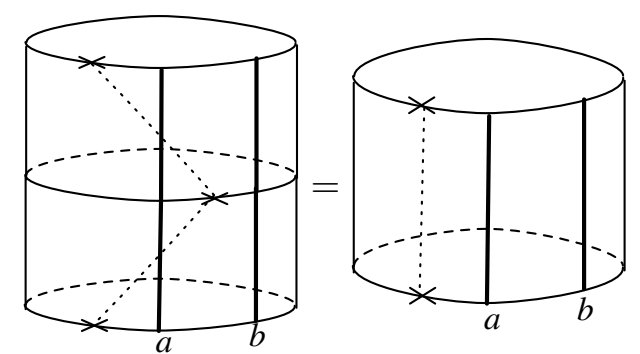

Figure 37. Moving the auxiliary line back and forth should do nothing.

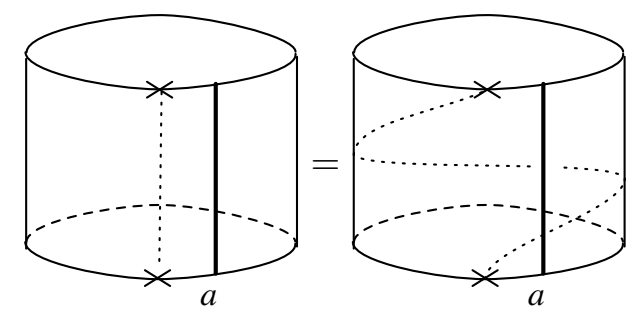

Figure 38. Winding the auxiliary line all the way around, represented by $X_{a, 1}$, should not do anything.

see figure 37. Rotating the base point all the way around should not do anything either, therefore we have

$$
X_{a, 1}=\mathrm{id}
$$

see figure 38 .

Then we should be able to move the morphisms across the auxiliary line, leading to two relations, as illustrated in figure 39:

$$
\begin{gathered}
X_{a^{\prime}, b} Z(m \otimes 1)=Z(1 \otimes m) X_{a, b}, \\
X_{a, b^{\prime}} Z(1 \otimes n)=Z(n \otimes 1) X_{a, b}
\end{gathered}
$$

for $m: a \rightarrow a^{\prime}$ and $n: b \rightarrow b^{\prime}$. We can also fuse two lines before crossing the auxiliary line, see figure 40. This leads to the constraint

$$
X_{b, c \otimes a} Z\left(\alpha_{b, c, a}\right) X_{a, b \otimes c} Z\left(\alpha_{a, b, c}\right)=Z\left(\alpha_{c, a, b}{ }^{-1}\right) X_{a \otimes b, c}
$$

Finally, on the cylinder, the change in the time function itself does not do much, and possible changes are already all covered. Thus, we see that to define a consistent TFT 

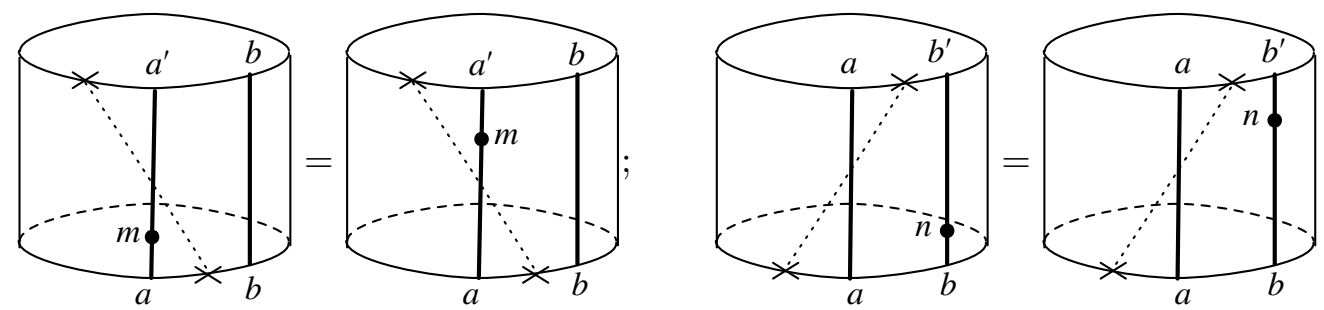

Figure 39. Morphisms can be crossed across the auxiliary line.

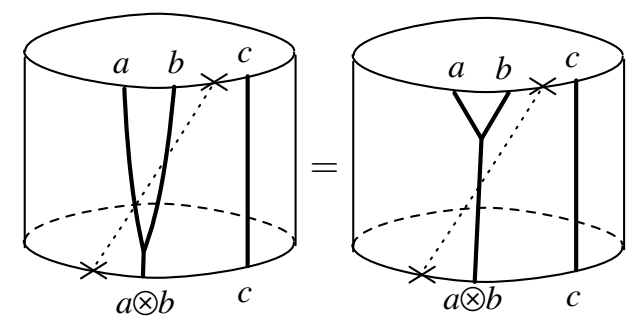

Figure 40. Crossing the fused line over the auxiliary line should be the same with crossing two lines separately.

with $\mathcal{C}$ symmetry on a cylinder, we need the data of an additive functor $Z: \mathcal{C} \rightarrow$ Vec with morphisms $X_{a, b}: V_{a \otimes b} \simeq V_{b \otimes a}$ satisfying (6.5), (6.6), (6.7) and (6.8).

Generalized associators on the cylinder. The relations so far guarantees that we can always move the base point and change the order of the tensoring of lines in a consistent manner. For example, the relation (6.8) means that there is a single well-defined isomorphism between $V_{(a \otimes b) \otimes c}$ and $V_{(c \otimes a) \otimes b}$. We introduce a notation

$$
\mathcal{A}_{(a \otimes b) \otimes c \rightarrow(c \otimes a) \otimes b}: V_{(a \otimes b) \otimes c} \rightarrow V_{(c \otimes a) \otimes b}
$$

for it, and call it a generalized associator on the cylinder. We similarly introduce generalized associators for an arbitrary motion of the base point and an arbitrary rearrangement of parentheses. Each such generalized associator have multiple distinct-looking expressions in terms of sequences of $Z(\alpha), X$ and $X^{-1}$, but they give rise to the same isomorphism.

\subsection{TFT with $\mathcal{C}$ symmetry on a general geometry}

Basic data. Let us discuss now the TFT with $\mathcal{C}$ symmetry on a general geometry. The four basic geometries are given in figure 41. For a pair of pants, we need to join the two auxiliary lines coming from each leg into a single auxiliary line. We take the point where this happens to coincide with the critical point where two circles join to form a single circle. In what follows, we will refer to the initial two legs of a pair of pants as the initial legs and the final leg as the product leg.

We can now associate to any geometry $\Sigma$ with $m$ initial legs and $n$ final legs with an arbitrarily complicated network of lines and morphisms from $\mathcal{C}$ a linear map as follows. We first choose a time function $t: \Sigma \rightarrow[0,1]$. We call a time value $t_{i}$ critical when any 


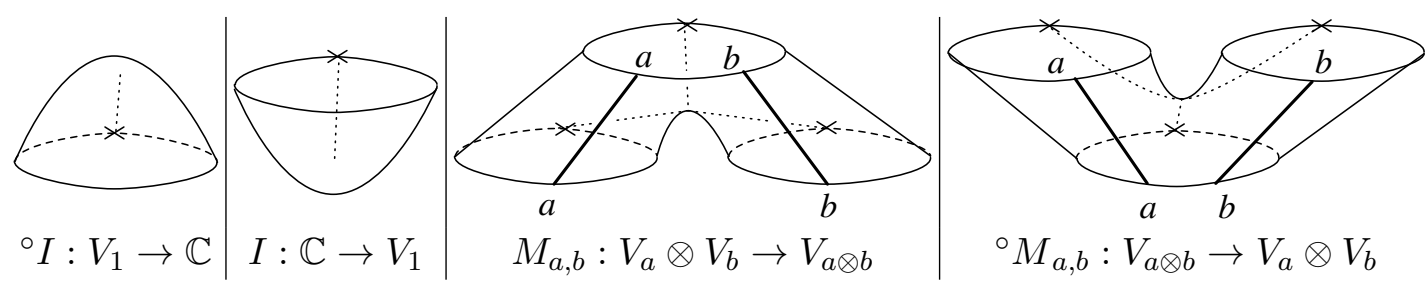

Figure 41. The basic building blocks of a $2 \mathrm{~d}$ TFT with $\mathcal{C}$ symmetry.

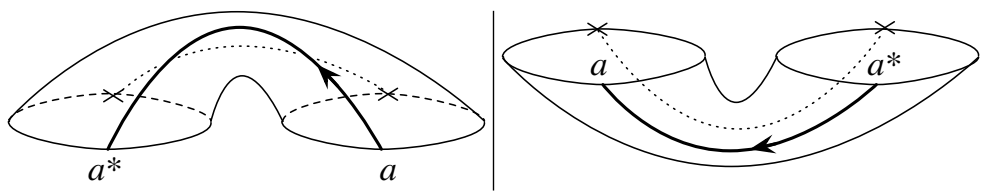

Figure 42. The pairing of $V_{a}$ and $V_{a^{*}}$.

of the following happens: i) the topology of the constant time slice change, ii) there is a morphism, or iii) a line crosses a auxiliary line. We order the critical times so that $0=t_{0}<t_{1}<t_{2}<\cdots<t_{p}=1$. We cut $\Sigma$ once in each interval $\left(t_{i}, t_{i+1}\right)$, and associate to each critical time $t_{i}$ one of the basic linear maps. We then compose them. We now need to guarantee that this assignment is consistent.

Basic consistency conditions. Let us first enumerate basic consistency conditions. First, we define the pairing of $V_{a}$ and $V_{a^{*}}$ as in figure 42 :

$$
\begin{gathered}
{ }^{\circ} I Z\left(\epsilon_{a}^{L}\right) M_{a^{*}, a}: V_{a^{*}} \otimes V_{a} \rightarrow \mathbb{C}, \\
{ }^{\circ} M_{a, a^{*}} Z\left({ }^{\circ} \epsilon_{a}^{L}\right) I: \mathbb{C} \rightarrow V_{a} \otimes V_{a^{*}} .
\end{gathered}
$$

Then we require that

this pairing is non-degenerate and can be used to identify $\left(V_{a}\right)^{*} \simeq V_{a^{*}}$.

Under this pairing, the product $M_{a, b}$ and the coproduct ${ }^{\circ} M_{b^{*}, a^{*}}$ are adjoint, etc. This again allows us to reduce the number of cases need to be mentioned below roughly by half.

Before proceeding, we note that we used $\epsilon_{a}^{L},{ }^{\circ} \epsilon_{a}^{R}$ to define the pairing. We can also use $\epsilon_{a^{*}}^{R},{ }^{\circ} \epsilon_{a^{*}}^{R}$ to define a slightly different pairing. Exactly which pairing to be used in each situation can be determined by fully assigning orientations to every line involved in the diagram. Below, we assume that every line carries an upward orientation, unless otherwise marked in the figure.

Second, a morphism can be moved across the product, see figure 43:

$$
M_{a, b}\left(Z(m) \otimes \operatorname{id}_{V_{b}}\right)=Z\left(m \otimes \operatorname{id}_{b}\right) M_{a, b} .
$$

Third, the map $I$ defined by the bowl geometry gives the unit, see figure 44 :

$$
M_{a, 1}(v \otimes I)=v, \quad v \in V_{a} .
$$




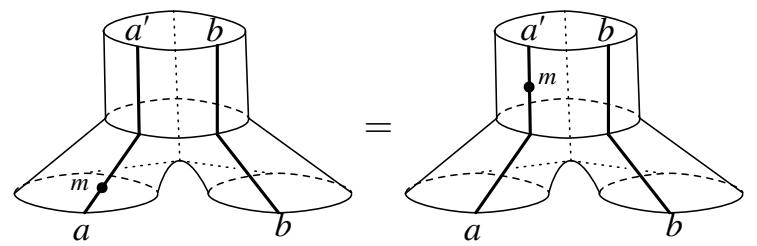

Figure 43. A morphism can be moved across the product.

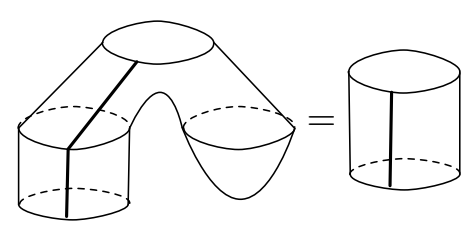

Figure 44. $I$ is a unit of the product $M_{a, 1}$.
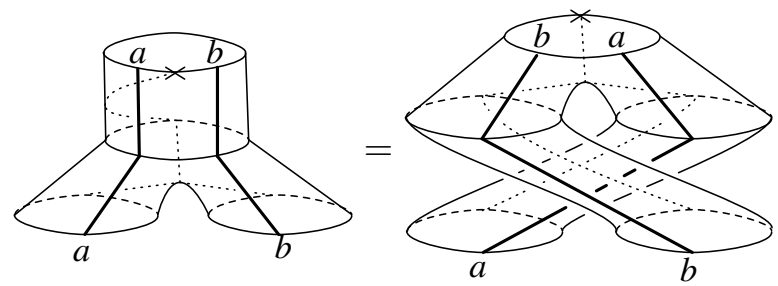

Figure 45. The product $M$ is twisted commutative.
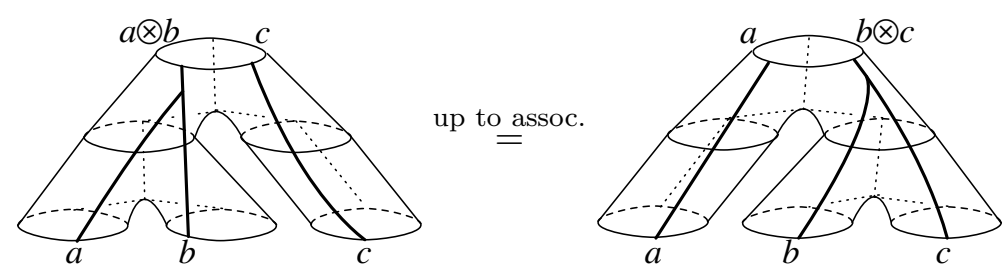

Figure 46. The product $M$ is associative up to the associator.

Fourth, it is twisted commutative:

$$
X_{a, b} M_{a, b}(v \otimes w)=M_{b, a}(w \otimes v), \quad v \in V_{a}, \quad w \in V_{b}
$$

as illustrated in figure 45. Fifth, it is associative up to the associator:

$$
Z\left(\alpha_{a, b, c}\right) M_{a \otimes b, c}\left(M_{a, b} \otimes \operatorname{id}_{c}\right)=M_{a, b \otimes c}\left(\operatorname{id}_{a} \otimes M_{b, c}\right),
$$

as shown in figure 46 .

Sixth, we want to formulate that the product is symmetric under the cyclic permutation of three circles. To do this we first introduce a slightly generalized form of the product shown in figure 47 :

$$
M_{\left(a \otimes c^{*}, c \otimes b^{*}\right) \rightarrow a \otimes b^{*}}: V_{a \otimes c^{*}} \otimes V_{c \otimes b^{*}} \rightarrow V_{a \otimes b^{*}}
$$




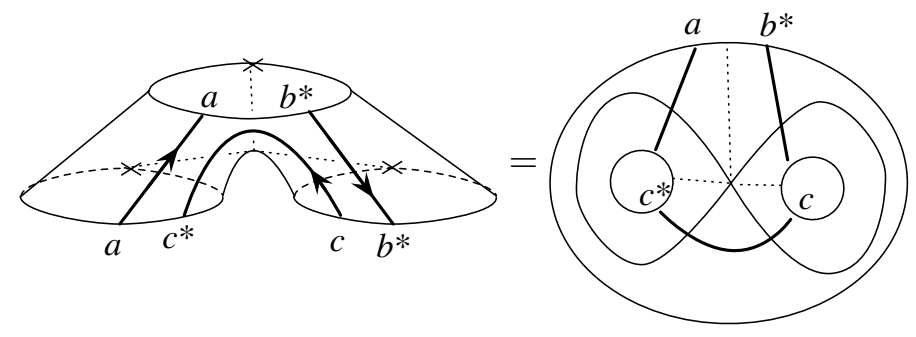

Figure 47. A slightly generalized version of the product operation. In the figure on the right, the time flows from inside to the outside.

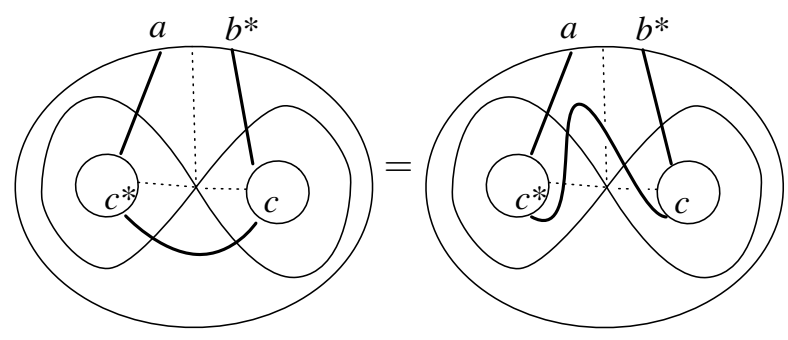

Figure 48. An alternative definition of the generalized product. The time flows from the inside to the outside.

given by

$$
M_{\left(a \otimes c^{*}, c \otimes b^{*}\right) \rightarrow a \otimes b^{*}}=Z\left(\operatorname{id}_{a} \otimes \epsilon_{c}^{L} \otimes \operatorname{id}_{b^{*}}\right) \mathcal{A}_{\left(a \otimes c^{*}\right) \otimes\left(c \otimes b^{*}\right) \rightarrow a \otimes\left(c^{*} \otimes c\right) \otimes b^{*}} M_{a \otimes c^{*}, c \otimes b^{*}}
$$

where $\epsilon_{c}^{L}: c^{*} \otimes c \rightarrow 1$ is the evaluation morphism and $\mathcal{A}$ is the generalized associator introduced in the last subsection.

The generalized product has an alternative definition as given in figure 48, where the line $c$ crosses three auxiliary lines. This gives an alternative expression

$$
\begin{aligned}
& M_{\left(a \otimes c^{*}, c \otimes b^{*}\right) \rightarrow a \otimes b^{*}}= \\
& \quad Z\left(\mathrm{id}_{a \otimes b^{*}} \otimes \epsilon_{c}^{R}\right) \mathcal{A}_{\left(c^{*} \otimes a\right) \otimes\left(b^{*} \otimes c\right) \rightarrow\left(a \otimes b^{*}\right) \otimes\left(c \otimes c^{*}\right)} M_{c^{*} \otimes a, b^{*} \otimes c}\left(X_{a, c^{*}} \otimes X_{c, b^{*}}\right)
\end{aligned}
$$

and we demand

$$
\text { the right hand sides of the equations (6.18) and (6.19) are the same. }
$$

We can now formulate the cyclic symmetry of the product:

$$
M_{\left(a \otimes c^{*}, c \otimes b^{*}\right) \rightarrow a \otimes b^{*}} \text { and } M_{\left(c \otimes b^{*}, b \otimes a^{*}\right) \rightarrow c \otimes a^{*}} \text { are related by the inner products, }
$$

see figure 49. We can in fact derive this relation for general $a, b, c$ just from the subcase when $c=1$ and the relations already mentioned. We keep the general case for cosmetic reasons, since it looks more symmetric.

Seventh, we need a consistency on the torus. An incoming circle consisting of four segments with lines $a, b, c, d$ can first split into two circles with two segments $a, b$ and 


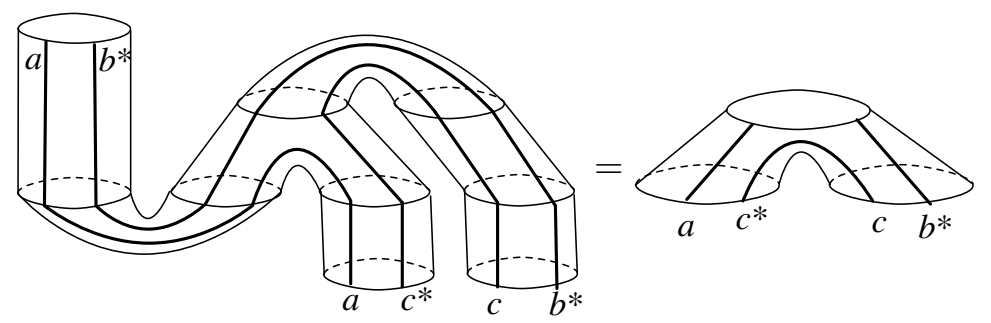

Figure 49. The product is invariant under exchanging an incoming circle and an outgoing circle.
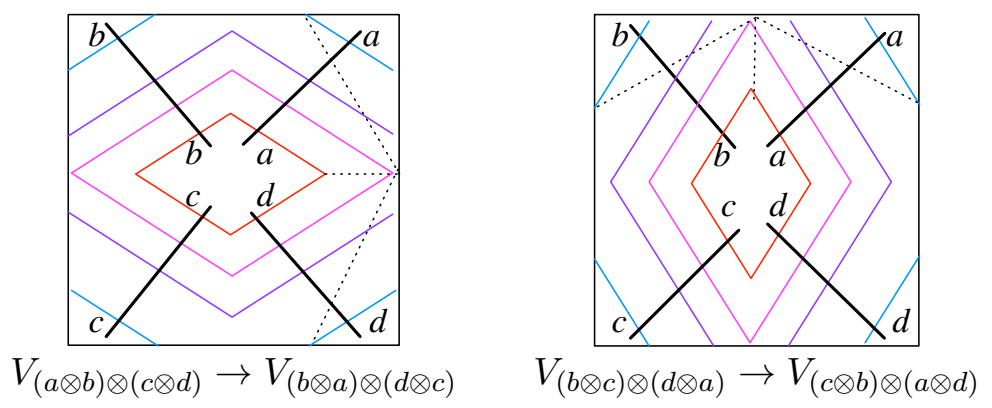

Figure 50. Two ways a circle splits into two and then rejoins. They should be equal up to the action of $X$ and the associators.

$c, d$ each and then rejoins to form a circle with four segments in the order $b, a, d, c$; another way this happens is that the two intermediate circles have segments $b, c$ and $d$, $a$, see figure 50. They each determine maps $(M \Delta)_{a, b ; c, d}: V_{(a \otimes b) \otimes(c \otimes d)} \rightarrow V_{(b \otimes a) \otimes(d \otimes c)}$ and $(M \Delta)_{b, c ; d, a}: V_{(b \otimes c) \otimes(d \otimes a)} \rightarrow V_{(c \otimes b) \otimes(a \otimes d)}$ given by

$$
\begin{aligned}
(M \Delta)_{a, b ; c, d} & :=M_{b \otimes a, d \otimes c}\left(X_{a, b} \otimes X_{c, d}\right) \Delta_{a \otimes b, c \otimes d}, \\
(M \Delta)_{b, c ; d, a} & :=M_{c \otimes b, a \otimes d}\left(X_{b, c} \otimes X_{d, a}\right) \Delta_{b \otimes c, d \otimes a} .
\end{aligned}
$$

We then demand that they are equal up to the generalized associators:

$$
\mathcal{A}_{(b \otimes a) \otimes(d \otimes c) \rightarrow(c \otimes b) \otimes(a \otimes d)}(M \Delta)_{a, b ; c, d}=(M \Delta)_{b, c ; d, a} \mathcal{A}_{(a \otimes b) \otimes(c \otimes d) \rightarrow(b \otimes c) \otimes(d \otimes a)} .
$$

Consistency in the general case. We finally finished writing down basic moves. Now we can analyze the general moves. We again have three cases:

- the change of the time function $t$ on the surface $\Sigma$,

- the change of the positions of the auxiliary line, and

- the change of the network in a disk region that does not change the morphism.

Let us start by discussing the third case. This is in fact automatic once the first two cases are taken care of, since any disk region can be put into a cylinder under a topological 


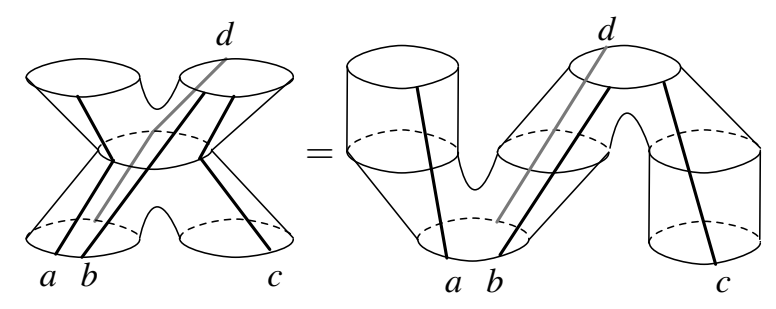

Figure 51. One possible topology change. The line $d$ is on the back side of the figures.

change, and then the auxiliary line can be moved off away from it. Then all we have to assume is that $Z(m)$ fuses appropriately, (6.3).

The change in the position of the auxiliary line can happen in the following three ways:

- The auxiliary line can move within a single cylinder. This was already discussed in the last subsection.

- When a circle with line $a$ and a circle with line $b$ join to form a circle, the order of $a, b$ and the base point $x$ in the product leg can either be $x, a, b$ or $a, b, x$. The invariance under this is the twisted commutativity (6.15).

- A line $c \in \mathcal{C}$ can cross the trivalent vertex of the auxiliary line. This move changes the number of the intersection of the line $c$ with the three auxiliary lines in one of the two ways, $0 \leftrightarrow 3$ or $1 \leftrightarrow 2$. One example of the move $0 \leftrightarrow 3$ is the equality (6.20) of the two definitions (6.18) and (6.19) of the generalized product. The move $1 \leftrightarrow 2$ can be deduced by combining the twisted commutativity.

Finally we need to take care of the changes in the time function $t$. One possible change is that a morphism and a product can happen in two different orders. The invariance under this move is (6.13). Then there are topological changes in the cutting of the surface, which again comes in the following varieties:

1. The creation or the annihilation of two critical points does figure 44. The consistency under this change is the unit property (6.14).

2a. The exchange of two critical points does figure 46. The consistency under this change is the associativity (6.16).

$2 \mathrm{~b}$. The number of intermediate circles changes from one to three. One example is drawn in figure 51. The consistency under this change can be reduced to the cyclic symmetry of the generalized product (6.21).

2c. How the torus is decomposed is changed as in figure 50, for which we assigned a basic relation (6.24).

Summarizing, a TFT with $\mathcal{C}$ symmetry is captured by the data $\left(V ; Z, X ;{ }^{\circ} I, I, M,{ }^{\circ} M\right)$ satisfying the various relations listed above. Namely, on the cylinder, we have (6.3), (6.5), (6.6), (6.7), (6.8), and on the general geometry, we have in addition (6.12), (6.13), (6.14), 
(6.15), (6.16), (6.20), (6.21), and (6.24), and finally, diagrams turned upside down correspond to adjoint linear maps.

\subsection{Gauged TFT with the dual symmetry}

Now we would like to discuss the definition of the TFT $T / A$ gauged by an algebra object $A$ in terms of the ungauged TFT $T$. We start from the data $\left(V ; Z, X ;{ }^{\circ} I, I, M,{ }^{\circ} M\right)$ for the original theory $T$.

The Hilbert space of the gauged theory was introduced in section 4.8.2. See that section for some necessary background. We use the action by $A$ on $V_{p}$, depicted in figure 24. This is given by

$$
P:=U_{p, A}\left({ }^{\circ} x_{R}, x_{L}\right):=Z\left(x_{L}\right) X_{p, A} Z\left({ }^{\circ} x_{R}\right)
$$

where $x_{L}: A \otimes p \rightarrow p$ and ${ }^{\circ} x_{R}: p \rightarrow p \otimes A$ are the morphisms defining the $A$-bimodule structure on $p$. As we already discussed, $P$ turns out to be a projector, and we define $W_{p}$ to be the projection $P V_{p}$. Now, we define the data $\left(W ; \tilde{Z}, \tilde{X},{ }^{\circ} \tilde{I}, \tilde{I}, \tilde{M},{ }^{\circ} \tilde{M}\right)$ for $T / A$ in terms of corresponding data for $T$.

The morphism map $\tilde{Z}$. We define the new $\tilde{Z}$ to be the restriction of the old $Z$. We need to check that if the initial state lay in $W_{p} \subset V_{p}$ then the final state also necessarily lies in $W_{q}$, for a bimodule morphism $p \rightarrow q$. This can be checked by gluing a cylinder on top of the final state which corresponds to the action of $A$. The wrapped $A$ line can then be taken across any bimodule morphism until it wraps the $p$ line at the start of the cobordism. But then the wrapped $A$ line has no effect and can be removed because the initial state we started with is invariant under the action of $A$.

The base point-change map $\tilde{\boldsymbol{X}}$. We now want to define the new $\tilde{X}_{p, q}: W_{p \otimes_{A} q} \rightarrow W_{q \otimes_{A} p}$. We use $\pi$ and ${ }^{\circ} \pi$ (see section 4.4) to define $\tilde{X}_{p, q}=Z(\pi) X_{p, q} Z\left({ }^{\circ} \pi\right)$. This is well defined because a wrapped $A$ line at the end of the cobordism can be moved to an $A$ line propagating between $a$ and $b$ at the start of the cobordism which can be removed because of the definition of $\otimes_{A}$.

The unit and counit maps $\tilde{\boldsymbol{I}}$ and ${ }^{\circ} \tilde{\boldsymbol{I}}$. Now the new unit morphism $\tilde{I}$ would be a map from $\mathbb{C}$ to $W_{A} \subset V_{A}$. We define it as $\tilde{I}=Z(u) I$ where $u: 1 \rightarrow A$ is the unit morphism in the definition of $A$. Similarly, $\tilde{\circ} I={ }^{\circ} I Z(v)$ where $v: A \rightarrow 1$ is the co-unit morphism in the definition of $A$. These are well-defined as can be shown by manipulations similar to those we are now going to perform for the definition of $\tilde{M}$.

The product and coproduct maps $\tilde{\boldsymbol{M}}$ and ${ }^{\circ} \tilde{\boldsymbol{M}}$. The new $\tilde{M}$ is defined analogously as $\tilde{M}_{p, q}=Z(\pi) M_{p, q}$. This $\tilde{M}$ can be shown to be well defined as a map $W_{p} \otimes W_{q} \rightarrow W_{p \otimes_{A} q}$ by a series of manipulations using a lot of properties of $A$. See figure 52 . We represent a pair of pants as a 3-punctured plane for ease of illustration. The lower punctures correspond to input legs and the upper puncture corresponds to the product leg of the pair of pants. Unlabeled lines correspond to $A$. To explain various manipulations, let us refer to manipulations involving $=_{i}$ as "step $i$ ". 

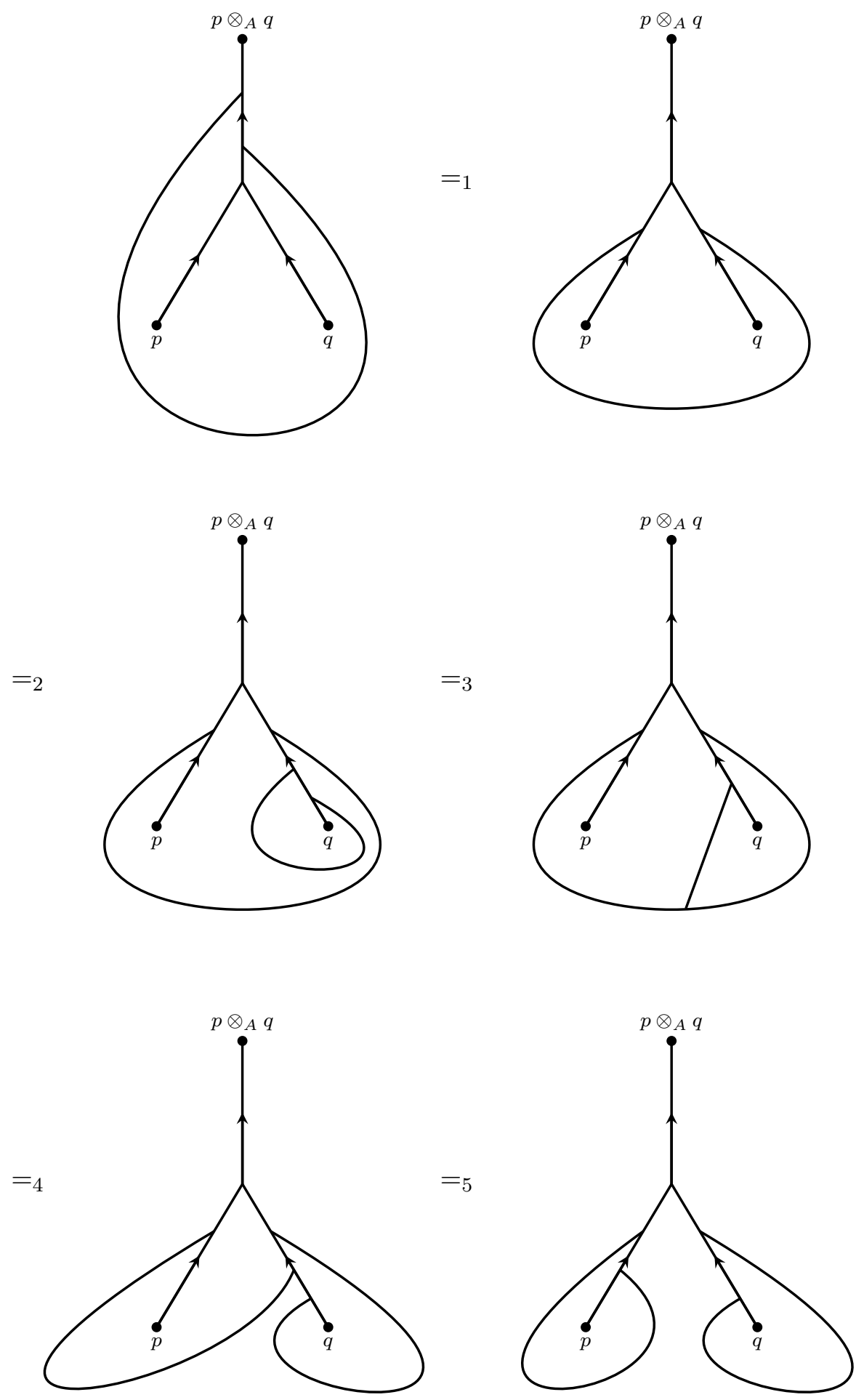

Figure 52. The new product $\tilde{M}$ and its well-definedness. For details of the manipulation, see the main text. 
- Step 1 just tells us that the left action of $A$ on $p \otimes_{A} q$ is defined by the left action on $p$, and the right action on $p \otimes_{A} q$ is defined by the right action on $q$.

- In step 2, we introduce an $A$ line wrapping the leg carrying $q$. We can do that because the input state in that leg is invariant under the action of $A$.

- In step 3 , we first use the fact that $q$ is an $A$-bimodule and then use the fact that $q$ is a right $A$-module.

- In step 4 , we use the fact that $q$ is a left module for $A$.

- Finally, in step 5, we first use the fact that $q$ is a bimodule, then the fact that the tensor product is $\otimes_{A}$, and then the fact that $p$ is a bimodule.

- Ultimately, we can simply remove both the $A$ lines because the input states are invariant under the action of $A$.

We define ${ }^{\circ} \tilde{M}$ as the adjoint of $\tilde{M}$.

To complete the definition of $T / A$, we have to check that the operations defined above satisfy the various conditions that we described in the last section. Most of them just concern some trivial topological manipulations of lines and are manifestly satisfied. Some others, such as (6.14), can be checked using manipulations similar to the ones we have been doing in this sub-section. Yet some others, like the complicated relation (6.20) and (6.24)), require us to simplify a lot of $\pi$ and ${ }^{\circ} \pi$, but this can be done. This completes the definition of $T / A$.

\section{Conclusions}

In this paper we reviewed the notion of unitary fusion categories, or symmetry categories as we prefer to call them, and how they formalize the generalized notion of finite symmetries of a two-dimensional system. We studied various explicit examples of such symmetry categories, some of which are related rather directly to finite groups and some of which are not. We then studied how a symmetry category can be gauged and be re-gauged back. We also defined $2 \mathrm{~d}$ topological quantum field theories admitting a symmetry given by a symmetry category. Many questions remain. Here we mention just two.

The first is how to generalize the constructions discussed in this paper to higher dimensions. In a sense, this is a merger of the generalized symmetry in two dimensions in the sense of this paper, and of the generalized symmetry in the sense of [2]. That there should be something that combines both is clear: even in general spacetime dimension $d$, the 0 -form symmetries can be any non-Abelian group $G$, possibly with an anomaly specified by $H^{d}(G, \mathrm{U}(1))$ in the bosonic case and by subtler objects in the fermionic case. Then the $(d-2)$-form symmetry needs to be extended at least to allow $\operatorname{Rep}(G)$. When $d=3$, it seems that the notion of 1 -form symmetries needs to be extended at least to include modular tensor categories, with an action of the 0 -form symmetry group $G$ with an anomaly. What should be the notion in $d=4$ and higher? 
The second is to actually construct two-dimensional systems $T$ for a given symmetry category $\mathcal{C}$. For any group $G$ without an anomaly, there is the trivial theory where the Hilbert space is always one-dimensional. How about the other cases? We can roughly classify symmetry categories $\mathcal{C}$ as follows, depending on the simplest possible theories $T$ that have $\mathcal{C}$ as a symmetry:

1. The simplest $\mathcal{C}$-symmetric theories have one-dimensional Hilbert space. These would be $\mathcal{C}$-symmetry protected topological (SPT) phases.

2. The simplest $\mathcal{C}$-symmetric theories have finite-dimensional Hilbert space. These would be $\mathcal{C}$-symmetry enriched topological (SET) phases.

3. The simplest $\mathcal{C}$-symmetric theories have infinite-dimensional Hilbert space. Taking the low energy limit, these would be $\mathcal{C}$-symmetric conformal field theories (CFTs).

4. There is no $\mathcal{C}$-symmetric theory.

Clearly this classification forms a hierarchy, and it would be nice if we have a uniform construction that tells easily which stage of the above classification a given symmetry category $\mathcal{C}$ belongs to. There is a recent paper in this general direction [36], where a construction of $2 \mathrm{~d}$ theory starting from any given symmetry category $\mathcal{C}$ was discussed. We hope to see more developments in the future.

Actually, there are various symmetry categories $\mathcal{C}$ constructed in the subfactor theory, e.g. what is called the Haagerup fusion category, for which no $\mathcal{C}$-symmetric theory is known. If a theory symmetric under the Haagerup fusion category could be constructed, it would be considered as a huge breakthrough.

\section{Acknowledgments}

The authors would like to thank Mikhail Kapranov for bringing the crucial reference [7] to the authors' attention. LB is also grateful to Kevin Costello, Davide Gaiotto and Theo Johnson-Freyd for helpful discussions. The authors thank Nils Carqueville and Eric Sharpe for comments on, and Marcel Bischoff, Kentaro Hori and Shu-Heng Shao for pointing out erroneous claims in an earlier version of the manuscript. The work of LB is partially supported by the Perimeter Institute for Theoretical Physics. Research at Perimeter Institute is supported by the Government of Canada through Industry Canada and by the Province of Ontario through the Ministry of Economic Development and Innovation. The work of YT is partially supported in part by JSPS KAKENHI Grant-in-Aid (Wakate-A), No.17H04837 and JSPS KAKENHI Grant-in-Aid (Kiban-S), No.16H06335 and by WPI Initiative, MEXT, Japan at IPMU, the University of Tokyo. 


\section{A Group cohomology}

In this appendix, we collect various standard facts about group cohomology.

Definition. Given a finite group $G$ and its module $A$, we define $n$-cochains $C^{n}(G, A)$ as functions $G^{n} \rightarrow A$. The differential is given by

$$
\begin{aligned}
d f\left(g_{1}, \ldots, g_{n+1}\right)= & g_{1} f\left(g_{2}, \ldots, g_{n+1}\right) \\
& +\sum_{i=1}^{n}(-1)^{i} f\left(g_{1}, \ldots, g_{i} g_{i+1}, \ldots, g_{n+1}\right)+(-1)^{n+1} f\left(g_{1}, \ldots, g_{n}\right) .
\end{aligned}
$$

The differential squares to zero: $d^{2}=0$. Then we define the group cohomology $H^{i}(G, A)$ as the cohomology of this differential. Explicitly, the first few differentials are given by

$$
\begin{aligned}
d f(g, h) & =g f(h)-f(g h)+f(g), \\
d f(a, b, c) & =a f(b, c)-f(a b, c)+f(a, b c)-f(a, b), \\
d f(x, y, z, w) & =x f(y, z, w)-f(x y, z, w)+f(x, y z, w)-f(x, y, z w)+f(x, y, z) .
\end{aligned}
$$

Some points on notation. It does not lead to any loss of generality if we assume that every cochain/cocycle/coboundary is normalized, i.e. it is zero whenever at least one of $g_{i}=1$. See e.g. [13]. We have assumed throughout the paper that every cochain is normalized. We are often interested in $H^{i}(G, \mathrm{U}(1))$ for $i=2,3$ where the action of $G$ on $\mathrm{U}(1)$ is taken to be trivial. Henceforth, we will assume the trivial action whenever we write $\mathrm{U}(1)$. It is also convenient to treat $\mathrm{U}(1)$ elements as phases and in this case the + sign in above definitions should be replaced by the usual multiplication of phases. For instance we have,

$$
d f(g, h)=\frac{f(h) f(g)}{f(g h)} .
$$

We have used the product notation throughout the paper in the context of group cohomology valued in $\mathrm{U}(1)$.

Pull-back. Recall that given a map $M_{1} \rightarrow M_{2}$ between two manifolds, one can pull-back $n$-forms on $M_{2}$ to $n$-forms on $M_{1}$. The analogous statement in group cohomology is that given a group homomorphism $G \rightarrow G^{\prime}$, we obtain a module homomorphism $H^{i}\left(G^{\prime}, A\right) \rightarrow$ $H^{i}(G, A)$. Explicitly, let $h: G \rightarrow G^{\prime}$, then $\tilde{h}: H^{i}\left(G^{\prime}, A\right) \rightarrow H^{i}(G, A)$ is given by

$$
\tilde{h}(\alpha)\left(g_{1}, \cdots, g_{i}\right)=\alpha\left(h\left(g_{1}\right), \cdots, h\left(g_{i}\right)\right) .
$$

Cup product. There is an operation called cup product $C^{i}(G, A) \times C^{j}(G, A) \rightarrow C^{i+j}(G, A)$ when $A$ is a ring. If $\alpha \in H^{i}(G, A)$ and $\beta \in H^{j}(G, A)$, then the cup product is defined as

$$
(\alpha \cup \beta)\left(g_{1}, \cdots, g_{i+j}\right)=\alpha\left(g_{1}, \cdots, g_{i}\right) \beta\left(g_{i+1}, \cdots, g_{i+j}\right) .
$$

It can be easily checked that this product descends to a product on cohomologies. 
One-dimensional representations of $G$. Let us ask what is the meaning of $H^{1}(G, \mathrm{U}(1))$. The 1-cochains are maps from $G$ to $\mathrm{U}(1)$ and imposing the cocycle condition turns them into group homomorphisms. Hence $H^{1}(G, \mathrm{U}(1))$ is the group formed by one-dimensional representations of $G$. In particular, when $G$ is a finite Abelian group, then $H^{1}(G, \mathrm{U}(1)) \simeq \hat{G}$ is the dual group.

Projective representations of $\boldsymbol{G}$. Now, let us ask what is the meaning of $\epsilon \in H^{2}(G, \mathrm{U}(1))$. We want to interpret the $\epsilon\left(g_{1}, g_{2}\right)$ as the phases defining a projective representation of $G$. The cocyle condition reads

$$
\epsilon\left(g_{1}, g_{2}\right) \epsilon\left(g_{1} g_{2}, g_{3}\right)=\epsilon\left(g_{2}, g_{3}\right) \epsilon\left(g_{1}, g_{2} g_{3}\right)
$$

which is the associativity condition such phases are required to satisfy. Such a cocycle can be shifted by a coboundary of the form

$$
d \beta\left(g_{1}, g_{2}\right)=\frac{\beta\left(g_{1} g_{2}\right)}{\beta\left(g_{1}\right) \beta\left(g_{2}\right)}
$$

which corresponds to rephasing of the group action on the projective representation. Thus, we see that $H^{2}(G, \mathrm{U}(1))$ classifies the phases encountered in projective representations upto rephasing. The usual representations correspond to the trivial element of $H^{2}(G, \mathrm{U}(1))$.

Crossed products and extensions of groups. Consider an Abelian group $H$ and a (possibly non-Abelian) group $K$. Consider an action of $K$ on $H$ and use it to define $H^{2}(K, H)$. An element $\kappa \in H^{2}(K, H)$ can be used to define a group extension $G$ of $K$ by $H$, that is there is a short exact sequence

$$
0 \rightarrow H \rightarrow G \rightarrow K \rightarrow 0
$$

and $G$ is called the $\kappa$-cross product of $K$ and $H$ and it is written as $G=H \rtimes_{\kappa} K$. Explicitly, the group $G$ as a set is the direct product $H \times K$ with the group multiplication given as follows

$$
\left(h_{1}, k_{1}\right)\left(h_{2}, k_{2}\right)=\left(h_{1}+\left(k_{1} \triangleright h_{2}\right)+\kappa\left(k_{1}, k_{2}\right), k_{1} k_{2}\right) .
$$

Here, $\triangleright$ denotes the action of $K$ on $H$ via inner automorphism in $G$. The reader can verify that the associativity of the group multiplication is ensured by the cocycle condition on $\kappa$. Shifting $\kappa$ by a coboundary changes $G$ upto isomorphism. Hence, group extensions of $K$ by an Abelian group $H$ are classified by a group action of $K$ on $H$ along with an element in $H^{2}(K, H)$ defined using the group action.

Bicharacters on $G$. Consider an Abelian group $G$ and a trivial module $A$ of $G$. Given a cohomology element in $H^{2}(G, A)$ represented by a cocycle $\epsilon(g, h)$, one can form $\alpha(g, h)=$ $\epsilon(g, h)-\epsilon(h, g)$ which is an antisymmetric function on $G$. This is indeed well defined because adding a coboundary to $\epsilon$ doesn't change $\alpha$. When $A$ is $\mathrm{U}(1)$ this $\alpha$ is a bicharacter, and there's a bijection between an antisymmetric bicharacter on $G$ and $H^{2}(G, \mathrm{U}(1))$. 
A useful isomorphism. Recall the familiar statement that any closed $n$-form is exact locally. In group cohomology, the analogous statement is that

$$
H^{i}(G, \mathbb{R})=1,
$$

that is $H^{i}(G, \mathbb{R})$ is trivial. We can use this to obtain $H^{i+1}(G, \mathbb{Z}) \simeq H^{i}(G, \mathrm{U}(1))$. This follows from the long exact sequence associated to the sequence $0 \rightarrow \mathbb{Z} \rightarrow \mathbb{R} \rightarrow \mathrm{U}(1) \rightarrow 0$.

\section{Explicit group cohomologies.}

- $H^{*}\left(\mathbb{Z}_{n}, \mathbb{Z}\right)=\mathbb{Z}\left[x_{2}\right] /\left(n x_{2}\right)$.

- $H^{2}\left(\left(\mathbb{Z}_{n}\right)^{k}, \mathrm{U}(1)\right)=\left(\mathbb{Z}_{n}\right)^{k(k-1) / 2}$.

- $H^{3}\left(\left(\mathbb{Z}_{n}\right)^{k}, \mathrm{U}(1)\right)=\left(\mathbb{Z}_{n}\right)^{k+k(k-1) / 2+k(k-1)(k-2) / 6}$, with generators given by

$$
\begin{aligned}
\alpha^{(i)}(a, b, c) & =e^{2 \pi i a_{i}\left(b_{i}+c_{i}-\left\langle b_{i}+c_{i}\right\rangle\right) / n^{2}}, \\
\alpha^{(i, j)}(a, b, c) & =e^{2 \pi i a_{i}\left(b_{j}+c_{j}-\left\langle b_{j}+c_{j}\right\rangle\right) / n^{2}}, \\
\alpha^{(i, j, k)}(a, b, c) & =e^{2 \pi i a_{i} b_{j} c_{k} / n}
\end{aligned}
$$

where $a, b, c=\{0,1, \ldots, n-1\}$ and $\langle a\rangle$ is the $\bmod n$ function to $\{0, \ldots, n-1\}$. In particular, $H^{3}\left(\mathbb{Z}_{n}, \mathrm{U}(1)\right)=\mathbb{Z}_{n}$ and its generator has the cocycle

$$
\alpha(a, b, c)=e^{2 \pi i a(b+c-\langle b+c\rangle) / n^{2}} .
$$

- For $D_{m}$ the dihedral group with $m$ elements we have [37],

$$
H^{*}\left(D_{m}, \mathbb{Z}\right)=\mathbb{Z}\left[a_{2}, b_{2}, c_{3}, d_{4}\right] /\left(2 a_{2}, 2 b_{2}, 2 c_{3}, m d_{4},\left(b_{2}\right)^{2}+a_{2} b_{2}+\left(m^{2} / 4\right) d_{4}\right) .
$$

In particular, $H^{2}\left(D_{2 n+1}, \mathrm{U}(1)\right)=0, H^{3}\left(D_{2 n+1}, \mathrm{U}(1)\right)=\mathbb{Z}_{4 n+2} ; H^{2}\left(D_{2 n}, \mathrm{U}(1)\right)=$ $\mathbb{Z}_{2} \times \mathbb{Z}_{2}, H^{3}\left(D_{2 n}, \mathrm{U}(1)\right)=\mathbb{Z}_{2} \times \mathbb{Z}_{2} \times \mathbb{Z}_{2 n}$. The explicit generators can be found in [38].

- For $Q_{8}$, the quaternion group, we have [39]

$$
H^{*}\left(Q_{8}, \mathbb{Z}\right)=\mathbb{Z}\left[A_{2}, B_{2}, C_{4}\right] /\left(2 A_{2}, 2 B_{2}, 8 C_{4}, A_{2}^{2}, B_{2}^{2}, A_{2} B_{2}-4 C_{4}\right) .
$$

Open Access. This article is distributed under the terms of the Creative Commons Attribution License (CC-BY 4.0), which permits any use, distribution and reproduction in any medium, provided the original author(s) and source are credited.

\section{References}

[1] C. Vafa, Quantum Symmetries of String Vacua, Mod. Phys. Lett. A 4 (1989) 1615 [inSPIRE].

[2] D. Gaiotto, A. Kapustin, N. Seiberg and B. Willett, Generalized Global Symmetries, JHEP 02 (2015) 172 [arXiv: 1412.5148] [INSPIRE].

[3] I. Brunner, N. Carqueville and D. Plencner, Discrete torsion defects, Commun. Math. Phys. 337 (2015) 429 [arXiv:1404.7497] [INSPIRE]. 
[4] E. Sharpe, Notes on generalized global symmetries in QFT, Fortsch. Phys. 63 (2015) 659 [arXiv: 1508.04770] [INSPIRE].

[5] D. Gaiotto, A. Kapustin, Z. Komargodski and N. Seiberg, Theta, Time Reversal and Temperature, JHEP 05 (2017) 091 [arXiv:1703.00501] [INSPIRE].

[6] M. Bischoff, R. Longo, Y. Kawahigashi and K.-H. Rehren, Tensor categories of endomorphisms and inclusions of von Neumann algebras, arXiv:1407.4793 [INSPIRE].

[7] P. Etingof, S. Gelaki, D. Nikshych and V. Ostrik, Tensor categories, Mathematical Surveys and Monographs, vol. 205, AMS, Providence, RI (2015).

[8] N. Carqueville and I. Runkel, Orbifold completion of defect bicategories, Quantum Topol. 7 (2016) 203 [arXiv:1210.6363] [INSPIRE].

[9] I. Brunner, N. Carqueville and D. Plencner, A quick guide to defect orbifolds, Proc. Symp. Pure Math. 88 (2014) 231 [arXiv:1310.0062] [INSPIRE].

[10] G.W. Moore and G. Segal, D-branes and k-theory in 2D topological field theory, hep-th/0609042 [INSPIRE].

[11] G.W. Moore and N. Seiberg, Lectures on RCFT, in Strings '89, Proceedings of the Trieste Spring School on Superstrings, World Scientific (1990)

[http://www.physics.rutgers.edu/ gmoore/LecturesRCFT.pdf] [INSPIRE].

[12] R. Dijkgraaf and E. Witten, Topological Gauge Theories and Group Cohomology, Commun. Math. Phys. 129 (1990) 393 [inSPIRE].

[13] X. Chen, Z.-C. Gu, Z.-X. Liu and X.-G. Wen, Symmetry protected topological orders and the group cohomology of their symmetry group, Phys. Rev. B 87 (2013) 155114 [arXiv: 1106.4772] [INSPIRE].

[14] M.R. Douglas, D-branes and discrete torsion, hep-th/9807235 [INSPIRE].

[15] D. Tambara and S. Yamagami, Tensor categories with fusion rules of self-duality for finite abelian groups, J. Algebra 209 (1998) 692.

[16] P. Etingof and S. Gelaki, Isocategorical groups, Int. Math. Res. Not. (2001) 59, [math/0007196].

[17] M. Izumi and H. Kosaki, On a subfactor analogue of the second cohomology, Rev. Math. Phys. 14 (2002) 733.

[18] J. Fuchs, I. Runkel and C. Schweigert, TFT construction of RCFT correlators 1. Partition functions, Nucl. Phys. B 646 (2002) 353 [hep-th/0204148] [INSPIRE].

[19] G. Schaumann, Traces on module categories over fusion categories, J. Algebra 379 (2013) 382 [arXiv: 1206.5716$]$.

[20] P. Etingof, D. Nikshych and V. Ostrik, Fusion categories and homotopy theory, Quantum Topol. 1 (2010) 209 [arXiv:0909.3140].

[21] L. Bhardwaj, D. Gaiotto and A. Kapustin, State sum constructions of spin-TFTs and string net constructions of fermionic phases of matter, JHEP 04 (2017) 096 [arXiv:1605.01640] [INSPIRE].

[22] L. Kong, Anyon condensation and tensor categories, Nucl. Phys. B 886 (2014) 436 [arXiv: 1307.8244$]$ [INSPIRE]. 
[23] I. Brunner, N. Carqueville and D. Plencner, Orbifolds and topological defects, Commun. Math. Phys. 332 (2014) 669 [arXiv:1307.3141] [INSPIRE].

[24] G.W. Moore and N. Seiberg, Classical and Quantum Conformal Field Theory, Commun. Math. Phys. 123 (1989) 177 [inSPIRE].

[25] V. Ostrik, Fusion categories of rank 2, Math. Res. Lett. 10 (2003) 177 [math/0203255].

[26] V. Ostrik, Pivotal fusion categories of rank 3, Mosc. Math. J. 15 (2015) 373 [arXiv:1309.4822].

[27] J. Fröhlich, J. Fuchs, I. Runkel and C. Schweigert, Defect lines, dualities and generalised orbifolds, in Proceedings, 16th International Congress on Mathematical Physics (ICMP09), Prague, Czech Republic, August 3-8, 2009 [DOI:10.1142/9789814304634_0056] [arXiv: 0909.5013] [INSPIRE].

[28] V. Ostrik, Module categories, weak Hopf algebras and modular invariants, Transform. Groups 8 (2003) 177 [math/0111139].

[29] D. Naidu, Categorical Morita equivalence for group-theoretical categories, Comm. Algebra 35 (2007) 3544 [math/0605530].

[30] D. Naidu and D. Nikshych, Lagrangian subcategories and braided tensor equivalences of twisted quantum doubles of finite groups, Commun. Math. Phys. 279 (2008) 845 [arXiv: 0705.0665].

[31] B. Uribe, On the classification of pointed fusion categories up to weak morita equivalence, arXiv: 1511.05522.

[32] P. Etingof, S. Gelaki and V. Ostrik, Classification of fusion categories of dimension pq, Int. Math. Res. Not. 57 (2004) 3041 [math/0304194].

[33] H.I. Blau, Fusion rings with few degrees, J. Algebra 396 (2013) 220.

[34] G.I. Kac and V.G. Paljutkin, Finite ring groups, Trans. Moscow Math. Soc. (1966) 251 [Tr. Mosk. Mat. Obs. 15 (1966) 224].

[35] E. Meir and E. Musicantov, Module categories over graded fusion categories, J. Pure Appl. Algebra 216 (2012) 2449 [arXiv:1010.4333].

[36] M. Buican and A. Gromov, Anyonic Chains, Topological Defects and Conformal Field Theory, Commun. Math. Phys. 356 (2017) 1017 [arXiv:1701.02800] [InSPIRE].

[37] D. Handel, On products in the cohomology of the dihedral groups, Tohoku Math. J. (2) 45 (1993) 13.

[38] M.D.F. de Wild Propitius, Topological interactions in broken gauge theories, Ph.D. Thesis, University of Amsterdam (1995) [hep-th/9511195] [INSPIRE].

[39] T. Hayami and K. Sanada, Cohomology ring of the generalized quaternion group with coefficients in an order, Commun. Algebra 30 (2002) 3611. 